Supporting Information

\title{
ATRP Initiators Based on Proton Transfer Benzazole Dyes: Solid State Photoactive Polymer with Very Large Stokes Shift
}

Jônatas Faleiro Berbigier, ${ }^{a}$ Luís Gustavo Teixeira Alves Duarte, ${ }^{b}$ Matheus Fialho Zawacki, ${ }^{c}$ Bruno Bercini de Araújo, ${ }^{d}$ Cristtofer de Moura Santos, ${ }^{d}$ Teresa

Dib Zambon Atvars, ${ }^{b}$ Paulo Fernando Bruno Gonçalves, ${ }^{d}$ César Liberato Petzhold, ${ }^{c}$ Fabiano Severo Rodembusch, ${ }^{\text {a* }}$

aGrupo de Pesquisa em Fotoquímica Orgânica Aplicada, Universidade Federal do Rio Grande do Sul - Instituto de Química, Av. Bento Gonçalves 9500. CEP 91501-970. Porto Alegre, RS, Brazil. ${ }^{b}$ Chemistry Institute, Physical Chemistry Department, University of Campinas, Av. Sergio Buarque de Holanda s/n. CEP 13083-970. Campinas, SP, Brazil.

'Laboratório de Síntese Orgânica e Polímeros, Chemistry Institute, Universidade Federal do Rio Grande do Sul (UFRGS), Av. Bento Gonçalves, 9500, Porto Alegre, RS, Brazil.

¿Grupo de Química Teórica. Instituto de Química. Universidade Federal do Rio Grande do Sul. Av. Bento Gonçalves 9500. CEP 91501-970. Porto Alegre, RS, Brazil.

E-mail: rodembusch@iq.ufrgs.br* 
Spectroscopic characterization

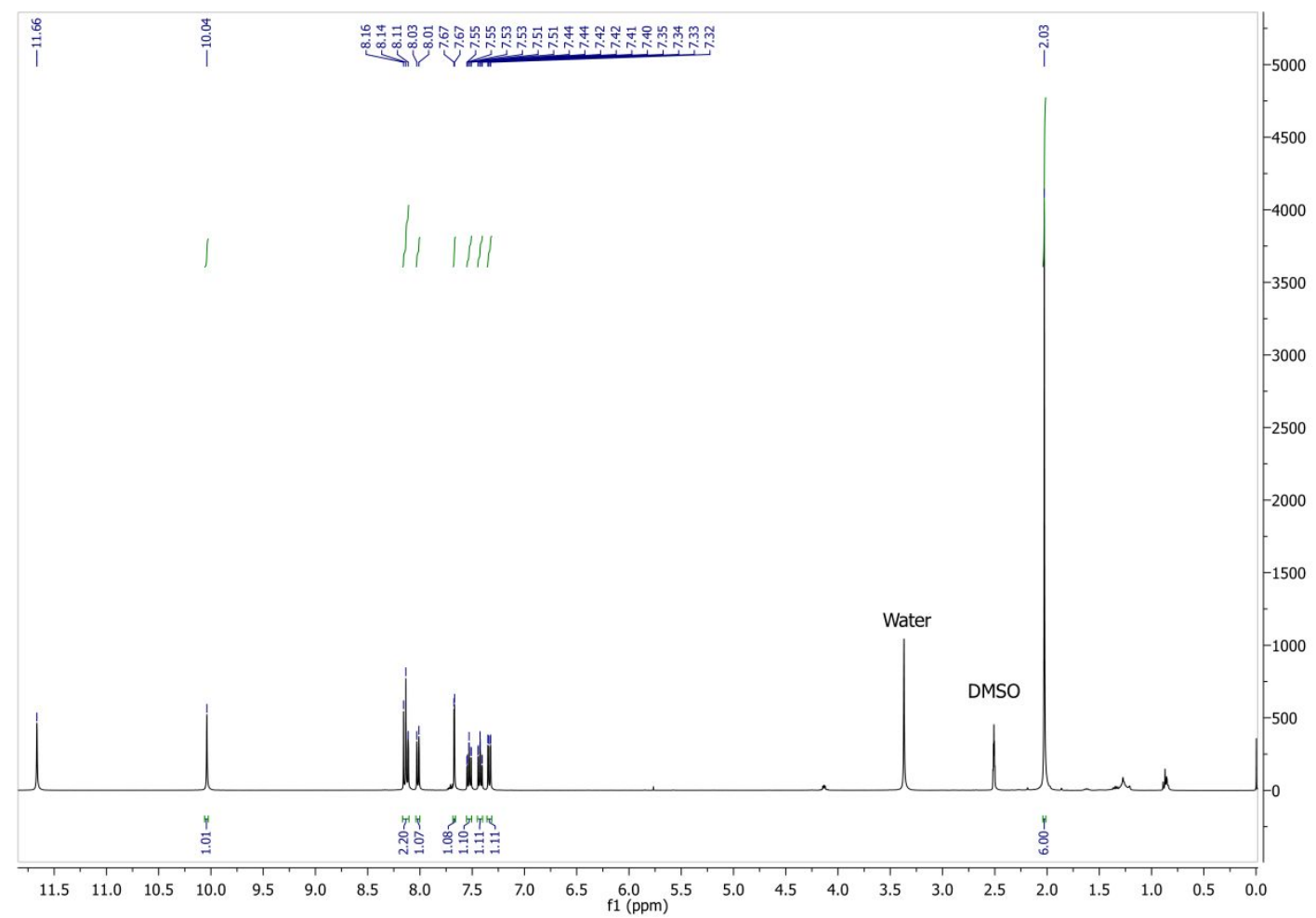

Figure S1. ${ }^{1} \mathrm{H}-\mathrm{NMR}$ spectrum of 6.

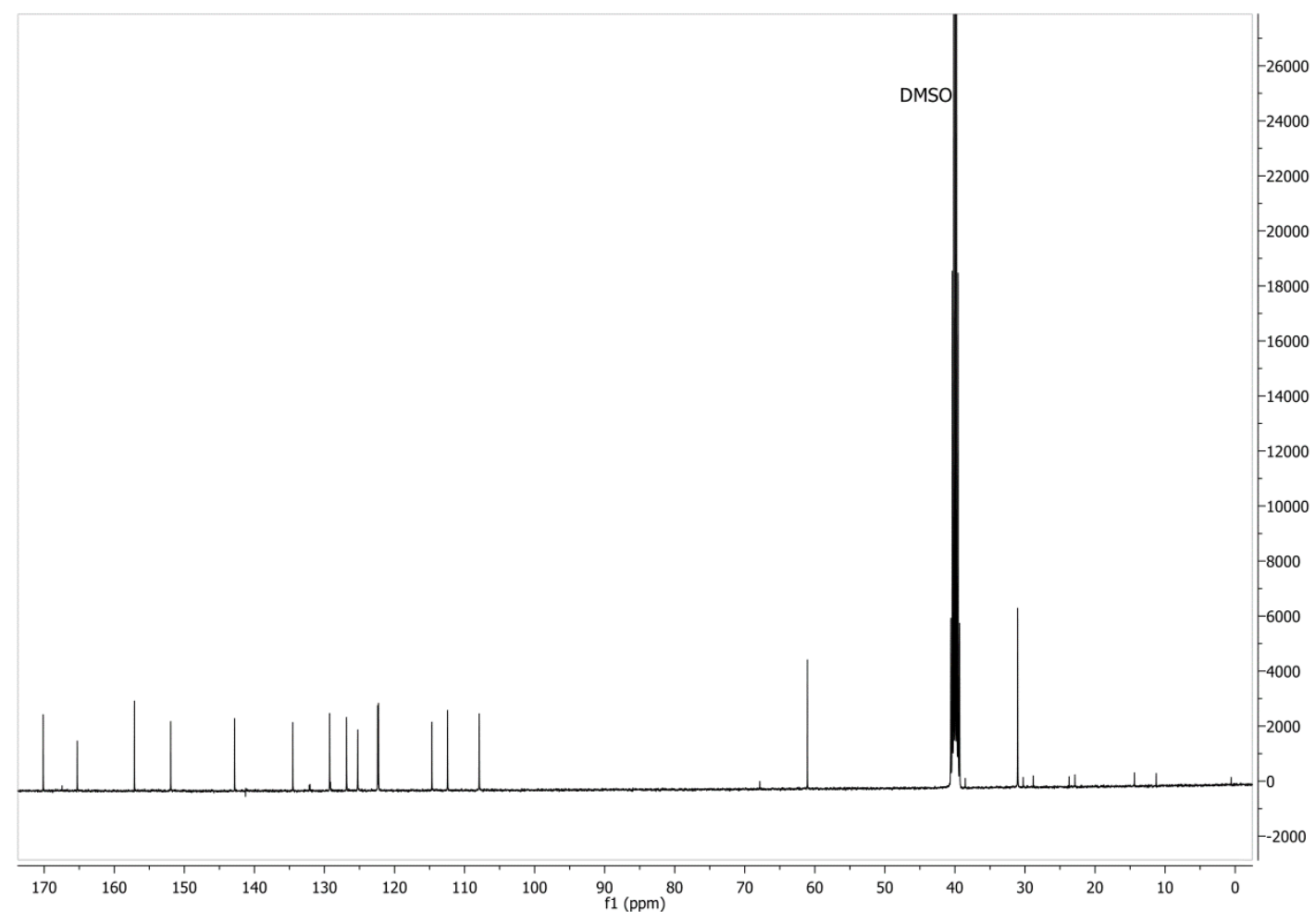

Figure S2. ${ }^{13} \mathrm{C}-\mathrm{NMR}$ spectrum of 6 . 


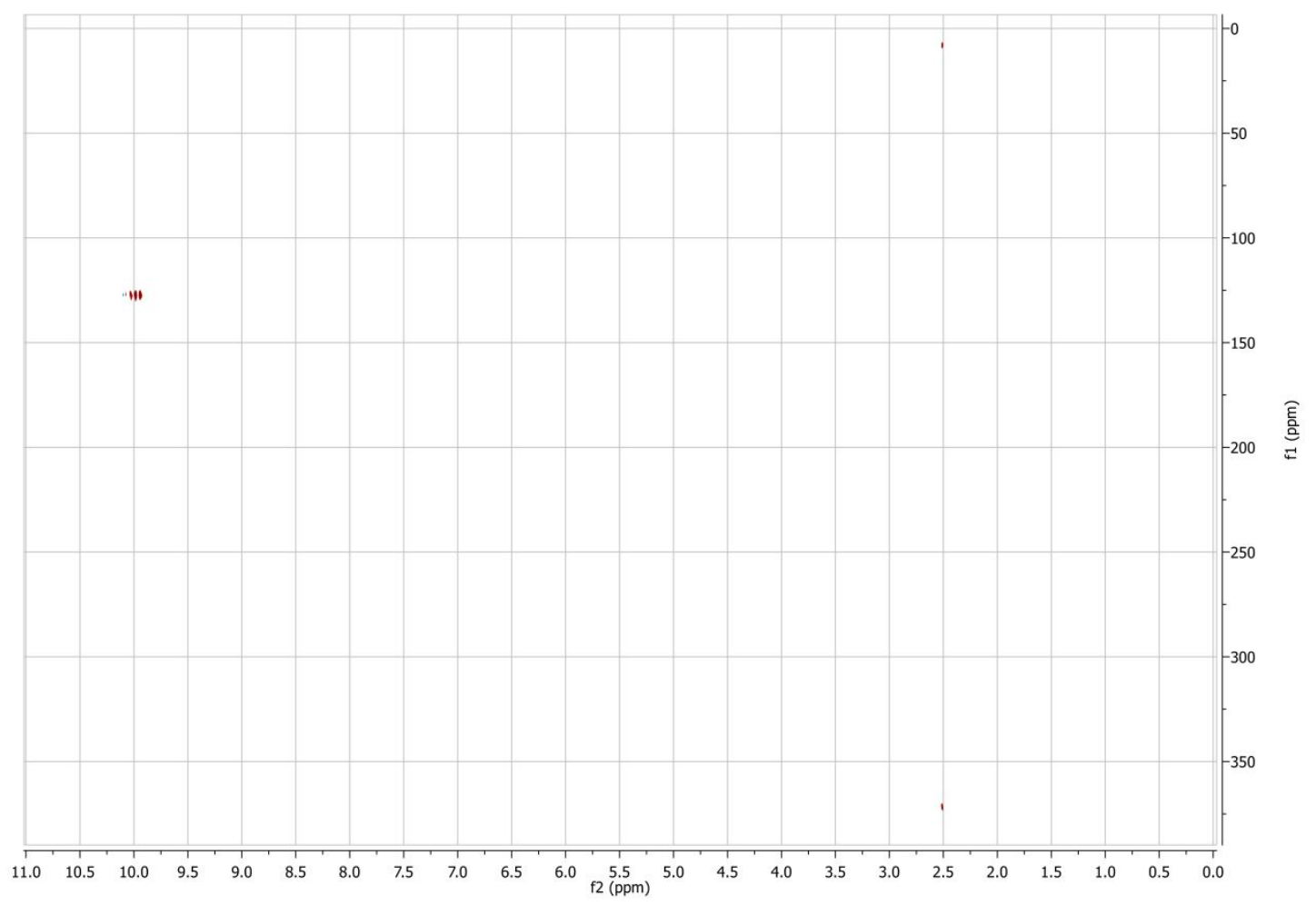

Figure S3. ${ }^{1} \mathrm{H}-{ }^{14} \mathrm{~N}-\mathrm{HMQC}$ spectrum of 6.

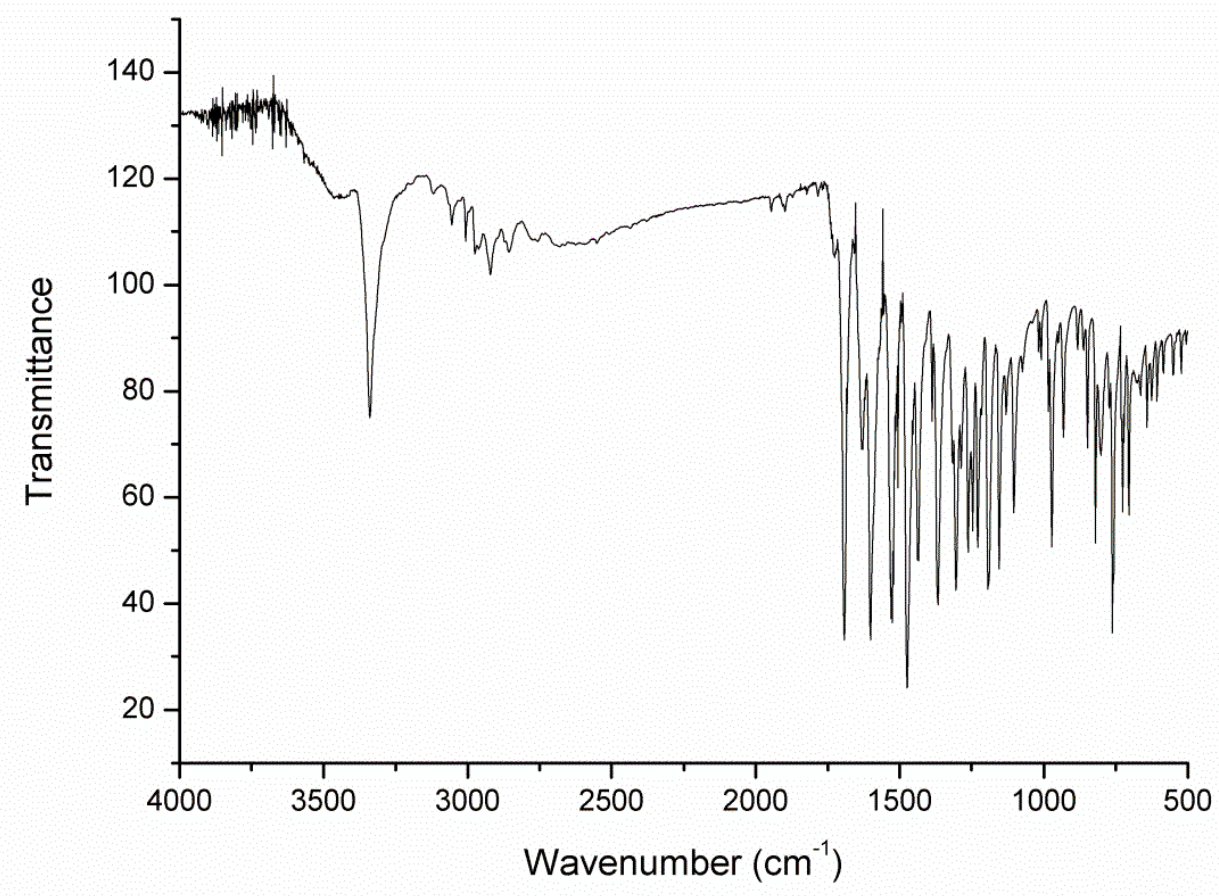

Figure S4. FTIR spectra of 6. 


\begin{tabular}{|c|c|c|c|c|c|c|c|}
\hline Meas. $\mathrm{m} / \mathrm{z}$ & lon Formula & $\mathrm{m} / \mathrm{z}$ & $\begin{array}{c}\text { err } \\
{[\mathrm{ppm}]}\end{array}$ & mSigma & rdb & $\mathrm{e}^{-}$Conf & N-Rule \\
\hline 391,0118 & C17H16BrN2O2S & 391,0110 & -1.8 & 15.1 & 10.5 & even & ok \\
\hline
\end{tabular}

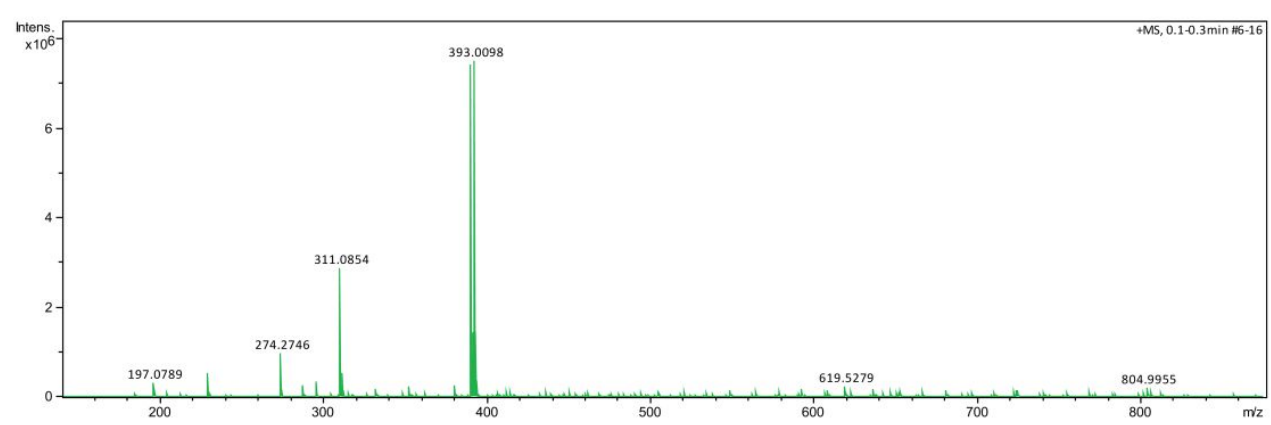

Figure S5. UHPLC-QTOF-MS of 6.

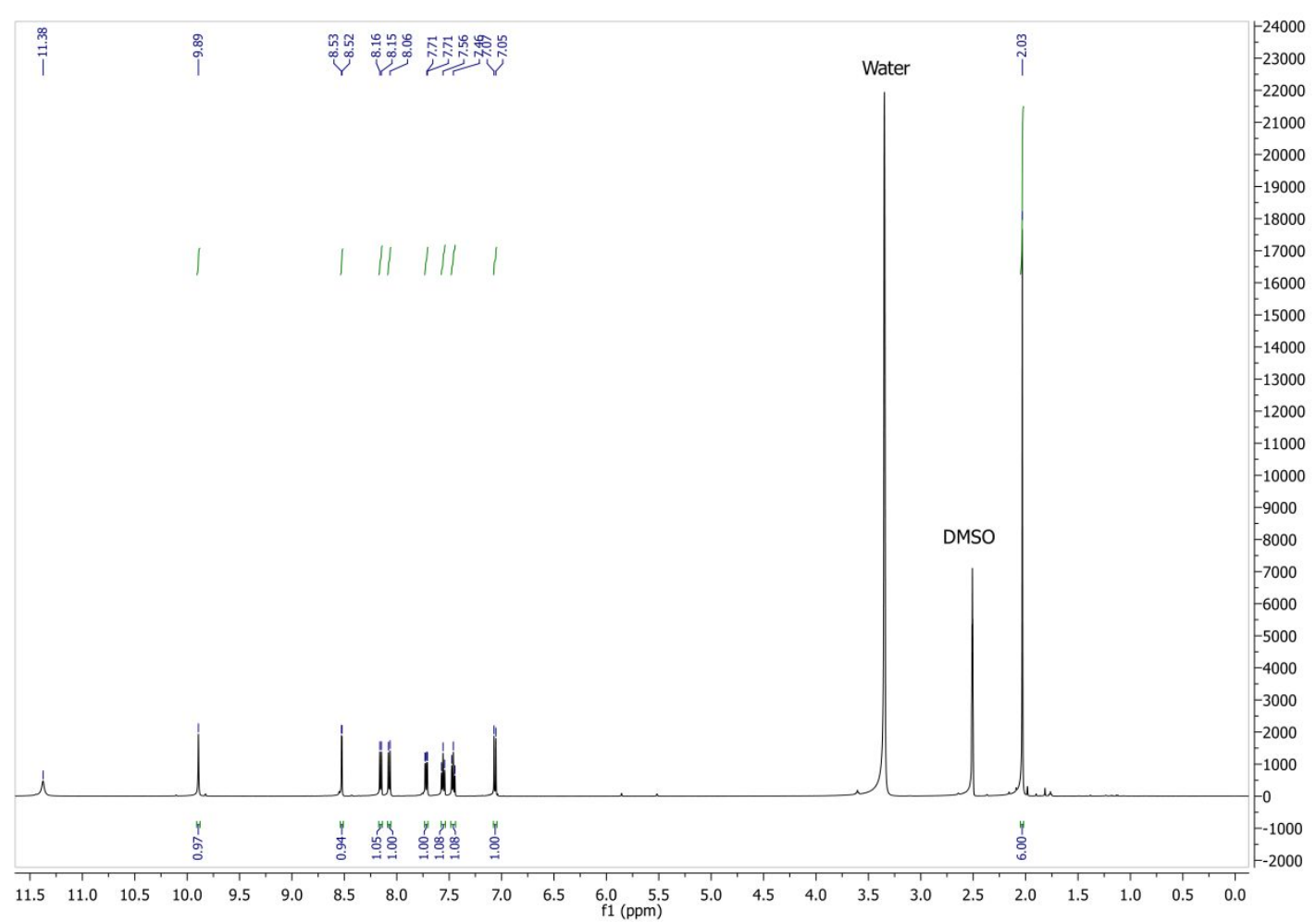

Figure S6. ${ }^{1} \mathrm{H}-\mathrm{NMR}$ spectrum of 7. 


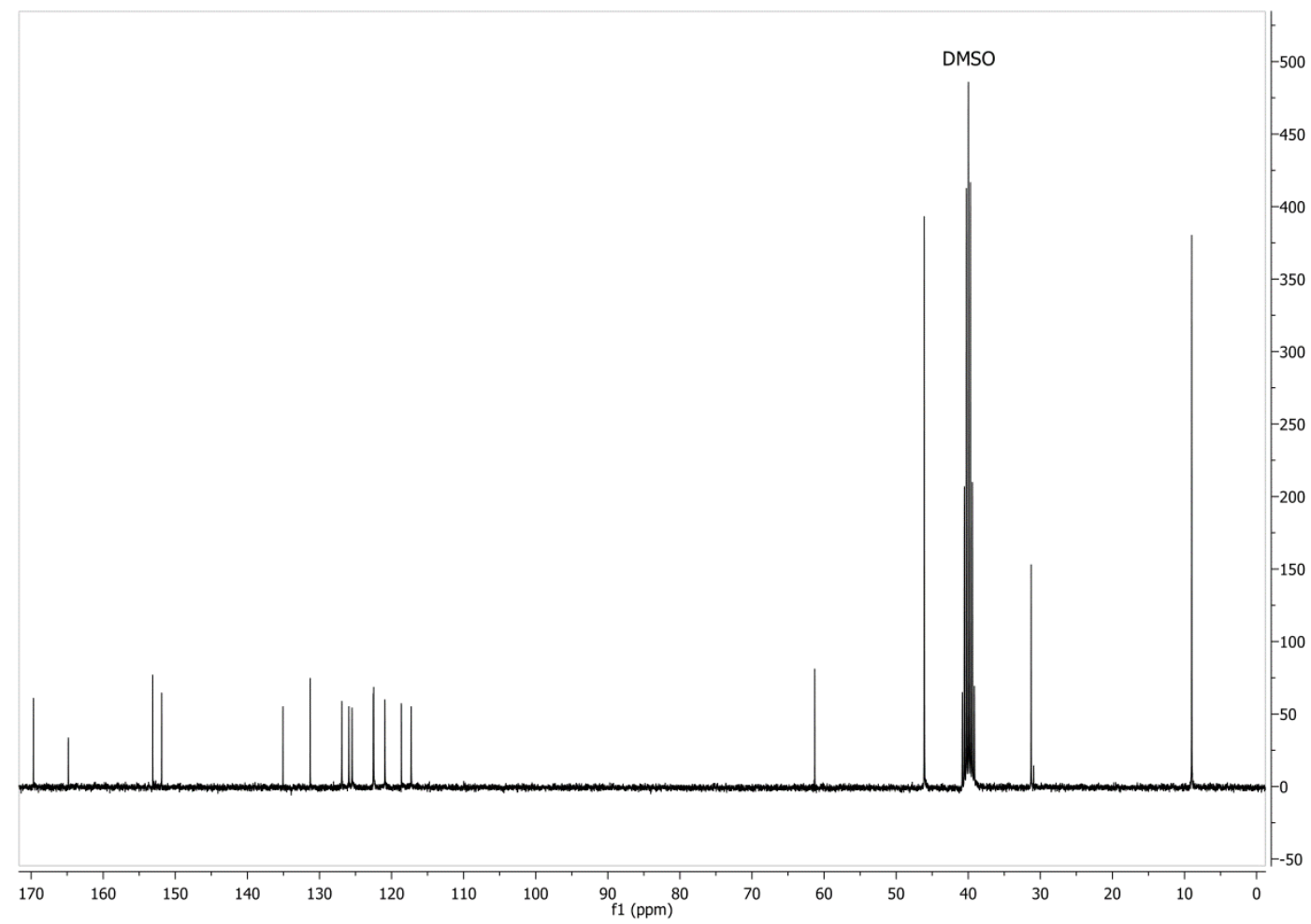

Figure S7. ${ }^{13} \mathrm{C}-\mathrm{NMR}$ spectrum of 7.

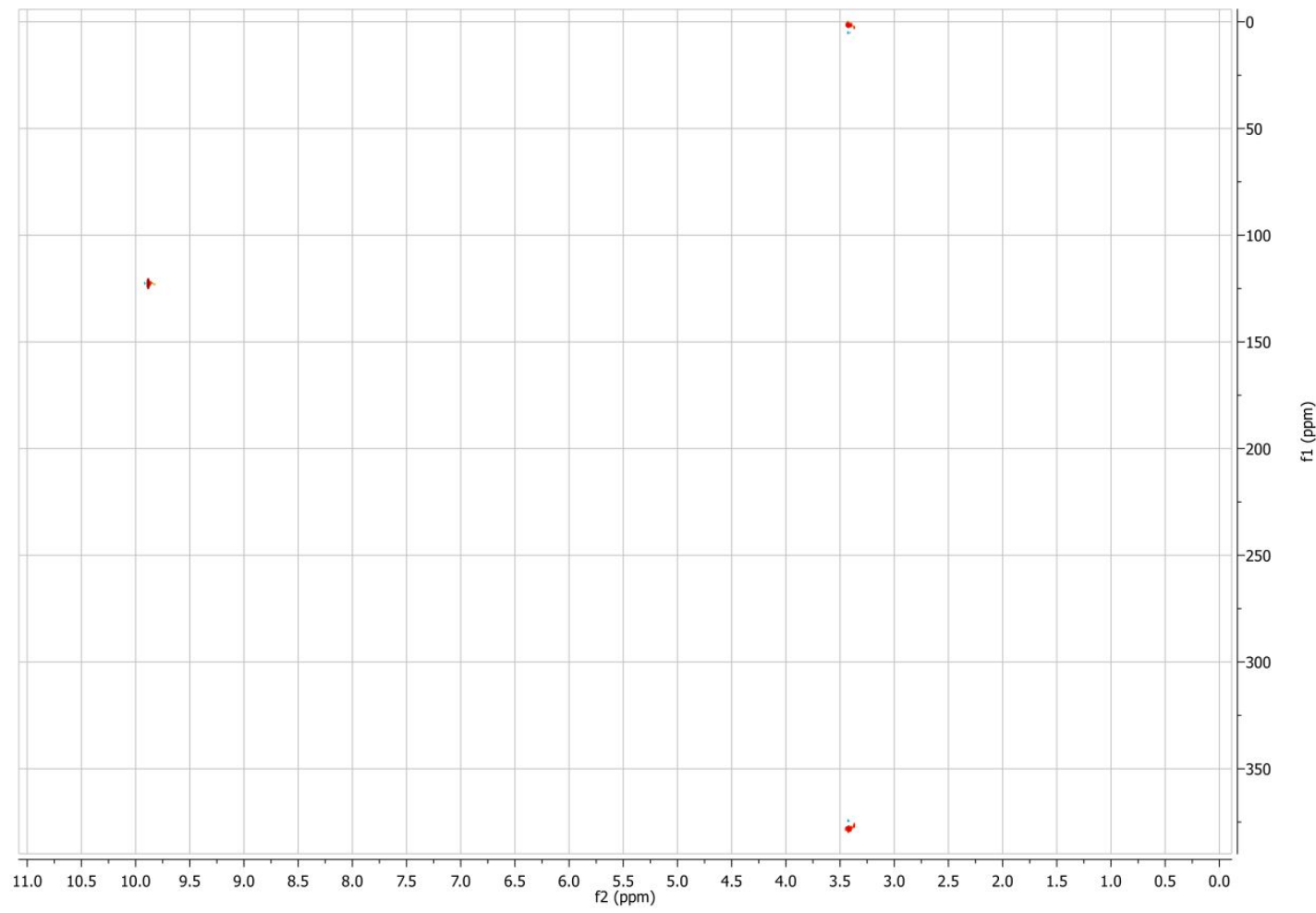

Figure S8. ${ }^{1} \mathrm{H}-{ }^{14} \mathrm{~N}-\mathrm{HMQC}$ spectrum of 7. 


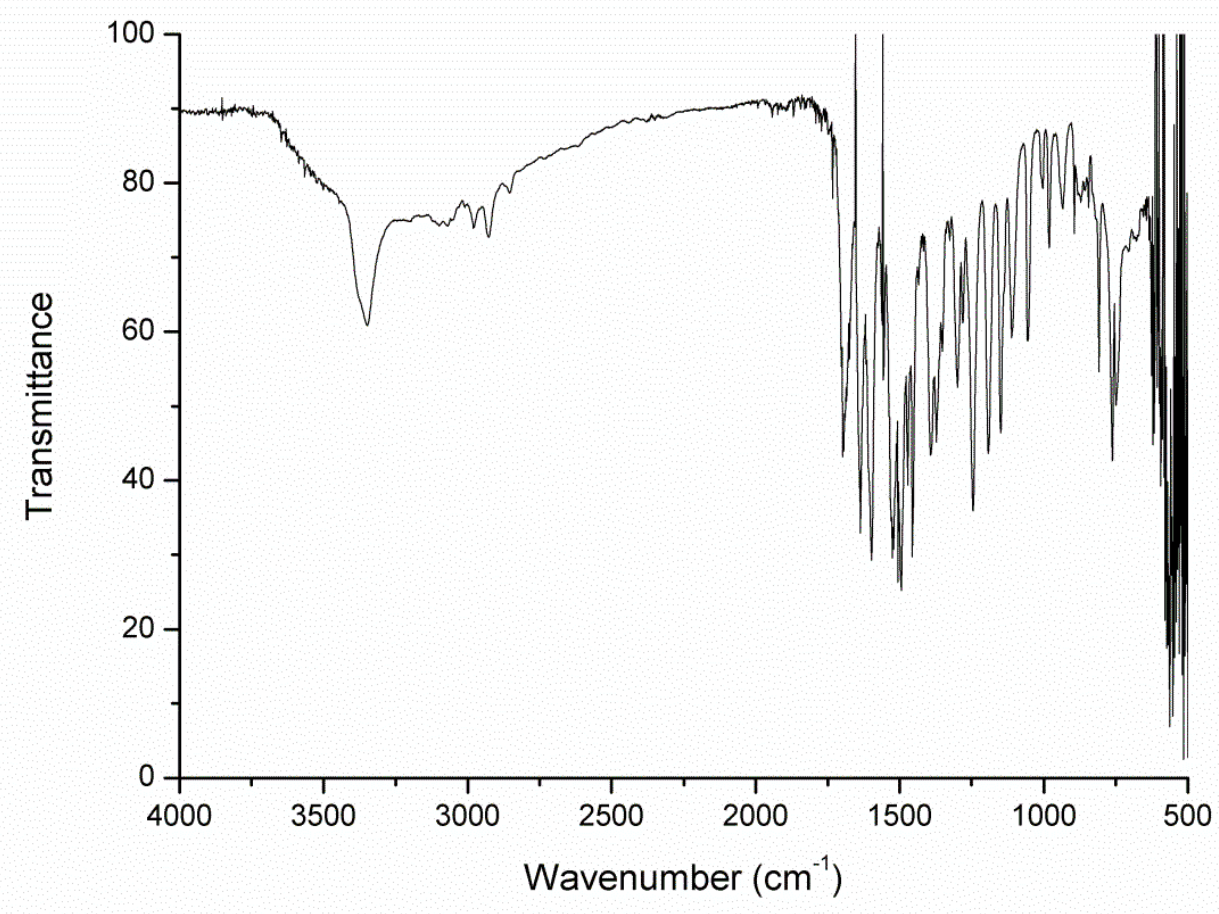

Figure S9. FTIR spectrum of 7.

\begin{tabular}{|c|c|c|c|c|c|c|c|}
\hline Meas. $\mathrm{m} / \mathrm{z}$ & lon Formula & $\mathrm{m} / \mathbf{z}$ & $\begin{array}{c}\text { err } \\
{[\mathrm{ppm}]}\end{array}$ & $\mathrm{mSigma}$ & $\mathrm{rdb}$ & $\mathrm{e}^{-}$Conf & N-Rule \\
\hline 391,0110 & C17H16BrN2O2S & 391,0110 & -0.0 & 22.4 & 10.5 & even & ok \\
\hline
\end{tabular}

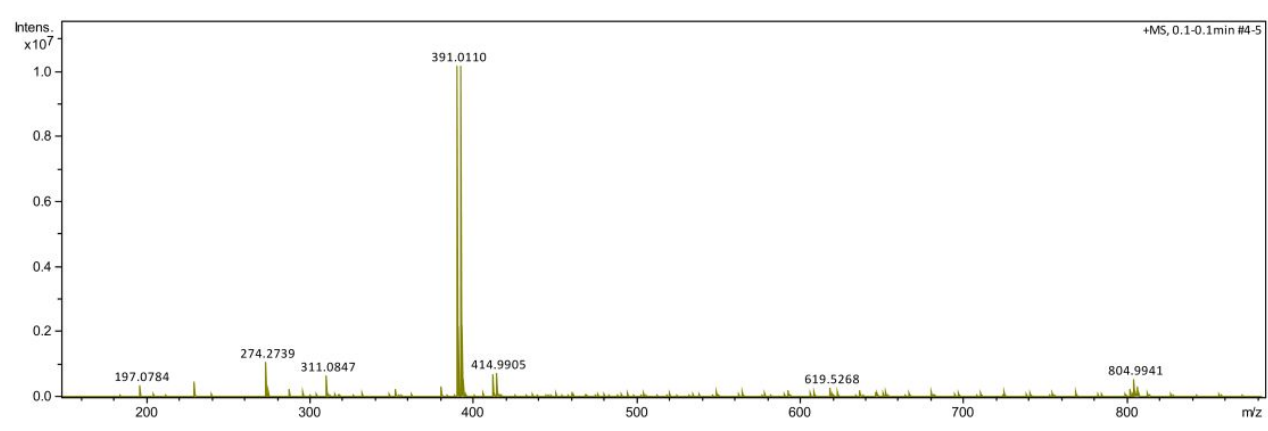

Figure S10. UHPLC-QTOF-MS of 7. 


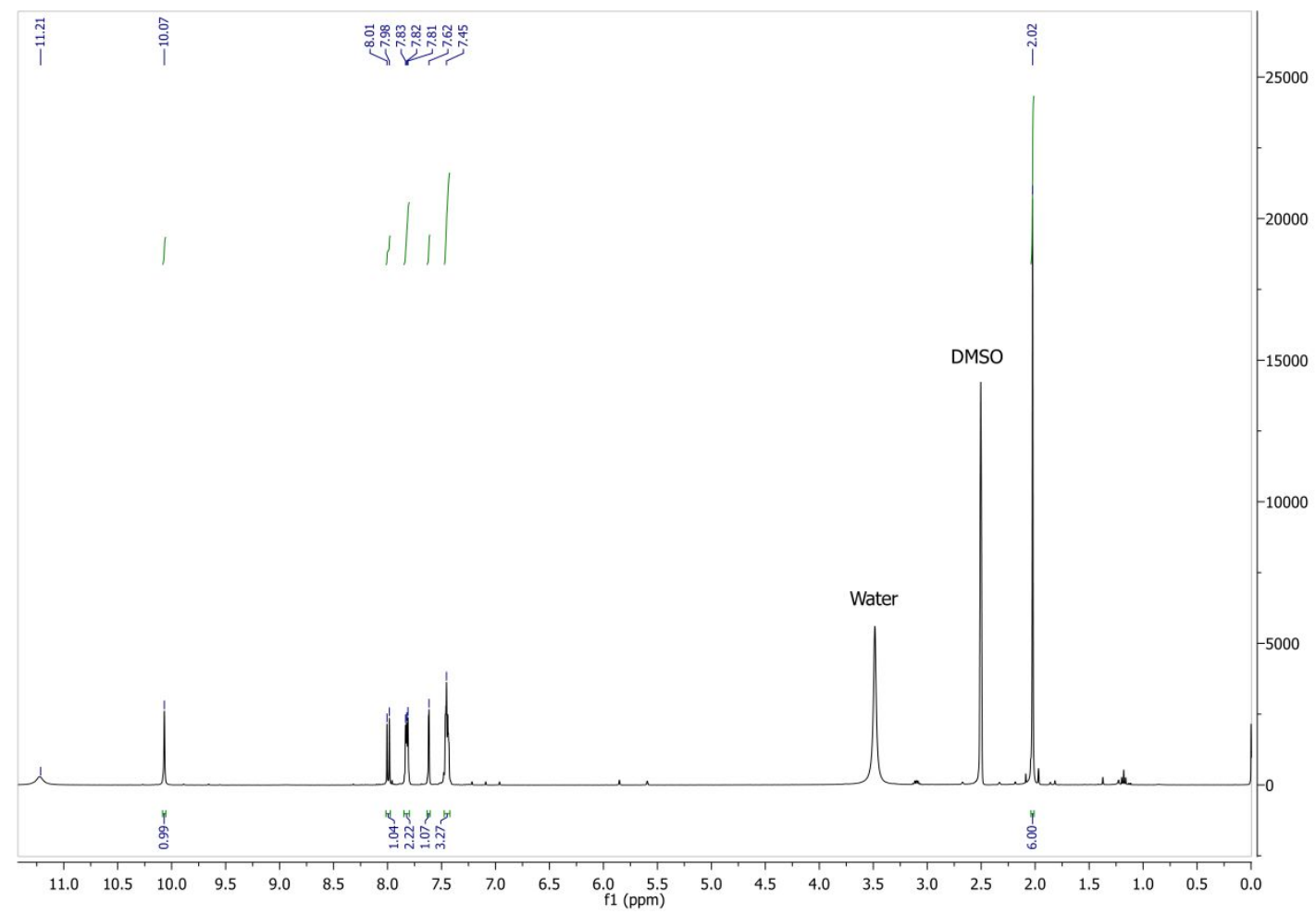

Figure S11. ${ }^{1} \mathrm{H}-\mathrm{NMR}$ spectrum of 8.

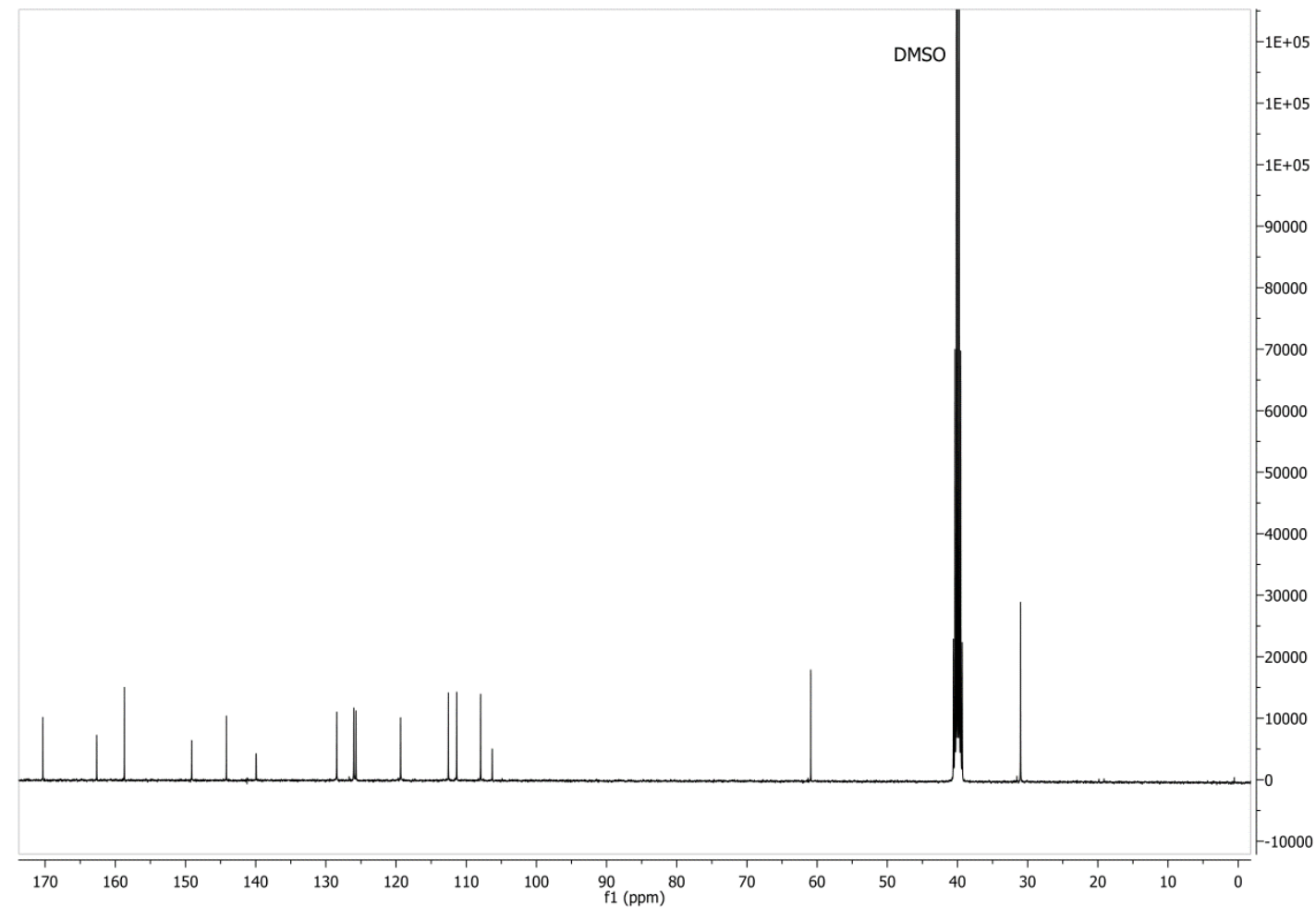

Figure S12. ${ }^{13} \mathrm{C}-\mathrm{NMR}$ spectrum of 8. 


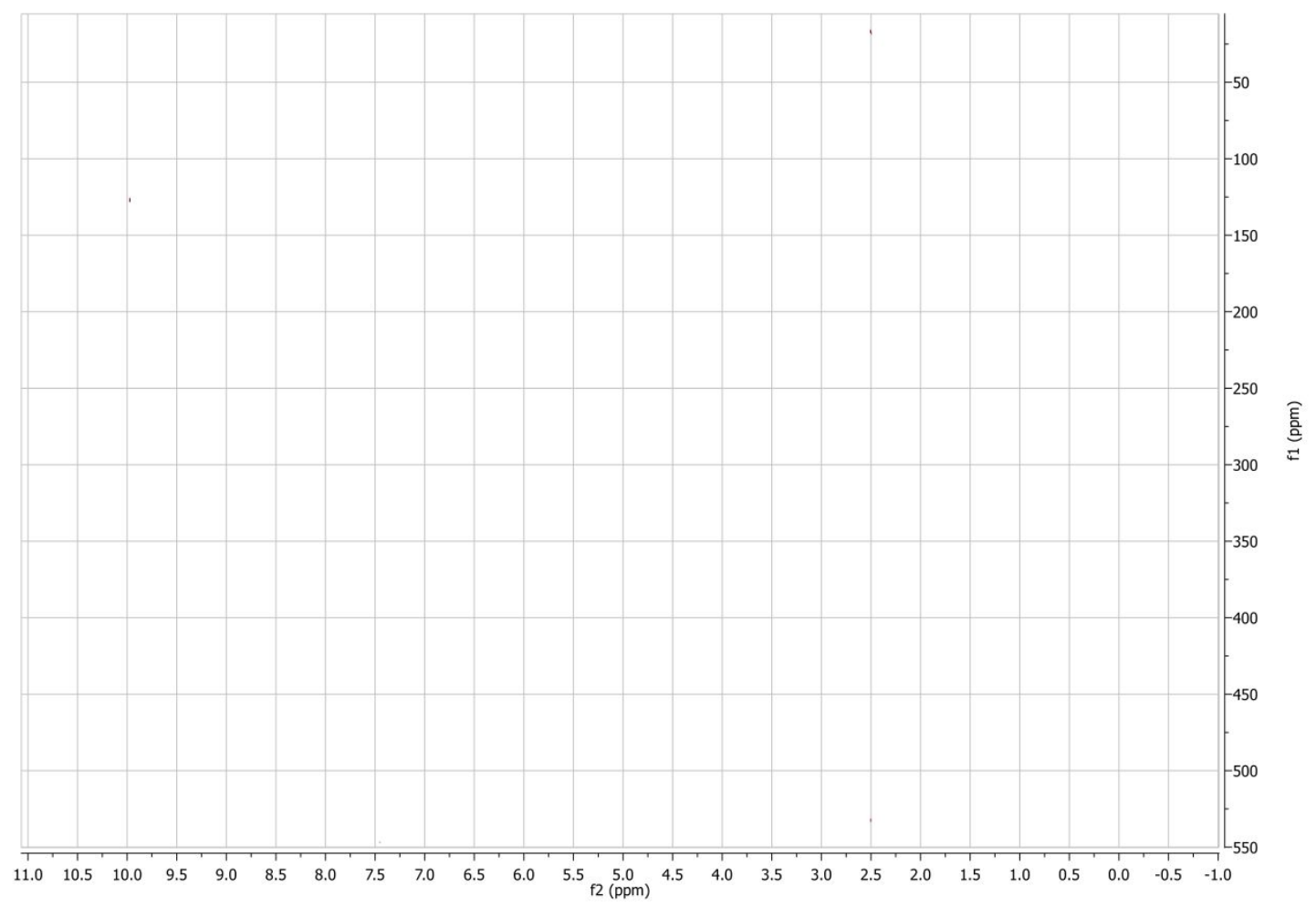

Figure S13. ${ }^{1} \mathrm{H}-{ }^{14} \mathrm{~N}-\mathrm{HMQC}$ spectrum of 8.

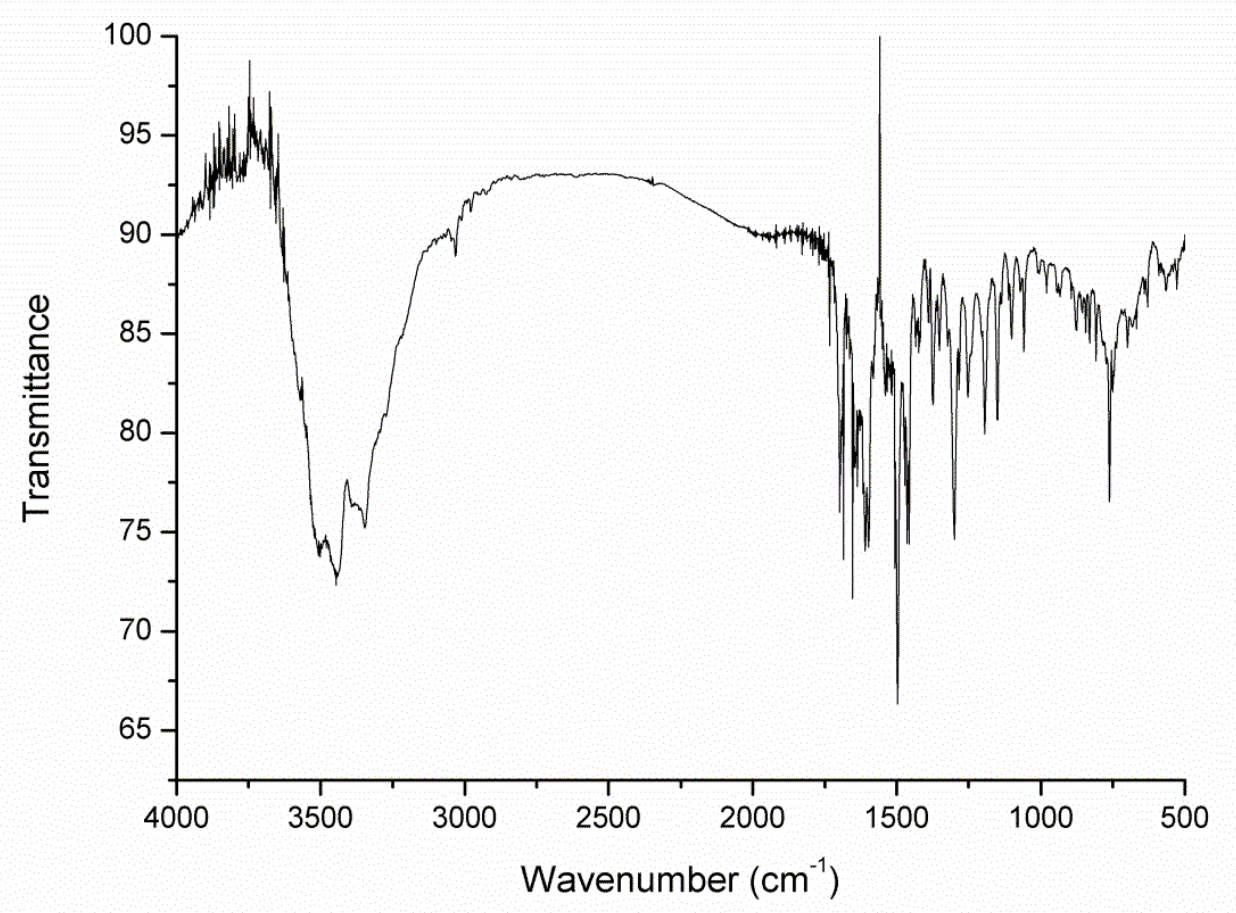

Figure S14. FTIR spectrum of 8. 


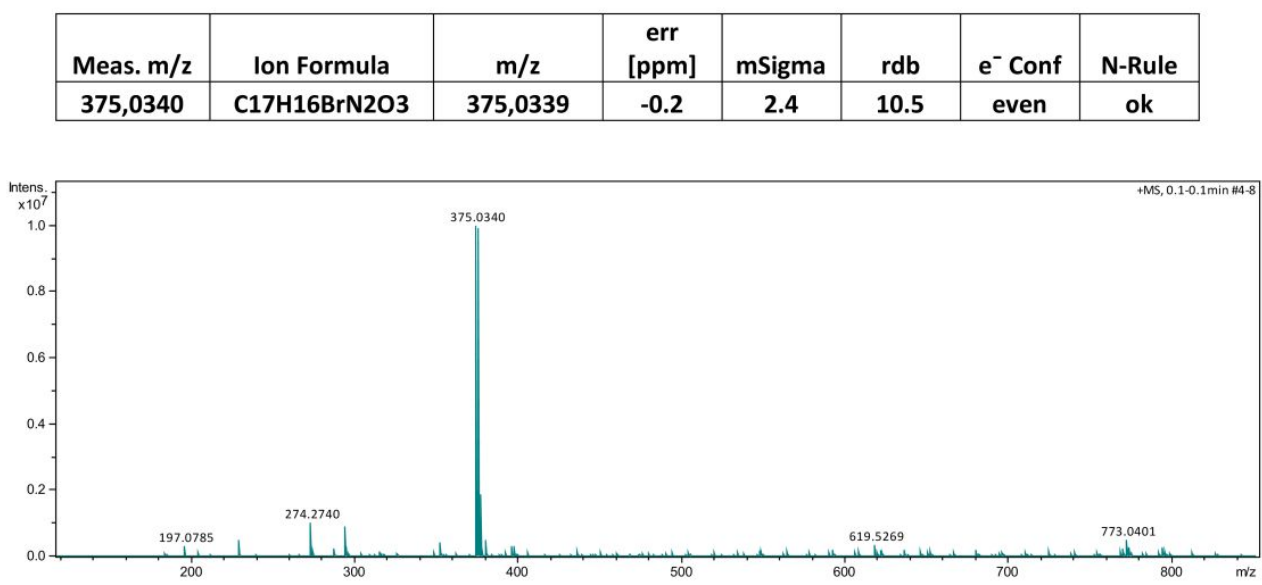

Figure S15. UHPLC-QTOF-MS of 8.

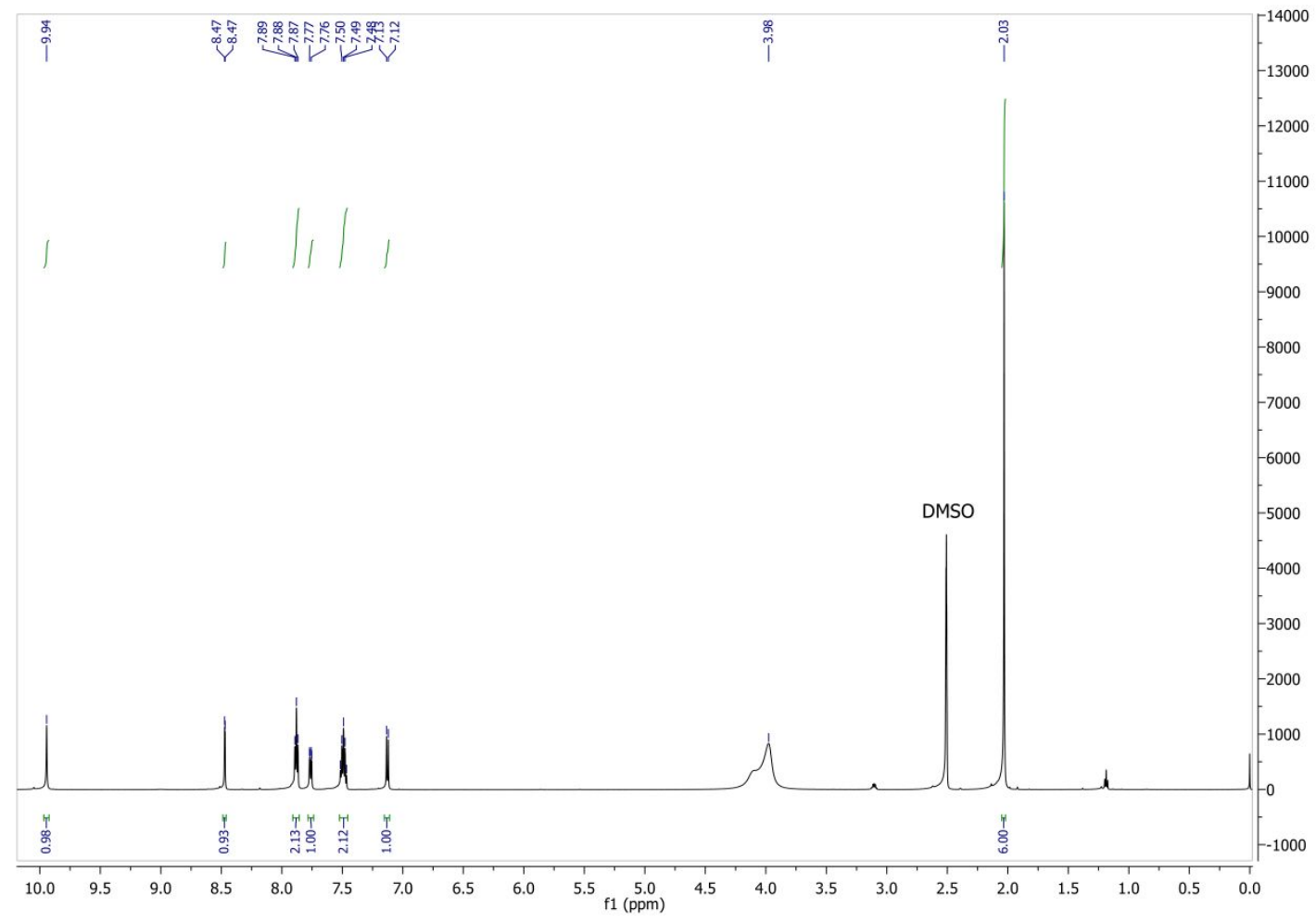

Figure S16. ${ }^{1} \mathrm{H}-\mathrm{NMR}$ spectrum of 9. 


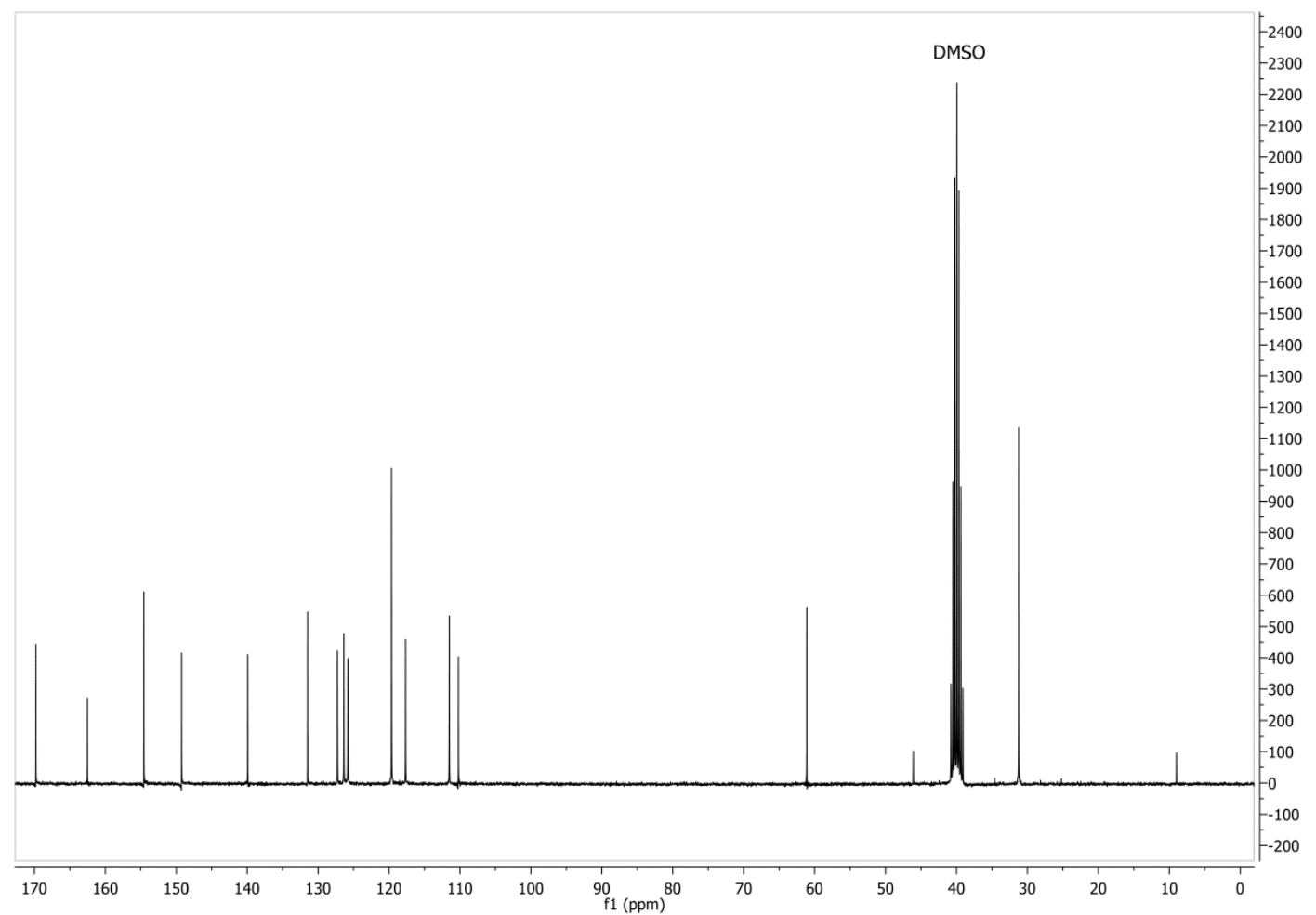

Figure S17. ${ }^{13} \mathrm{C}-\mathrm{NMR}$ spectrum of 9 .

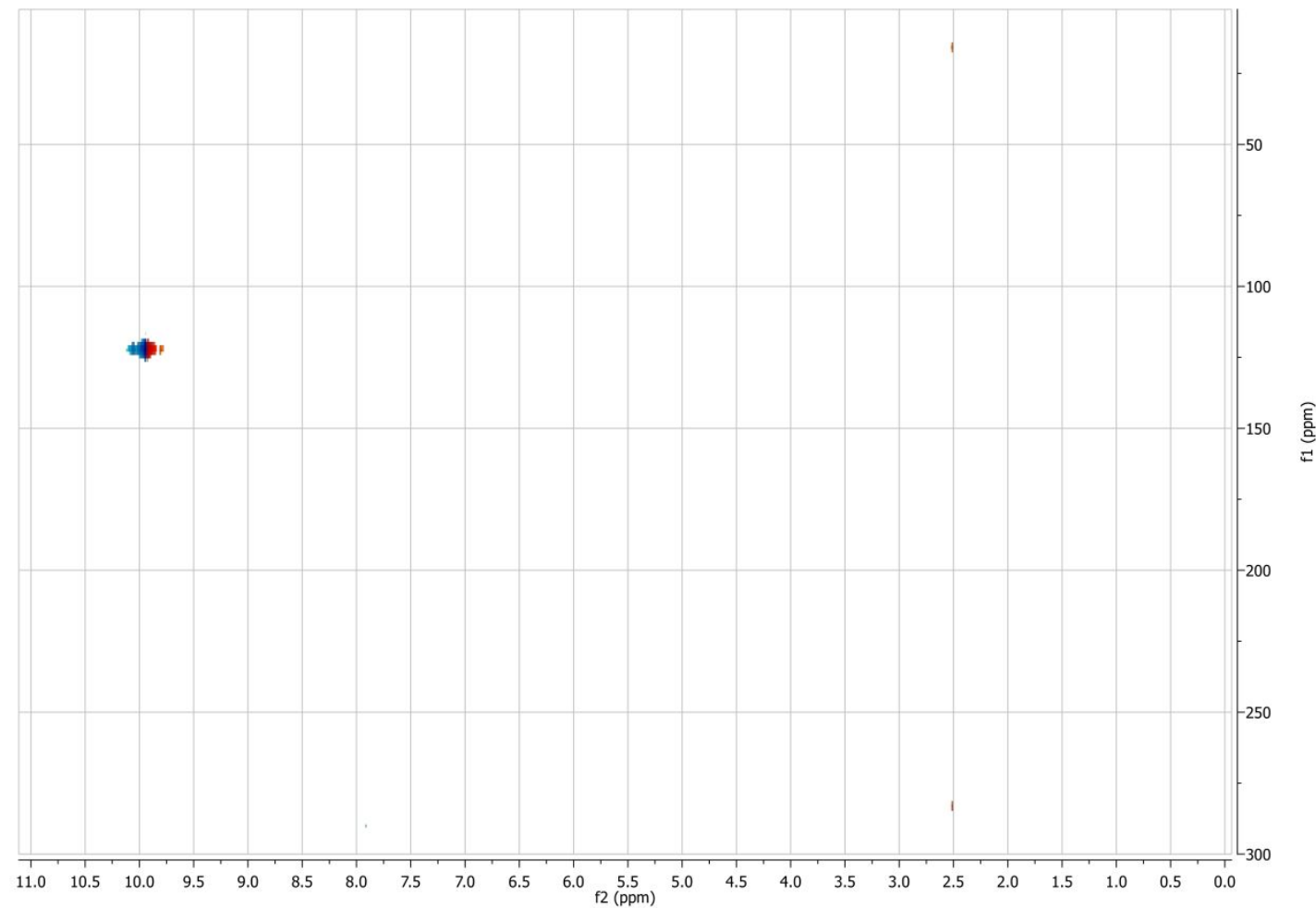

Figure S18. ${ }^{1} \mathrm{H}-{ }^{14} \mathrm{~N}-\mathrm{HMQC}$ spectrum of 9. 


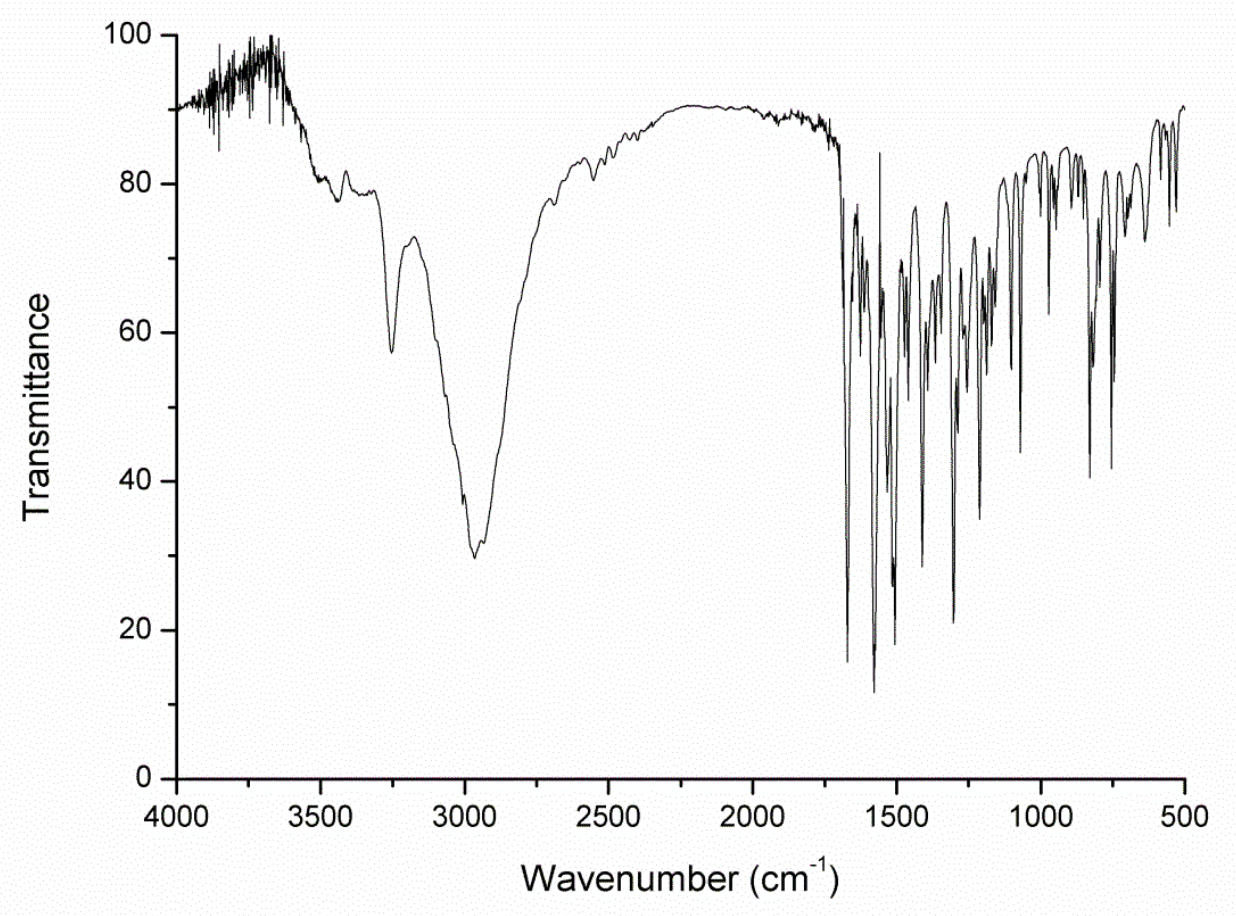

Figure S19. FTIR spectrum 9.

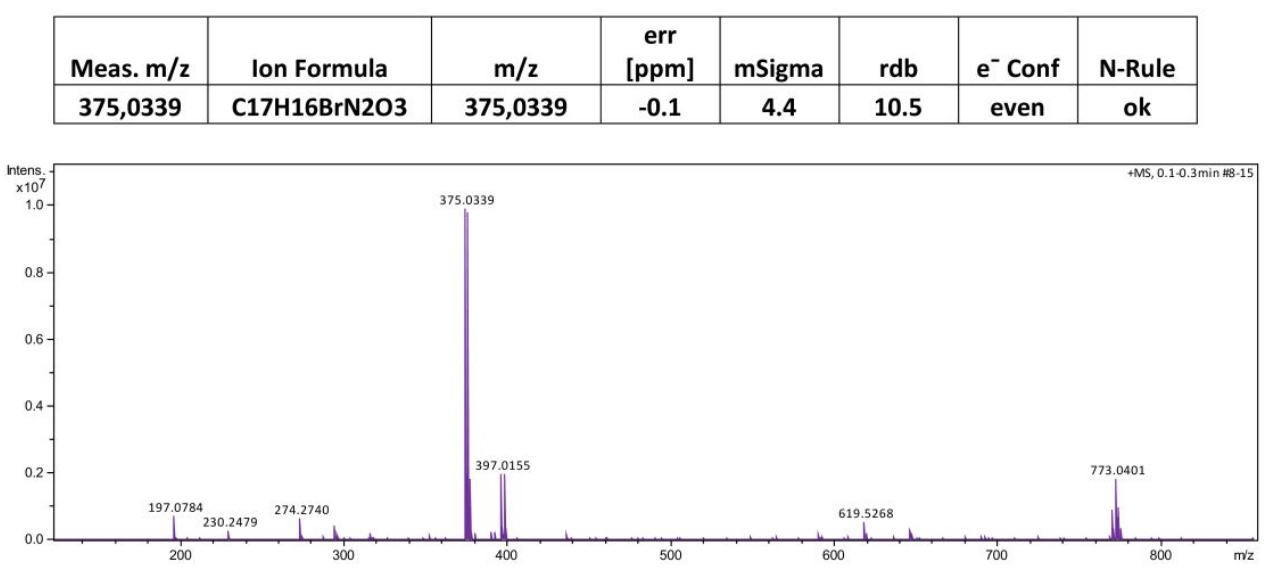

Figure S20. UHPLC-QTOF-MS of 9. 


\section{Polymerization data}

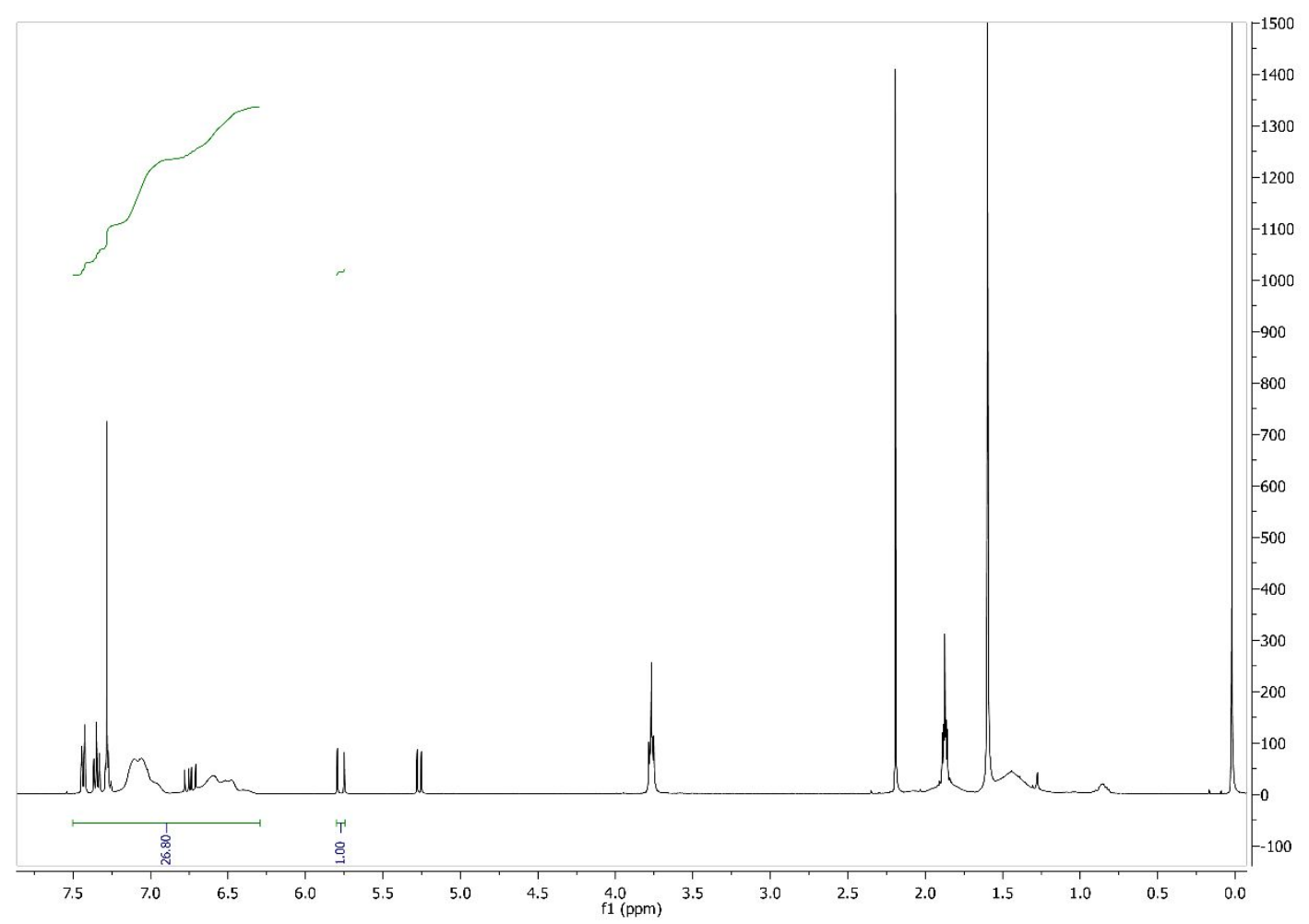

Figure S21. ${ }^{1} \mathrm{H}-\mathrm{NMR}$ of PS6.

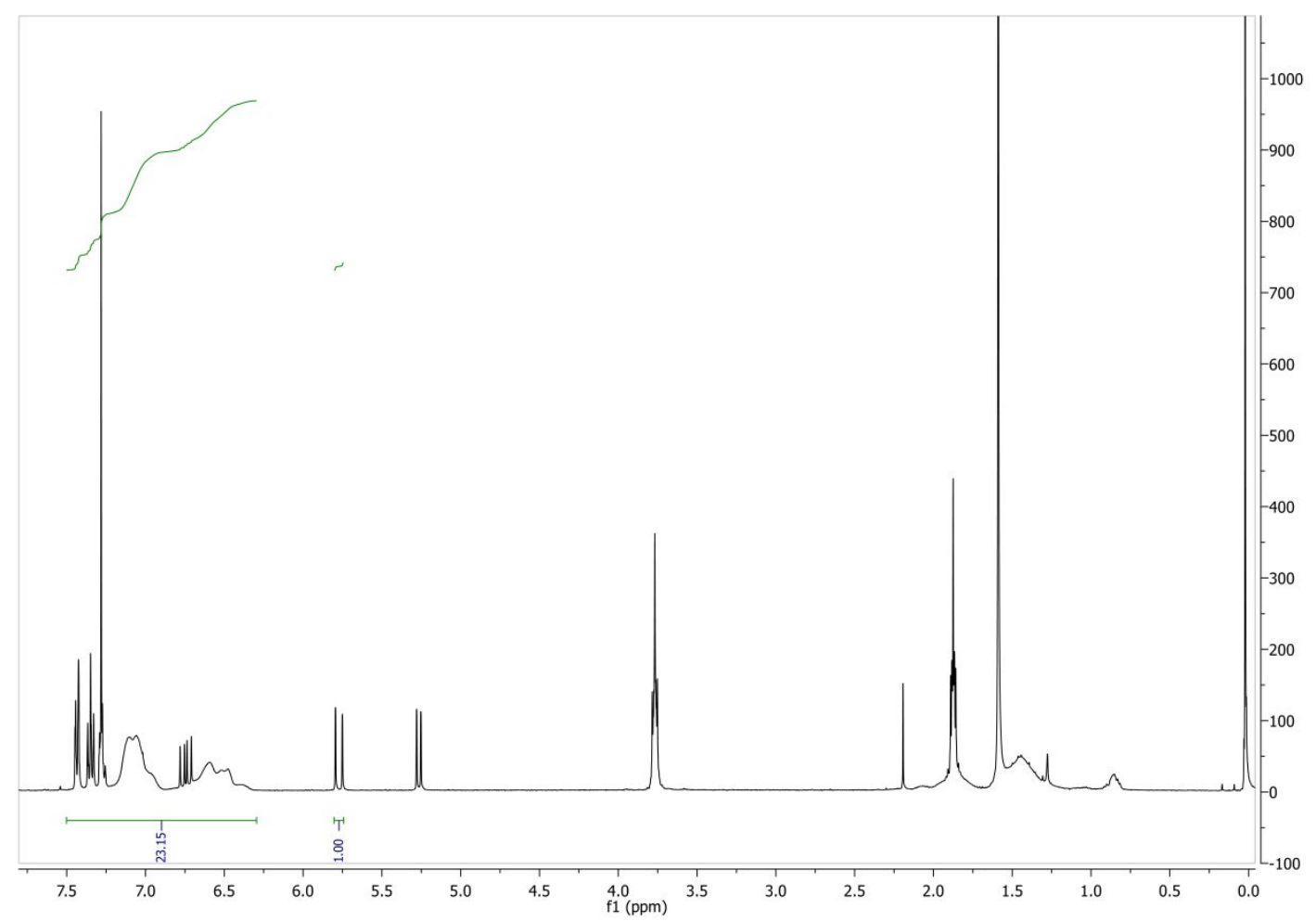

Figure S22. ${ }^{1} \mathrm{H}-\mathrm{NMR}$ spectrum of PS7. 


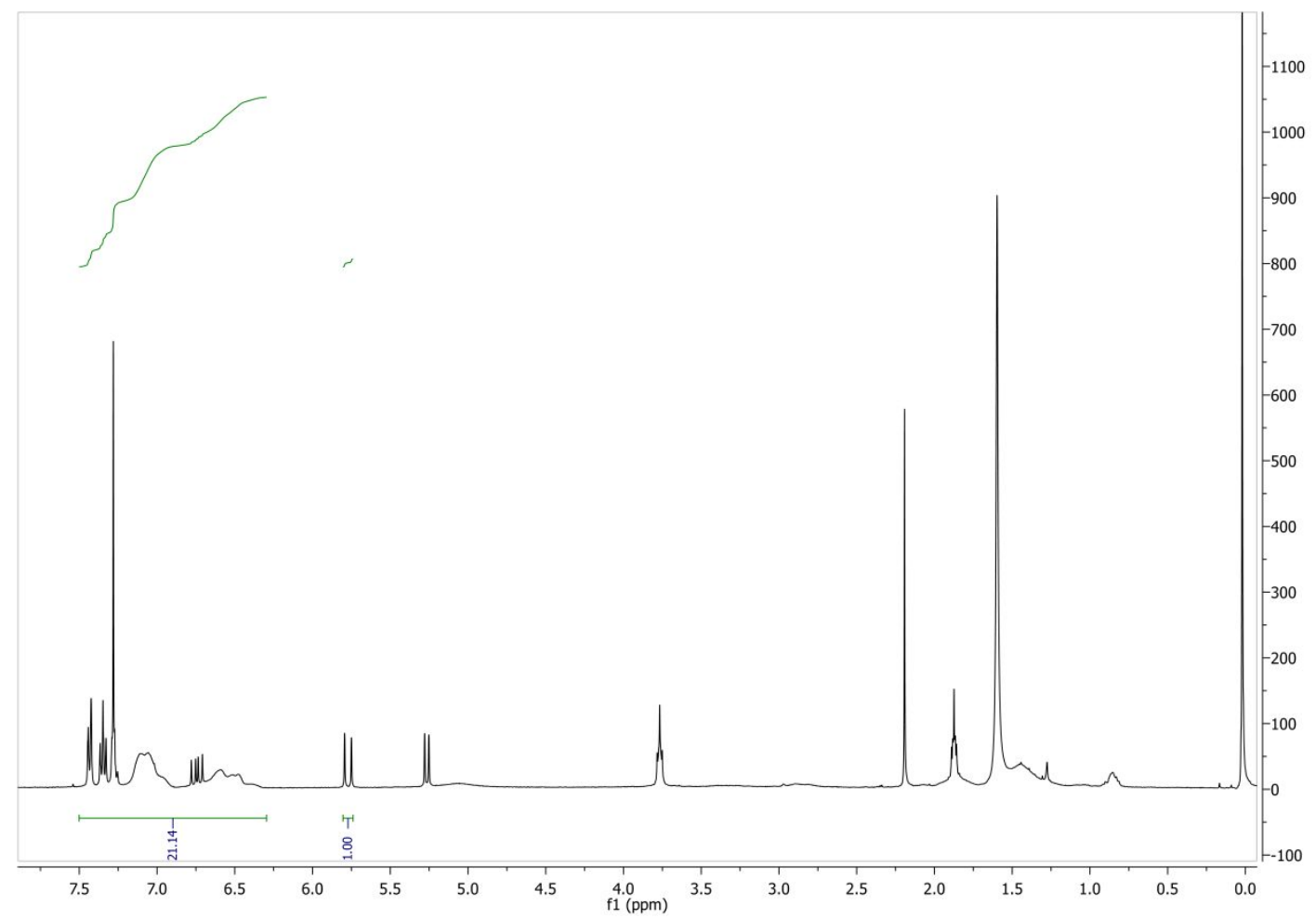

Figure S23. ${ }^{~} \mathrm{H}-\mathrm{NMR}$ spectrum of PS8.

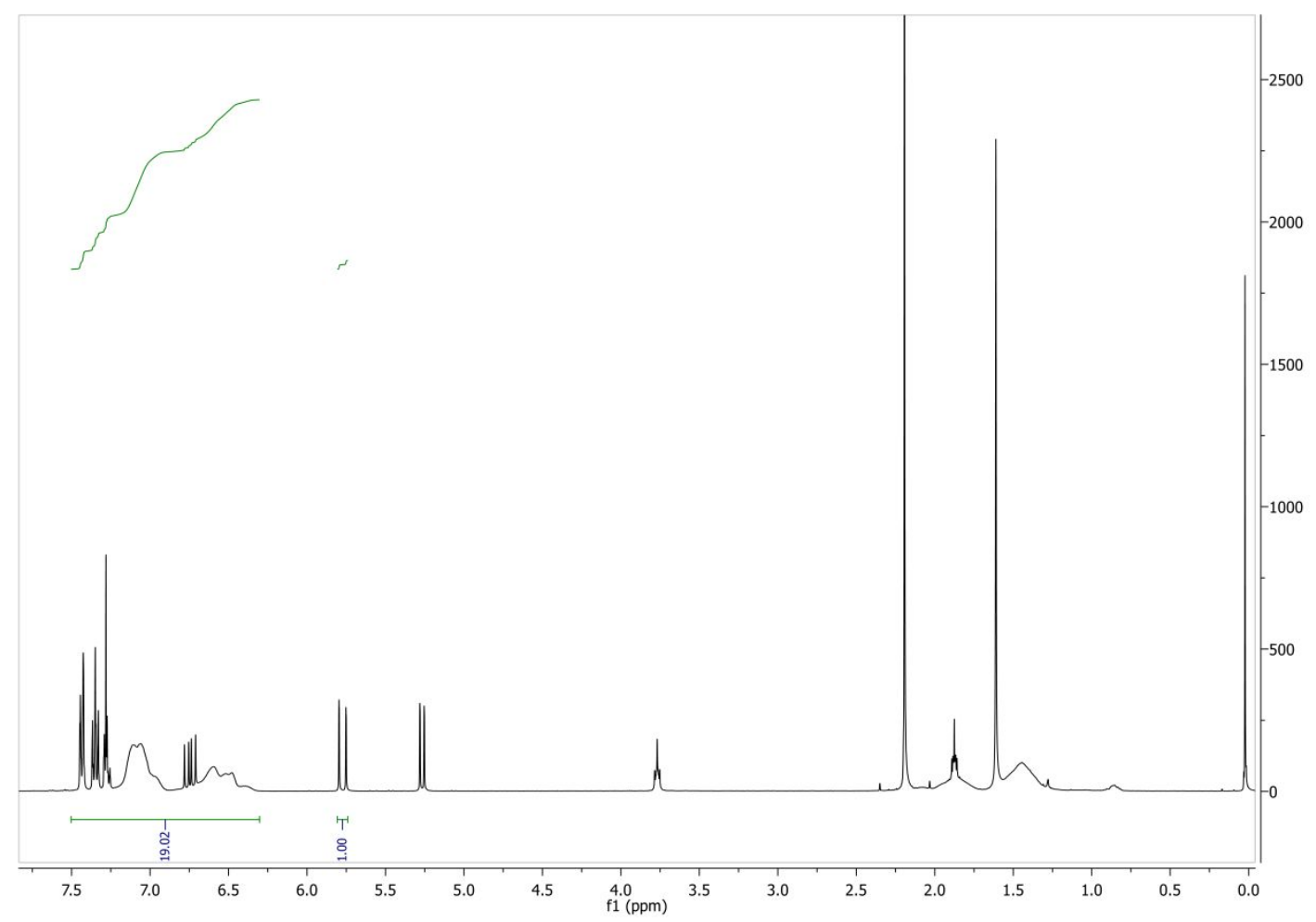

Figure S24. ${ }^{1} \mathrm{H}-\mathrm{NMR}$ spectrum of PS9. 


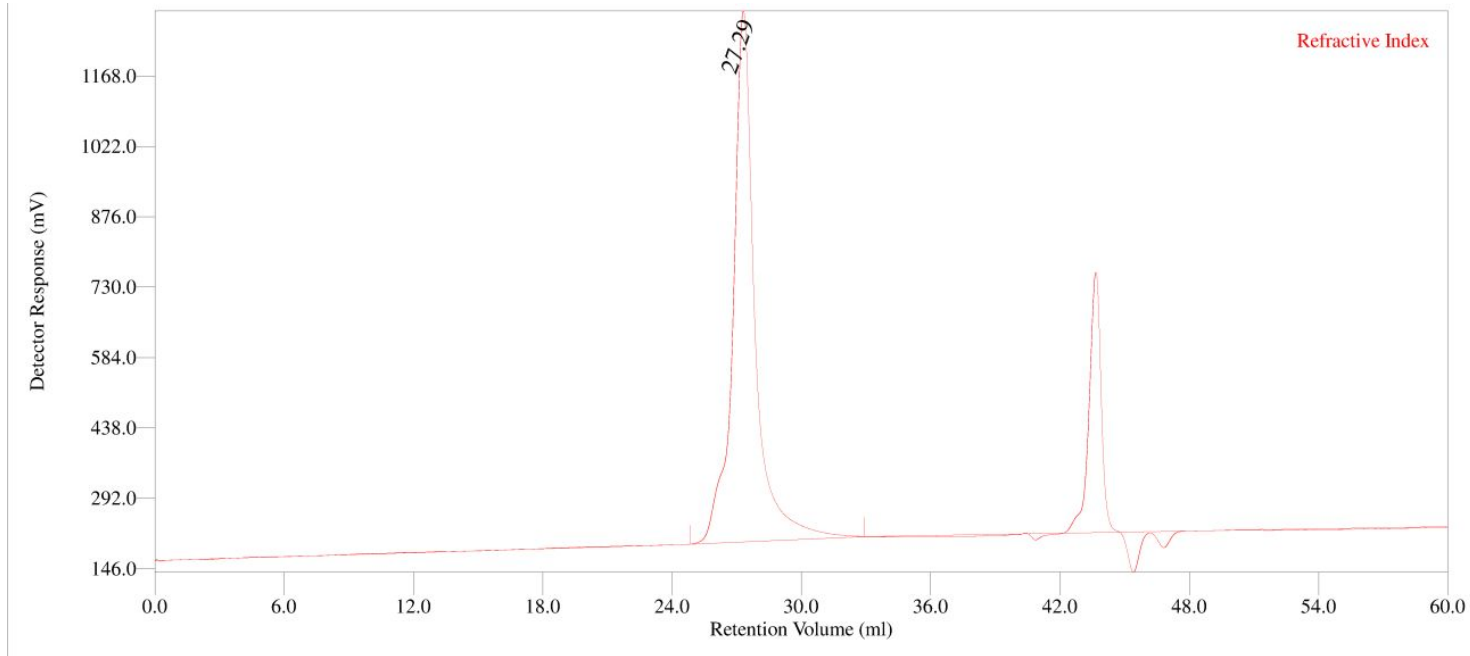

Conventional Calibration - Homopolymers : Results

\begin{tabular}{|l|r|}
\hline Peak RV - (ml) & 27.293 \\
\hline
\end{tabular}

\begin{tabular}{l|r}
\hline Mn - (Daltons) & 86,519 \\
\hline
\end{tabular}

\begin{tabular}{l|l}
$\mathrm{Mw}$ - (Daltons) $\quad 104,732$ \\
\hline
\end{tabular}

\begin{tabular}{l|l}
$\mathrm{Mz}$ - (Daltons) & 119,905 \\
\hline
\end{tabular}

\begin{tabular}{l|r}
\hline Mz - (Daltons) & 119,905 \\
\hline Mp - (Daltons) & 104,324 \\
\hline
\end{tabular}

\begin{tabular}{lr}
\hline Mp - (Daltons) & 104,324 \\
\hline $\mathrm{Mw} / \mathrm{Mn}$ & 1.211 \\
\hline
\end{tabular}

Percent Above Mw: $0 \quad 0.000$

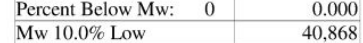

\begin{tabular}{|l|r|}
\hline Mw $10.0 \%$ Low & 40,868 \\
\hline Mw $10.0 \%$ High & 189,561 \\
\hline
\end{tabular}

\begin{tabular}{|r|r|}
\hline RI Area - $(\mathrm{mvml})$ & 1297.89 \\
\hline
\end{tabular}

UV Area - $(\mathrm{mvml})$

0.00

\begin{tabular}{|l|l|}
\hline Annotation & \\
\hline Method File & Conventional_PS_Julho_2016-0017.vcm \\
\hline
\end{tabular}

\begin{tabular}{l|r} 
Method File & Conventional_PS_Julho_2016-0017.vcm \\
\hline Limits File & RP6 01-Conventional PS Julho 2016-0017-0004.lim
\end{tabular}

\begin{tabular}{l|l}
\hline Date Acquired & Aug 15, 2016 - 12:42:48 \\
\hline Solch &
\end{tabular}

Solvent

\begin{tabular}{l|l}
\hline Acquisition Operator & admin : Administrator \\
\hline Caluation Operator & admin : Administrator
\end{tabular}

KF806L 805L 804L 803L

\begin{tabular}{l|l} 
System & GPC Advanced
\end{tabular}

Flow Rate - $(\mathrm{ml} / \mathrm{min}) \quad 1.000$

\begin{tabular}{lr} 
Inj Volume - (ul) & 100.0 \\
\hline
\end{tabular}

\begin{tabular}{lr} 
Volume Increment - $(\mathrm{ml})$ & 0.00333 \\
\hline
\end{tabular}

(otector Temp. - (deg C)

45.0
45.0

OmniSEC Build Number

346
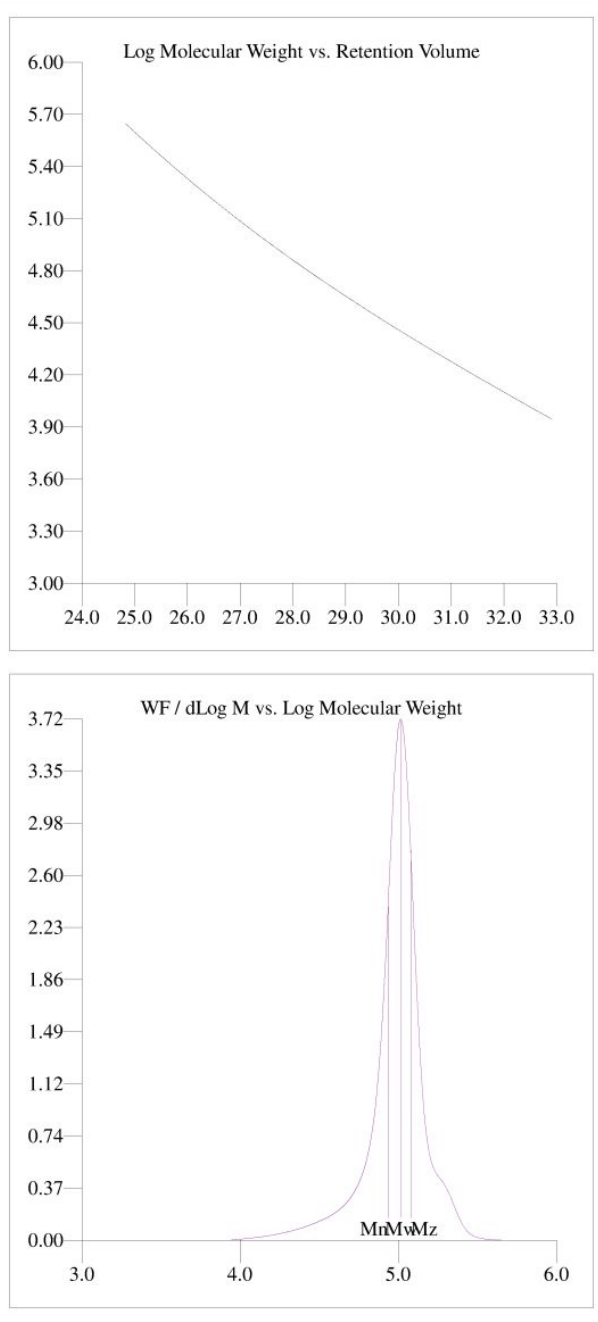

Figure S25. SEC results of PS6. 


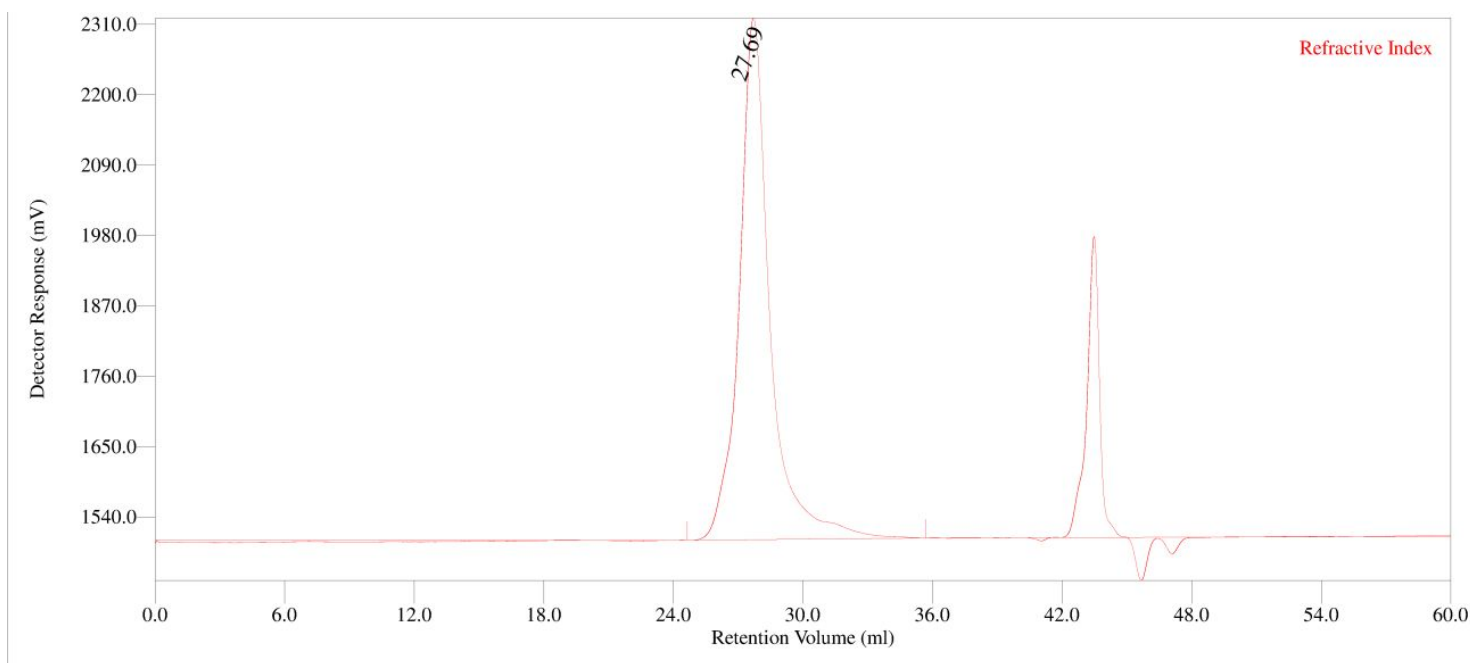

Conventional Calibration - Homopolymers : Results

\begin{tabular}{|l|r|}
\hline Peak RV - (ml) & 27.690 \\
\hline
\end{tabular}

\begin{tabular}{lr}
\hline Mn - (Daltons) & 62,474 \\
\hline Mw - (Daln)
\end{tabular}

Mw - (Daltons) $\quad 85,007$

\begin{tabular}{lr} 
Mz - (Daltons) & $\mathbf{8 5}, 461$ \\
\hline $\mathrm{Mp}$-(Dalons) & 84,870
\end{tabular}

\begin{tabular}{|l|r}
\hline Mp - (Daltons) & 84,870 \\
\hline $\mathrm{Mw} / \mathrm{Mn}$ & 1.361 \\
\hline
\end{tabular}

\begin{tabular}{lll}
\hline Mw/Mn & & 1.361 \\
\hline Percent Above Mw: & 0 & 0.000 \\
\hline
\end{tabular}

\begin{tabular}{lrr} 
Percent Below Mw: & 0 & 0.000 \\
\hline Mw $10.0 \%$ Low & 25,767 \\
\hline
\end{tabular}

\begin{tabular}{lr} 
Mw $10.0 \%$ Low & 0.000 \\
\hline & 25,767 \\
\hline
\end{tabular}

Mw 10.0\% High $\quad 165,779$

\begin{tabular}{|r|r|}
\hline RI Area - $(\mathrm{mvml})$ & 1339.96 \\
\hline
\end{tabular}

\begin{tabular}{lr}
\hline UV Area - $(\mathrm{mvml})$ & 0.00
\end{tabular}

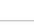

\begin{tabular}{|l|r|}
\hline Annotation & \\
\hline Method File & Conventional_PS_Julho_2016-0017.vcm \\
\hline Limits File & RP8 01-Conventional_PS_Julho_2016-0017-0004.lim \\
\hline Date Acquired & Aug 16, 2016 - 20:47:38 \\
\hline Solvent & THF \\
\hline Acquisition Operator & admin : Administrator \\
\hline Calculation Operator & admin : Administrator \\
\hline Column Set & KF806L,805L,804L, 803L \\
\hline System & GPC Advanced \\
\hline Flow Rate - (ml/min) & 1.000 \\
\hline Inj Volume - (ul) & 100.0 \\
\hline Volume Increment - (ml) & 0.00333 \\
\hline Detector Temp. - (deg C) & 45.0 \\
\hline Column Temp. - (deg C) & 45.0 \\
\hline OmniSEC Build Number & 346 \\
\hline
\end{tabular}
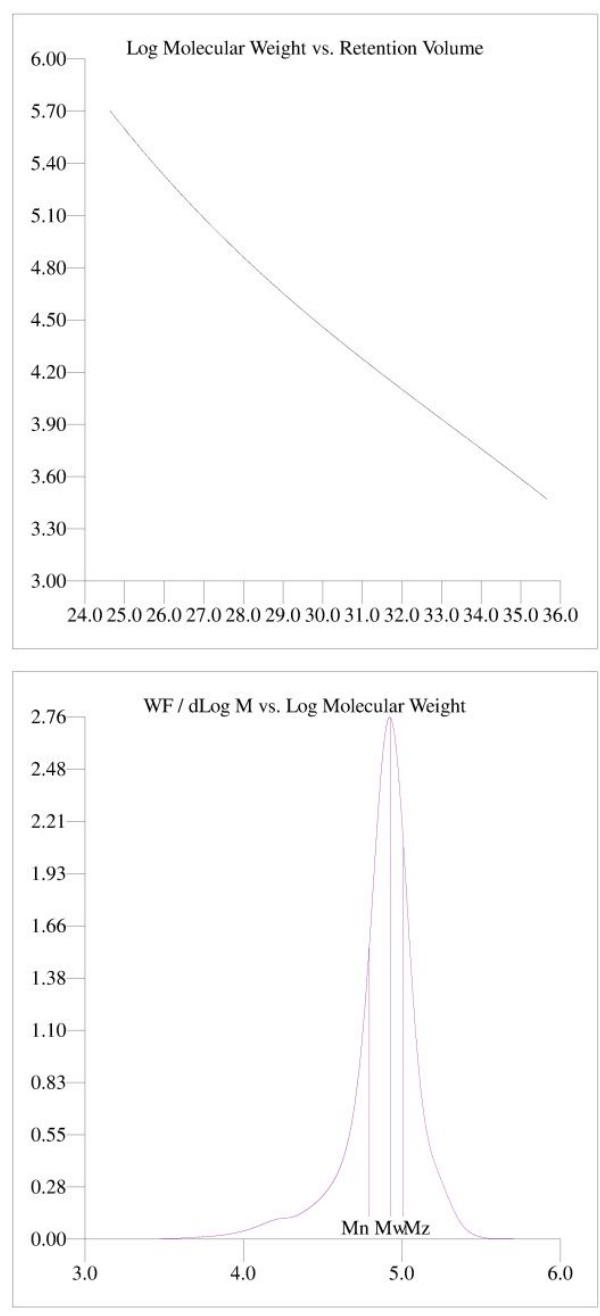

Figure S26. SEC results of PS7. 


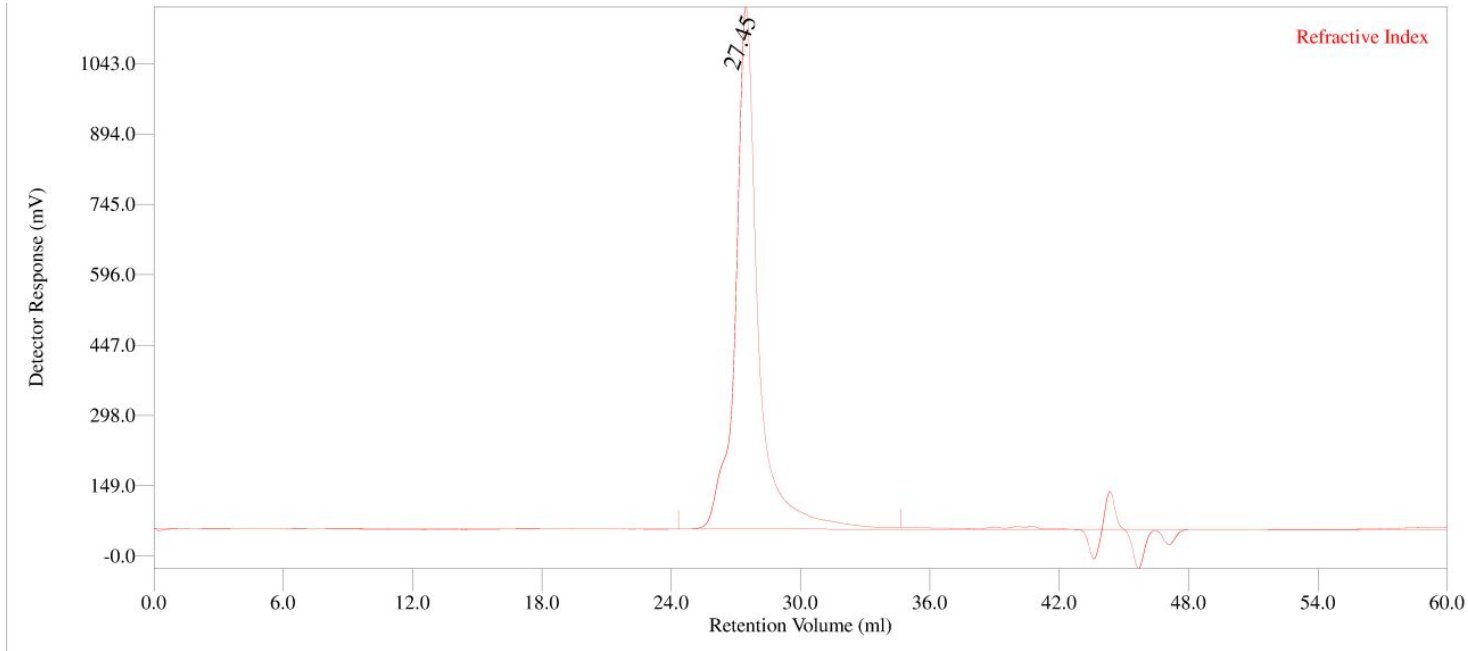

Conventional Calibration - Homopolymers : Results

\begin{tabular}{|l|r|}
\hline Peak RV - $(\mathrm{ml})$ & 27.453 \\
\hline
\end{tabular}

\begin{tabular}{lr}
\hline Mn - (Daltons) & 71,395 \\
\hline
\end{tabular}

\begin{tabular}{l} 
Mw - (Daltons) \\
\hline Mw
\end{tabular}

\begin{tabular}{lr}
\hline Mz - (Daltons) & 109,840 \\
\hline
\end{tabular}

\begin{tabular}{lr} 
Mp - (Daltons) & 95,845 \\
\hline
\end{tabular}

\begin{tabular}{lr}
\hline $\mathrm{Mp}$ - (Daltons) & 95,845 \\
\hline $\mathrm{Mw} / \mathrm{Mn}$ & 1.326 \\
\hline
\end{tabular}

\begin{tabular}{llr}
\hline Mw/Mn & & 1.326 \\
\hline Percent Above Mw: & 0 & 0.000 \\
\hline
\end{tabular}

\begin{tabular}{lll}
\hline Percent Above Mw: & 0 & 0.000 \\
\hline Percent Below Mw: & 0 & 30,887 \\
\hline
\end{tabular}

\begin{tabular}{|l|l|}
\hline Mw 10.0\% Low & 30,887 \\
\hline
\end{tabular}

Mw 10.0\% High

\begin{tabular}{|l|r|}
\hline RI Area - $(\mathrm{mvml})$ & 1340.28 \\
\hline UV Area - $(\mathrm{mvml})$ & 0.00
\end{tabular}

UV Area - $(\mathrm{mvml})$

Annotation

\begin{tabular}{l|l} 
& \\
\hline Method File & Conventional_PS_Julho_2016-0017.vcm
\end{tabular}

Limits File RP6_01-Conventional_PS_Julho_2016-0017-0000.lim

Date Acquired Aug 30, 2016 - 15:43:11

\begin{tabular}{l|r} 
Solvent & THF \\
\hline Acquisition Operator & admin : Administrator
\end{tabular}

Calculation Operator admin : Administrator

\begin{tabular}{l|r}
\hline Column Set & KF806L,805L,804L,803L \\
\hline System & GPC Advanced \\
\hline
\end{tabular}

\begin{tabular}{l|r}
\hline System & GPC Advanced \\
\hline Flow Rate - $(\mathrm{ml} / \mathrm{min})$ & 1.000 \\
\hline
\end{tabular}

\begin{tabular}{l|r}
\hline Inj Volume - (ul) & 100.0 \\
\hline Volume Increment - $(\mathrm{ml})$ & 0.00333 \\
\hline
\end{tabular}

\begin{tabular}{lr} 
Detector Temp. - $(\operatorname{deg} C)$ & 45.0 \\
\hline Column Temp. - $(\operatorname{deg} C)$ & 45.0 \\
\hline OmniSEC Build Number
\end{tabular}

346
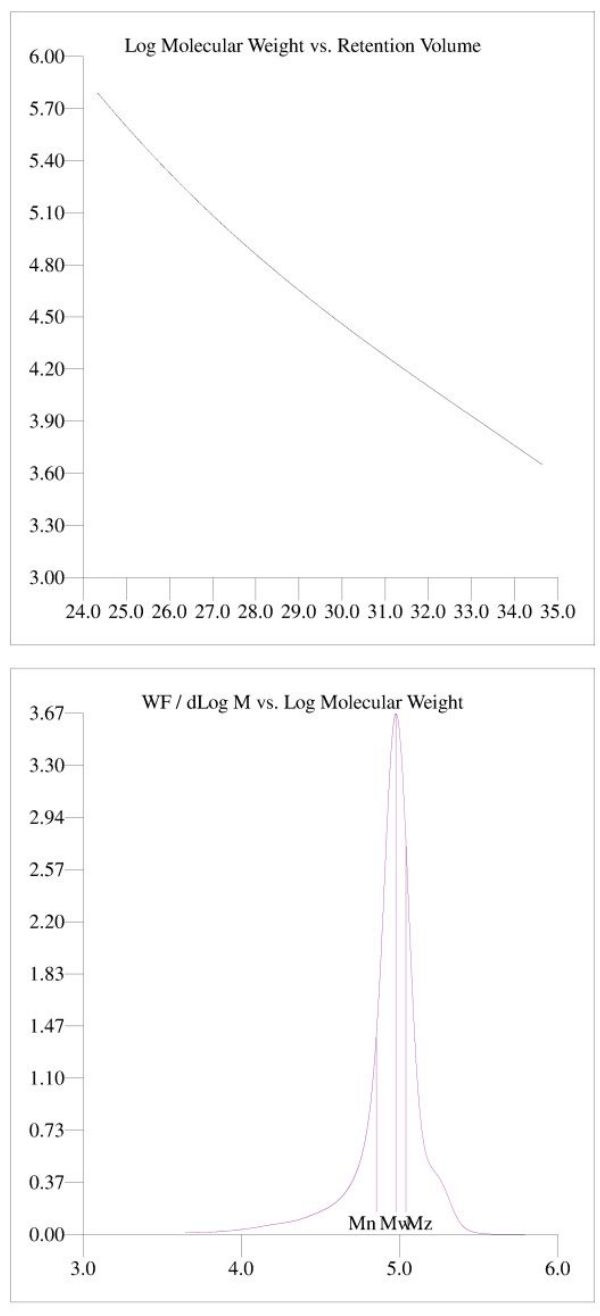

Figure S27. SEC results of PS8. 


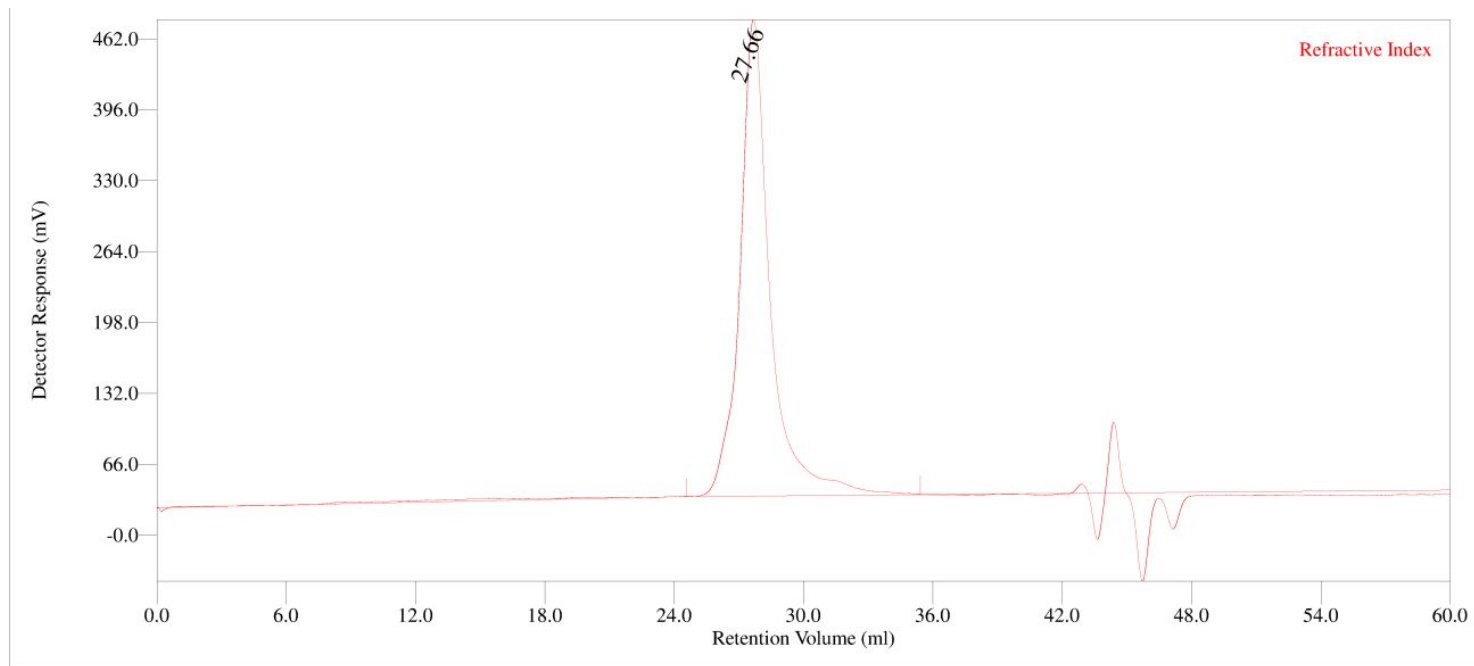

Conventional Calibration - Homopolymers : Results

\begin{tabular}{|l|r|}
\hline Peak RV - $(\mathrm{ml})$ & 27.660 \\
\hline
\end{tabular}

\begin{tabular}{l|r}
\hline Mn - (Daltons) & 60,699 \\
\hline Mw - (Dalos)
\end{tabular}

\begin{tabular}{l}
$\mathrm{Mw}$ - (Daltons) $\quad 84,541$ \\
\hline $\mathrm{Mz}$ - (Datons)
\end{tabular}

\begin{tabular}{lr}
$\mathrm{Mz}$ - (Daltons) & 101,738 \\
\hline $\mathrm{Mp}-($ Dalons & 86,393
\end{tabular}

\begin{tabular}{lr}
\hline Mp - (Daltons) & 86,220 \\
\hline$M w / M n$
\end{tabular}

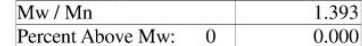

\begin{tabular}{ll|l}
\hline Percent Below Mw: & 0 & 0.000 \\
\hline
\end{tabular}

\begin{tabular}{|r|r}
\hline Mw $10.0 \%$ Low & 24,414 \\
\hline
\end{tabular}

Mw 10.0\% High

\begin{tabular}{lr}
\hline RI Area - $(\mathrm{mvml})$ & 720.26 \\
\hline
\end{tabular}

UV Area - $(\mathrm{mvml})$

\begin{tabular}{|l|l|}
\hline Annotation & \\
\hline Method File & \\
\hline
\end{tabular}

Limits File RP8_01-Conventional_PS_Julho_2016-0017-0000.lim

Date Acquired Aug 31, 2016 - 11:58:23

\begin{tabular}{lr} 
Date Acquired & Aug 31, 2016-11.58.23 \\
\hline Solvent & THF
\end{tabular}

Acquisition Operator admin : Administrator

Calculation Operator admin :

KF806L,805L,804L,803L

GPC Advanced
1.000

\begin{tabular}{|l|l}
\hline Flow Rate - $(\mathrm{ml} / \mathrm{min})$ & 1.000 \\
\hline Inj Volume - (ul) & 100.0 \\
\hline Vlute $-(\mathrm{m})$ & 0.0333
\end{tabular}

\begin{tabular}{|rr}
\hline Inj Volume - (ul) & 100.0 \\
\hline Volume Increment - $(\mathrm{ml})$ & 0.00333 \\
\hline Detector Temp. $-(\operatorname{deg} \mathrm{C})$ & 45.0
\end{tabular}

\begin{tabular}{|r|r|}
\hline Detector Temp. - $(\operatorname{deg} C)$ & 45.0 \\
\hline Column Temp. $-(\operatorname{deg} C)$ & 45.0 \\
\hline
\end{tabular}

45.0
346

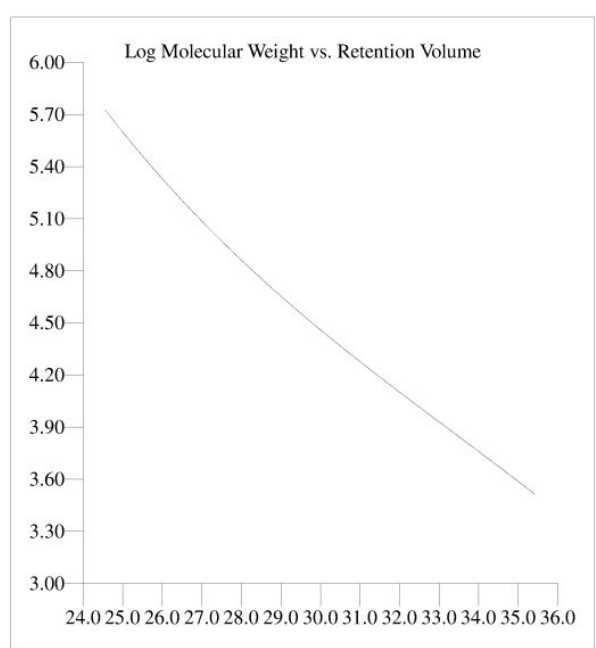

OmniSEC Build Number

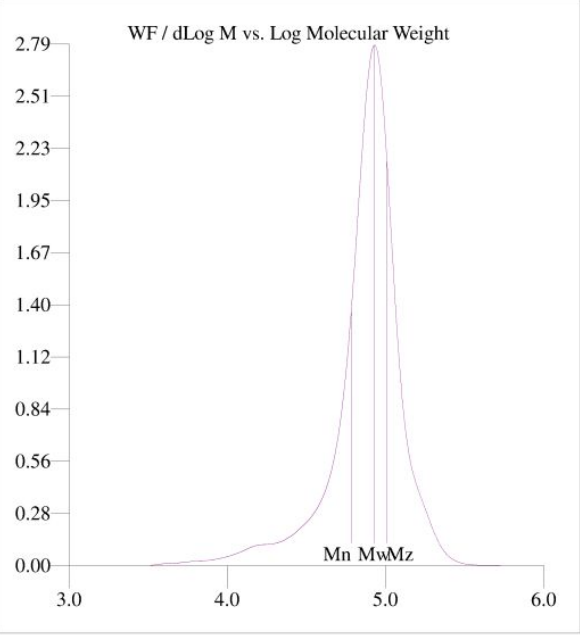

Figure S28. SEC results of PS9. 


\section{Data Resume of DRX analysis}

\section{Initiator 6}

Table S1. Crystal data.

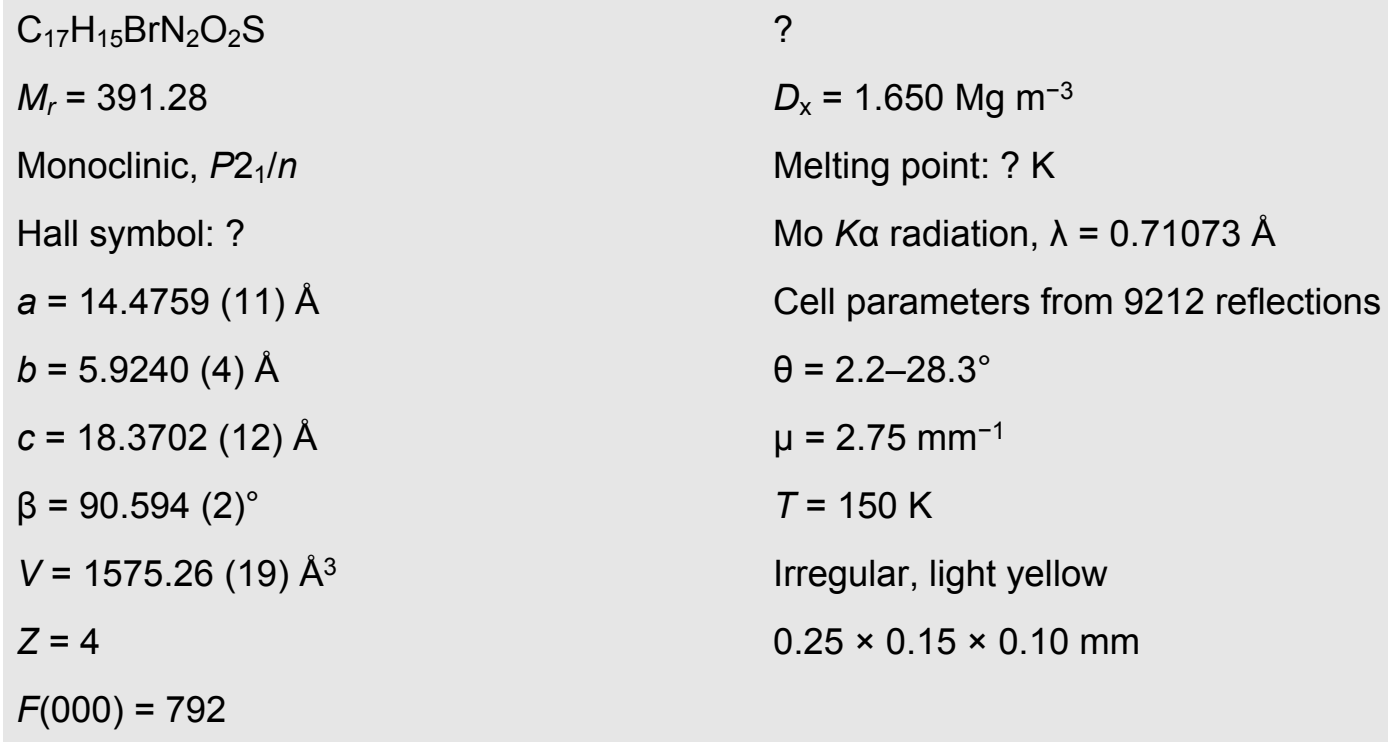

Table S2. Data collection.

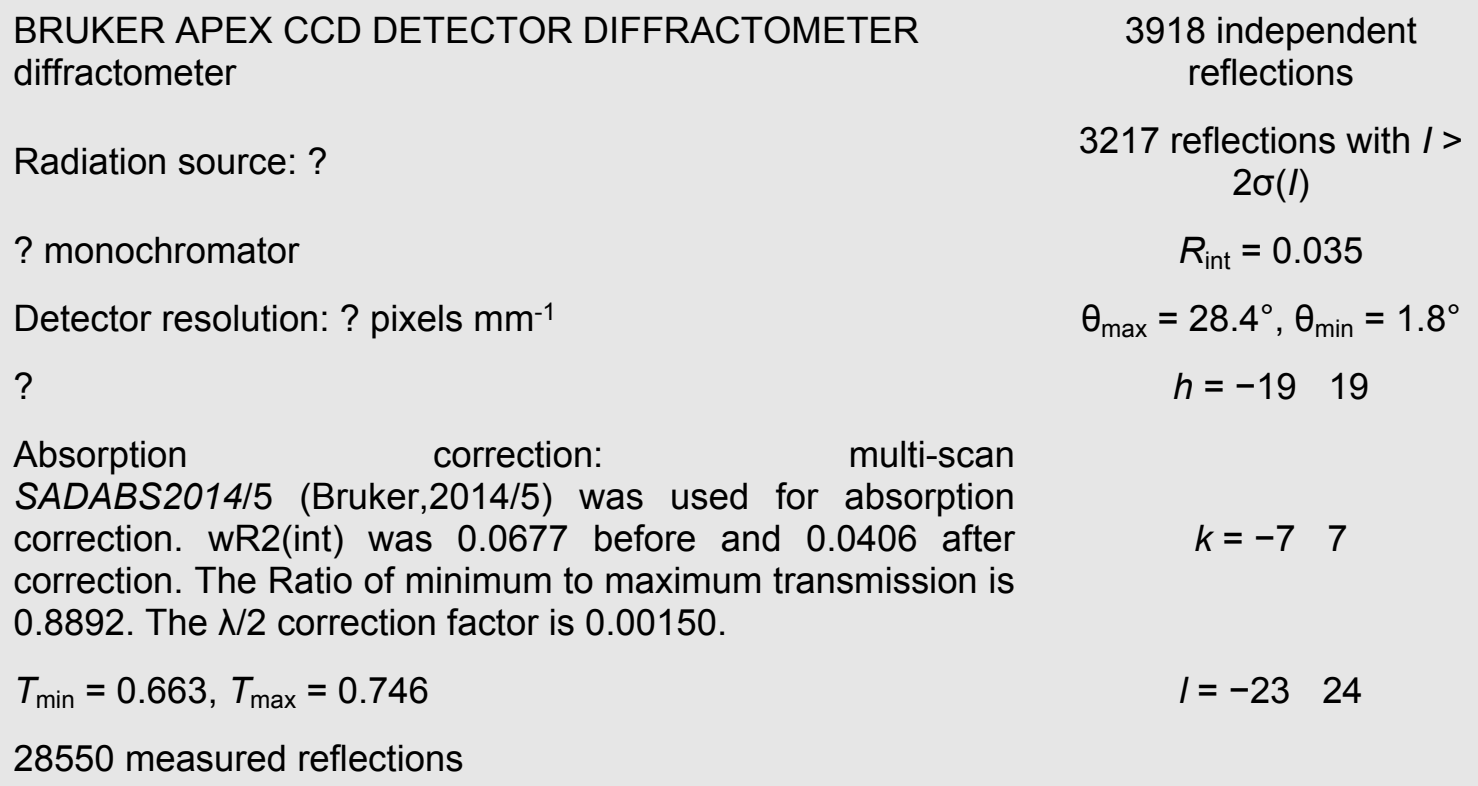
correction. wR2(int) was 0.0677 before and 0.0406 after correction. The Ratio of minimum to maximum transmission is

3918 independent reflections

3217 reflections with $I>$ $2 \sigma(I)$

$R_{\text {int }}=0.035$

$\theta_{\max }=28.4^{\circ}, \theta_{\min }=1.8^{\circ}$

$h=-19 \quad 19$

$$
k=-7 \quad 7
$$

$T_{\min }=0.663, T_{\max }=0.746$

$I=-23 \quad 24$

28550 measured reflections

Table S3. Refinement. 
Refinement on $F^{2}$

Least-squares matrix: full

$R\left[F^{2}>2 \sigma\left(F^{2}\right)\right]=0.047$

$w R\left(F^{2}\right)=0.149$

$S=1.03$

3918 reflections

211 parameters

0 restraints

? constraints
Secondary atom site location: ?

Hydrogen site location: inferred from neighbouring sites

$\mathrm{H}$-atom parameters constrained

$w=1 /\left[\sigma^{2}\left(F_{0}^{2}\right)+(0.0807 P)^{2}+4.0349 P\right]$

where $P=\left(F_{\mathrm{o}}^{2}+2 F_{\mathrm{c}}^{2}\right) / 3$

$(\Delta / \sigma)_{\max }<0.001$

$\Delta \rho_{\max }=0.73$ e $\AA^{-3}$

$\Delta \rho_{\min }=-1.43$ e $\AA^{-3}$

Extinction correction: none

Extinction coefficient: ?

Primary atom site location: dual

Table S4. Fractional atomic coordinates and isotropic or equivalent isotropic displacement parameters $\left(\AA^{2}\right)$.

$\begin{array}{ccccc} & x & y & z & U_{\text {iso }}^{*} / U_{\text {eq }} \\ \text { Br1 } & 0.91135(3) & -0.20301(7) & 0.49282(3) & 0.04566(18) \\ \text { C1 } & 0.3640(2) & 1.2180(6) & 0.2143(2) & 0.0236(7) \\ \text { H1A } & 0.355363 & 1.323848 & 0.175988 & 0.028^{*} \\ \text { C2 } & 0.3151(2) & 1.2444(6) & 0.27970(19) & 0.0227(7) \\ \text { H2A } & 0.274067 & 1.368229 & 0.284948 & 0.027^{*} \\ \text { C3 } & 0.3260(2) & 1.0936(5) & 0.33604(18) & 0.0209(6) \\ \text { H3 } & 0.292977 & 1.111560 & 0.380078 & 0.025^{*} \\ \text { C4 } & 0.3870(2) & 0.9132(5) & 0.32680(16) & 0.0167(6) \\ \text { C5 } & 0.43651(19) & 0.8866(5) & 0.26213(16) & 0.0155(5) \\ \text { C6 } & 0.4239(2) & 1.0404(6) & 0.20552(18) & 0.0210(6) \\ \text { H6 } & 0.456471 & 1.022722 & 0.161252 & 0.025^{*} \\ \text { C7 } & 0.49230(19) & 0.5927(5) & 0.32422(16) & 0.0146(5) \\ \text { C8 } & 0.54791(18) & 0.3954(5) & 0.34036(15) & 0.0140(5) \\ \text { C9 } & 0.5406(2) & 0.2793(5) & 0.40651(16) & 0.0158(6) \\ \text { H9 } & 0.496514 & 0.328840 & 0.440992 & 0.019^{*} \\ \text { C10 } & 0.5953(2) & 0.0955(5) & 0.42314(16) & 0.0163(6) \\ \text { H10 } & 0.587991 & 0.018027 & 0.467998 & 0.020^{*} \\ \text { C11 } & 0.66139(19) & 0.0243(5) & 0.37341(16) & 0.0142(5) \\ \text { C12 } & 0.66850(19) & 0.1331(5) & 0.30617(16) & 0.0160(6) \\ \text { H12 } & 0.711786 & 0.080842 & 0.271505 & 0.019^{*} \\ & & & & \end{array}$




$\begin{array}{ccccc}\text { C13 } & 0.61298(19) & 0.3162(5) & 0.28986(16) & 0.0145(5) \\ \text { C14 } & 0.7286(2) & -0.2846(5) & 0.44876(17) & 0.0180(6) \\ \text { C15 } & 0.8165(2) & -0.4259(5) & 0.45956(17) & 0.0188(6) \\ \text { C16 } & 0.8519(2) & -0.5395(6) & 0.39095(19) & 0.0262(7) \\ \text { H16A } & 0.869463 & -0.423951 & 0.355495 & 0.039^{*} \\ \text { H16B } & 0.803255 & -0.635199 & 0.370012 & 0.039^{*} \\ \text { H16C } & 0.905915 & -0.632414 & 0.403173 & 0.039^{*} \\ \text { C17 } & 0.8051(2) & -0.5938(7) & 0.52052(19) & 0.0284(7) \\ \text { H17A } & 0.864126 & -0.669557 & 0.530100 & 0.043^{*} \\ \text { H17B } & 0.758442 & -0.706060 & 0.506528 & 0.043^{*} \\ \text { H17C } & 0.785203 & -0.514805 & 0.564544 & 0.043^{*} \\ \text { N1 } & 0.49585(17) & 0.7023(4) & 0.26229(14) & 0.0164(5) \\ \text { N2 } & 0.72595(17) & -0.1489(5) & 0.38840(14) & 0.0167(5) \\ \text { H2 } & 0.768822 & -0.171862 & 0.355611 & 0.020^{*} \\ \text { O1 } & 0.62354(15) & 0.4180(4) & 0.22394(11) & 0.0200(5) \\ \text { H1 } & 0.587511 & 0.528758 & 0.220705 & 0.030^{*} \\ \text { O2 } & 0.66657(17) & -0.2955(4) & 0.49275(14) & 0.0286(6) \\ \text { S1 } & 0.41504(5) & 0.70149(13) & 0.38770(4) & 0.01790(18) \\ & & & & \end{array}$

Table S5. Atomic displacement parameters $\left(\AA^{2}\right)$.

$\begin{array}{lcccccc} & U^{11} & U^{22} & U^{33} & U^{12} & U^{13} & U^{23} \\ \text { Br1 } & 0.0339(2) & 0.0282(2) & 0.0744(4) & -0.00283(15) & -0.0247(2) & -0.00583(19) \\ \text { C1 } & 0.0219(15) & 0.0192(15) & 0.0294(17) & -0.0012(12) & -0.0059(13) & 0.0039(13) \\ \text { C2 } & 0.0204(15) & 0.0176(14) & 0.0299(17) & 0.0038(11) & -0.0061(12) & -0.0064(12) \\ \text { C3 } & 0.0183(13) & 0.0201(15) & 0.0241(16) & 0.0027(12) & -0.0007(11) & -0.0094(12) \\ \text { C4 } & 0.0159(13) & 0.0161(13) & 0.0178(14) & -0.0015(11) & -0.0027(10) & -0.0028(11) \\ \text { C5 } & 0.0122(12) & 0.0153(13) & 0.0189(14) & -0.0022(10) & 0.0005(10) & -0.0036(11) \\ \text { C6 } & 0.0182(14) & 0.0232(15) & 0.0217(16) & 0.0001(12) & 0.0025(11) & 0.0025(12) \\ \text { C7 } & 0.0120(12) & 0.0160(13) & 0.0159(14) & -0.0022(10) & 0.0024(10) & -0.0014(11) \\ \text { C8 } & 0.0118(12) & 0.0155(13) & 0.0148(14) & -0.0020(10) & 0.0021(10) & -0.0014(11) \\ \text { C9 } & 0.0158(13) & 0.0187(14) & 0.0131(13) & 0.0006(11) & 0.0045(10) & -0.0021(11) \\ \text { C10 } & 0.0173(13) & 0.0194(14) & 0.0121(13) & 0.0000(11) & 0.0025(10) & -0.0013(11) \\ \text { C11 } & 0.0129(12) & 0.0152(13) & 0.0144(13) & -0.0002(10) & -0.0008(10) & -0.0009(10) \\ \text { C12 } & 0.0139(12) & 0.0209(14) & 0.0132(14) & 0.0005(11) & 0.0031(10) & 0.0005(11) \\ \text { C13 } & 0.0126(12) & 0.0185(14) & 0.0126(13) & -0.0020(10) & 0.0029(10) & 0.0004(11) \\ \text { C14 } & 0.0178(14) & 0.0186(14) & 0.0177(14) & 0.0010(11) & 0.0001(11) & -0.0003(11)\end{array}$




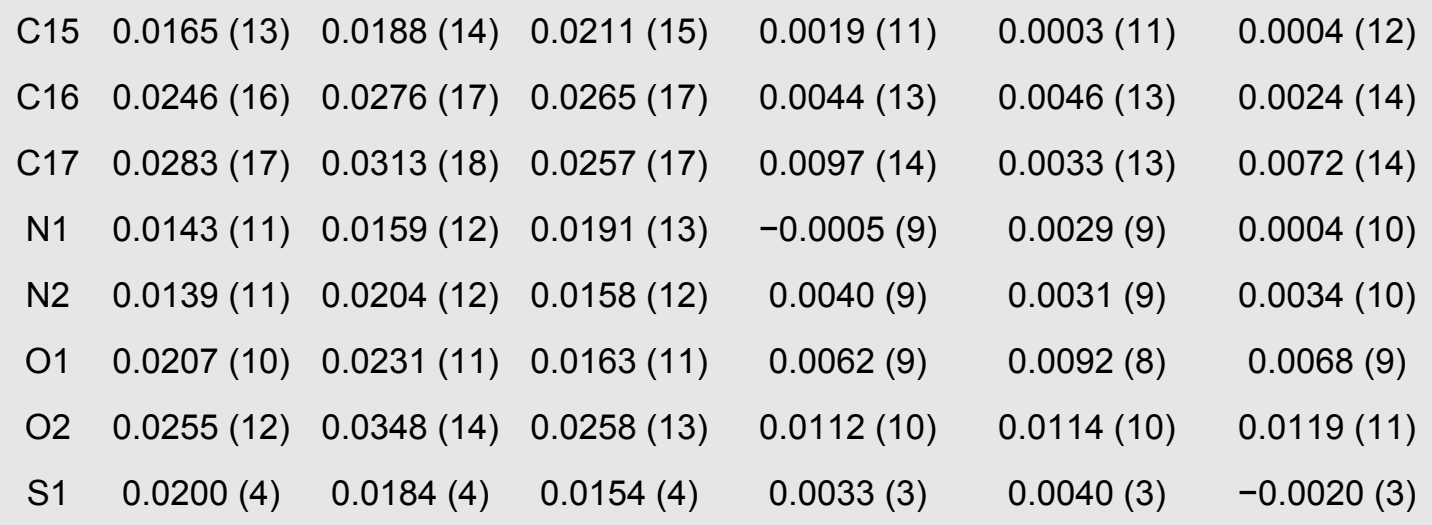

Table S6. Geometric parameters $\left(\AA,{ }^{\circ}\right)$.

\begin{tabular}{|c|c|c|c|}
\hline $\mathrm{Br} 1-\mathrm{C} 15$ & $1.996(3)$ & $\mathrm{C} 10-\mathrm{H} 10$ & 0.9500 \\
\hline $\mathrm{C} 1-\mathrm{H} 1 \mathrm{~A}$ & 0.9500 & C10-C11 & $1.395(4)$ \\
\hline $\mathrm{C} 1-\mathrm{C} 2$ & $1.408(5)$ & C11-C12 & $1.398(4)$ \\
\hline $\mathrm{C} 1-\mathrm{C} 6$ & $1.375(5)$ & $\mathrm{C} 11-\mathrm{N} 2$ & $1.413(4)$ \\
\hline $\mathrm{C} 2-\mathrm{H} 2 \mathrm{~A}$ & 0.9500 & $\mathrm{C} 12-\mathrm{H} 12$ & 0.9500 \\
\hline $\mathrm{C} 2-\mathrm{C} 3$ & $1.375(5)$ & $\mathrm{C} 12-\mathrm{C} 13$ & $1.381(4)$ \\
\hline $\mathrm{C} 3-\mathrm{H} 3$ & 0.9500 & $\mathrm{C} 13-\mathrm{O} 1$ & $1.363(3)$ \\
\hline $\mathrm{C} 3-\mathrm{C} 4$ & $1.397(4)$ & C14-C15 & $1.533(4)$ \\
\hline $\mathrm{C} 4-\mathrm{C} 5$ & $1.403(4)$ & $\mathrm{C} 14-\mathrm{N} 2$ & $1.370(4)$ \\
\hline $\mathrm{C} 4-\mathrm{S} 1$ & $1.726(3)$ & $\mathrm{C} 14-\mathrm{O} 2$ & $1.216(4)$ \\
\hline $\mathrm{C} 5-\mathrm{C} 6$ & $1.393(4)$ & C15-C16 & $1.523(4)$ \\
\hline $\mathrm{C} 5-\mathrm{N} 1$ & $1.389(4)$ & $\mathrm{C} 15-\mathrm{C} 17$ & $1.508(5)$ \\
\hline $\mathrm{C} 6-\mathrm{H} 6$ & 0.9500 & C16-H16A & 0.9800 \\
\hline $\mathrm{C} 7-\mathrm{C} 8$ & $1.448(4)$ & $\mathrm{C} 16-\mathrm{H} 16 \mathrm{~B}$ & 0.9800 \\
\hline $\mathrm{C} 7-\mathrm{N} 1$ & $1.311(4)$ & $\mathrm{C} 16-\mathrm{H} 16 \mathrm{C}$ & 0.9800 \\
\hline $\mathrm{C} 7-\mathrm{S} 1$ & $1.747(3)$ & $\mathrm{C} 17-\mathrm{H} 17 \mathrm{~A}$ & 0.9800 \\
\hline $\mathrm{C} 8-\mathrm{C} 9$ & $1.401(4)$ & $\mathrm{C} 17-\mathrm{H} 17 \mathrm{~B}$ & 0.9800 \\
\hline $\mathrm{C} 8-\mathrm{C} 13$ & $1.409(4)$ & $\mathrm{C} 17-\mathrm{H} 17 \mathrm{C}$ & 0.9800 \\
\hline $\mathrm{C} 9-\mathrm{H} 9$ & 0.9500 & $\mathrm{~N} 2-\mathrm{H} 2$ & 0.8800 \\
\hline $\mathrm{C} 9-\mathrm{C} 10$ & $1.379(4)$ & $\mathrm{O} 1-\mathrm{H} 1$ & 0.8400 \\
\hline $\mathrm{C} 2-\mathrm{C} 1-\mathrm{H} 1 \mathrm{~A}$ & 119.7 & $\mathrm{C} 11-\mathrm{C} 12-\mathrm{H} 12$ & 119.8 \\
\hline $\mathrm{C} 6-\mathrm{C} 1-\mathrm{H} 1 \mathrm{~A}$ & 119.7 & $\mathrm{C} 13-\mathrm{C} 12-\mathrm{C} 11$ & $120.4(3)$ \\
\hline $\mathrm{C} 6-\mathrm{C} 1-\mathrm{C} 2$ & $120.5(3)$ & $\mathrm{C} 13-\mathrm{C} 12-\mathrm{H} 12$ & 119.8 \\
\hline $\mathrm{C} 1-\mathrm{C} 2-\mathrm{H} 2 \mathrm{~A}$ & 119.5 & $\mathrm{C} 12-\mathrm{C} 13-\mathrm{C} 8$ & $120.7(3)$ \\
\hline $\mathrm{C} 3-\mathrm{C} 2-\mathrm{C} 1$ & $121.0(3)$ & $\mathrm{O} 1-\mathrm{C} 13-\mathrm{C} 8$ & $121.3(3)$ \\
\hline
\end{tabular}




\begin{tabular}{|c|c|c|c|}
\hline $\mathrm{C} 3-\mathrm{C} 2-\mathrm{H} 2 \mathrm{~A}$ & 119.5 & $\mathrm{O} 1-\mathrm{C} 13-\mathrm{C} 12$ & $118.0(3)$ \\
\hline $\mathrm{C} 2-\mathrm{C} 3-\mathrm{H} 3$ & 120.9 & $\mathrm{~N} 2-\mathrm{C} 14-\mathrm{C} 15$ & $116.2(3)$ \\
\hline $\mathrm{C} 2-\mathrm{C} 3-\mathrm{C} 4$ & $118.2(3)$ & $\mathrm{O} 2-\mathrm{C} 14-\mathrm{C} 15$ & $120.2(3)$ \\
\hline $\mathrm{C} 4-\mathrm{C} 3-\mathrm{H} 3$ & 120.9 & $\mathrm{O} 2-\mathrm{C} 14-\mathrm{N} 2$ & $123.6(3)$ \\
\hline $\mathrm{C} 3-\mathrm{C} 4-\mathrm{C} 5$ & $121.2(3)$ & $\mathrm{C} 14-\mathrm{C} 15-\mathrm{Br} 1$ & $104.2(2)$ \\
\hline $\mathrm{C} 3-\mathrm{C} 4-\mathrm{S} 1$ & $128.4(2)$ & $\mathrm{C} 16-\mathrm{C} 15-\mathrm{Br} 1$ & $108.0(2)$ \\
\hline $\mathrm{C} 5-\mathrm{C} 4-\mathrm{S} 1$ & $110.4(2)$ & $\mathrm{C} 16-\mathrm{C} 15-\mathrm{C} 14$ & $114.8(3)$ \\
\hline $\mathrm{C} 6-\mathrm{C} 5-\mathrm{C} 4$ & $119.6(3)$ & $\mathrm{C} 17-\mathrm{C} 15-\mathrm{Br} 1$ & $106.8(2)$ \\
\hline $\mathrm{N} 1-\mathrm{C} 5-\mathrm{C} 4$ & $114.1(3)$ & $\mathrm{C} 17-\mathrm{C} 15-\mathrm{C} 14$ & $111.0(3)$ \\
\hline $\mathrm{N} 1-\mathrm{C} 5-\mathrm{C} 6$ & $126.3(3)$ & $\mathrm{C} 17-\mathrm{C} 15-\mathrm{C} 16$ & $111.3(3)$ \\
\hline $\mathrm{C} 1-\mathrm{C} 6-\mathrm{C} 5$ & $119.4(3)$ & $\mathrm{C} 15-\mathrm{C} 16-\mathrm{H} 16 \mathrm{~A}$ & 109.5 \\
\hline $\mathrm{C} 1-\mathrm{C} 6-\mathrm{H} 6$ & 120.3 & $\mathrm{C} 15-\mathrm{C} 16-\mathrm{H} 16 \mathrm{~B}$ & 109.5 \\
\hline $\mathrm{C} 5-\mathrm{C} 6-\mathrm{H} 6$ & 120.3 & $\mathrm{C} 15-\mathrm{C} 16-\mathrm{H} 16 \mathrm{C}$ & 109.5 \\
\hline $\mathrm{C} 8-\mathrm{C} 7-\mathrm{S} 1$ & $121.3(2)$ & $\mathrm{H} 16 \mathrm{~A}-\mathrm{C} 16-\mathrm{H} 16 \mathrm{~B}$ & 109.5 \\
\hline $\mathrm{N} 1-\mathrm{C} 7-\mathrm{C} 8$ & $123.4(3)$ & $\mathrm{H} 16 \mathrm{~A}-\mathrm{C} 16-\mathrm{H} 16 \mathrm{C}$ & 109.5 \\
\hline $\mathrm{N} 1-\mathrm{C} 7-\mathrm{S} 1$ & $115.3(2)$ & $\mathrm{H} 16 \mathrm{~B}-\mathrm{C} 16-\mathrm{H} 16 \mathrm{C}$ & 109.5 \\
\hline $\mathrm{C} 9-\mathrm{C} 8-\mathrm{C} 7$ & $121.8(3)$ & C15-C17-H17A & 109.5 \\
\hline $\mathrm{C} 9-\mathrm{C} 8-\mathrm{C} 13$ & $117.7(3)$ & $\mathrm{C} 15-\mathrm{C} 17-\mathrm{H} 17 \mathrm{~B}$ & 109.5 \\
\hline $\mathrm{C} 13-\mathrm{C} 8-\mathrm{C} 7$ & $120.5(3)$ & $\mathrm{C} 15-\mathrm{C} 17-\mathrm{H} 17 \mathrm{C}$ & 109.5 \\
\hline $\mathrm{C} 8-\mathrm{C} 9-\mathrm{H} 9$ & 119.0 & $\mathrm{H} 17 \mathrm{~A}-\mathrm{C} 17-\mathrm{H} 17 \mathrm{~B}$ & 109.5 \\
\hline $\mathrm{C} 10-\mathrm{C} 9-\mathrm{C} 8$ & $122.1(3)$ & $\mathrm{H} 17 \mathrm{~A}-\mathrm{C} 17-\mathrm{H} 17 \mathrm{C}$ & 109.5 \\
\hline $\mathrm{C} 10-\mathrm{C} 9-\mathrm{H} 9$ & 119.0 & $\mathrm{H} 17 \mathrm{~B}-\mathrm{C} 17-\mathrm{H} 17 \mathrm{C}$ & 109.5 \\
\hline $\mathrm{C} 9-\mathrm{C} 10-\mathrm{H} 10$ & 120.3 & $\mathrm{C} 7-\mathrm{N} 1-\mathrm{C} 5$ & $111.2(2)$ \\
\hline $\mathrm{C} 9-\mathrm{C} 10-\mathrm{C} 11$ & $119.3(3)$ & $\mathrm{C} 11-\mathrm{N} 2-\mathrm{H} 2$ & 116.7 \\
\hline $\mathrm{C} 11-\mathrm{C} 10-\mathrm{H} 10$ & 120.3 & $\mathrm{C} 14-\mathrm{N} 2-\mathrm{C} 11$ & $126.7(2)$ \\
\hline $\mathrm{C} 10-\mathrm{C} 11-\mathrm{C} 12$ & $119.8(3)$ & $\mathrm{C} 14-\mathrm{N} 2-\mathrm{H} 2$ & 116.7 \\
\hline $\mathrm{C} 10-\mathrm{C} 11-\mathrm{N} 2$ & $123.3(3)$ & $\mathrm{C} 13-\mathrm{O} 1-\mathrm{H} 1$ & 109.5 \\
\hline $\mathrm{C} 12-\mathrm{C} 11-\mathrm{N} 2$ & $116.9(3)$ & $\mathrm{C} 4-\mathrm{S} 1-\mathrm{C} 7$ & $89.05(14)$ \\
\hline
\end{tabular}

\section{Initiator 7}

Table S7. Crystal data.

$\mathrm{C}_{17} \mathrm{H}_{15} \mathrm{BrN}_{2} \mathrm{O}_{2} \mathrm{~S}$

$M_{r}=391.28$

Monoclinic, $P 2_{1} / C$

Hall symbol: ?
?

$D_{\mathrm{x}}=1.596 \mathrm{Mg} \mathrm{m}^{-3}$

Melting point: ? K

Cu Ka radiation, $\lambda=1.54178 \AA$ 


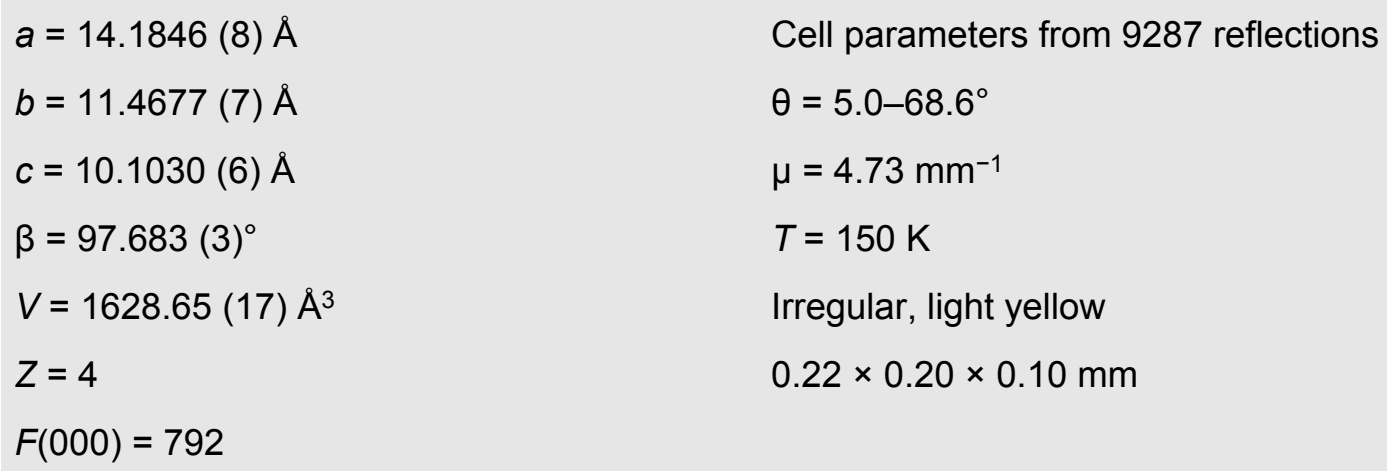

Table S8. Data collection.

\section{BRUKER APEX CCD DETECTOR \\ DIFFRACTOMETER \\ diffractometer}

Radiation source: ?

? monochromator

Detector resolution: ? pixels $\mathrm{mm}^{-1}$

?

Absorption correction: multi-scan

SADABS2014/5 (Bruker,2014/5) was used for absorption correction. wR2(int) was 0.1611 before and 0.0733 after correction. The Ratio of minimum to maximum transmission is 0.6267 . The $\lambda / 2$ correction factor is 0.00150 .

$T_{\min }=0.472, T_{\max }=0.753$

$I=-12 \quad 10$

\section{2 independent reflections}

2691 reflections with $I>2 \sigma(I)$

$R_{\text {int }}=0.053$

$\theta_{\max }=68.6^{\circ}, \theta_{\min }=5.0^{\circ}$

$h=-16 \quad 16$

15704 measured reflections

Table S9. Refinement.

$\begin{array}{ll}\begin{array}{l}\text { Refinement on } F^{2} \\ \text { Least-squares matrix: full }\end{array} & \begin{array}{l}\text { Secondary atom site location: ? } \\ \text { Hydrogen site location: mixed }\end{array} \\ R\left[F^{2}>2 \sigma\left(F^{2}\right)\right]=0.038 & \begin{array}{l}\mathrm{H} \text { atoms treated by a mixture of independent } \\ \text { and constrained refinement }\end{array} \\ w R\left(F^{2}\right)=0.111 & \begin{array}{l}w=1 /\left[\sigma^{2}\left(F_{\mathrm{o}}^{2}\right)+(0.0539 P)^{2}+1.522 P\right] \\ \text { where } P=\left(F_{\mathrm{o}}^{2}+2 F_{\mathrm{c}}^{2}\right) / 3\end{array} \\ S=1.08 & (\Delta / \sigma)_{\max }<0.001 \\ 2952 \text { reflections } & \Delta \rho_{\max }=0.95 \text { e } \AA^{-3} \\ 214 \text { parameters } & \Delta \rho_{\min }=-0.60 \text { e } \AA^{-3} \\ 0 \text { restraints } & \text { Extinction correction: none } \\ ? \text { constraints } & \text { Extinction coefficient: ? }\end{array}$


Primary atom site location: heavy-atom method

Table S10. Fractional atomic coordinates and isotropic or equivalent isotropic displacement parameters $\left(\AA^{2}\right)$.

\begin{tabular}{|c|c|c|c|c|}
\hline & $x$ & $y$ & $z$ & $U_{\text {iso }}^{*} / U_{\text {eq }}$ \\
\hline $\mathrm{Br} 1$ & $0.04022(2)$ & 0.23804 (3) & $0.52353(4)$ & 0.04857 (17) \\
\hline C1 & $0.7581(2)$ & $0.2459(2)$ & $0.3013(3)$ & $0.0309(6)$ \\
\hline $\mathrm{H} 1$ & 0.739585 & 0.175162 & 0.256349 & $0.037^{*}$ \\
\hline $\mathrm{C} 2$ & 0.84070 (19) & $0.3032(3)$ & 0.2797 (3) & $0.0348(6)$ \\
\hline $\mathrm{H} 2$ & 0.878752 & 0.271750 & 0.217813 & $0.042^{*}$ \\
\hline C3 & 0.86895 (17) & $0.4060(3)$ & $0.3470(3)$ & $0.0314(6)$ \\
\hline $\mathrm{H} 3$ & 0.926297 & 0.442930 & 0.330950 & $0.038^{*}$ \\
\hline $\mathrm{C} 4$ & $0.81509(16)$ & $0.4553(2)$ & 0.4367 (3) & $0.0262(5)$ \\
\hline $\mathrm{H} 4$ & 0.834682 & 0.525438 & 0.482356 & $0.031^{*}$ \\
\hline C5 & $0.73088(15)$ & $0.3992(2)$ & $0.4585(2)$ & $0.0211(5)$ \\
\hline C6 & $0.70306(16)$ & $0.2954(2)$ & $0.3913(2)$ & $0.0226(5)$ \\
\hline $\mathrm{C} 7$ & $0.59428(15)$ & $0.36980(19)$ & $0.5399(2)$ & $0.0180(5)$ \\
\hline $\mathrm{C} 8$ & $0.51845(15)$ & $0.39079(19)$ & $0.6218(2)$ & $0.0179(5)$ \\
\hline C9 & $0.52686(16)$ & $0.4834(2)$ & $0.7142(3)$ & $0.0219(5)$ \\
\hline C10 & $0.45609(17)$ & $0.5006(2)$ & $0.7956(2)$ & $0.0238(5)$ \\
\hline $\mathrm{H} 10$ & 0.462445 & 0.561649 & 0.859769 & $0.029^{*}$ \\
\hline C11 & $0.37688(16)$ & $0.4295(2)$ & $0.7838(2)$ & $0.0217(5)$ \\
\hline $\mathrm{H} 11$ & 0.329085 & 0.442224 & 0.839678 & $0.026^{*}$ \\
\hline C12 & $0.36633(14)$ & $0.33926(19)$ & $0.6904(2)$ & $0.0173(5)$ \\
\hline C13 & $0.43736(15)$ & $0.31958(19)$ & $0.6111(2)$ & $0.0169(4)$ \\
\hline $\mathrm{H} 13$ & 0.431024 & 0.257094 & 0.548820 & $0.020^{*}$ \\
\hline C14 & $0.23830(15)$ & $0.2216(2)$ & $0.5703(2)$ & $0.0186(5)$ \\
\hline
\end{tabular}




\begin{tabular}{ccccc} 
C15 & $0.15379(15)$ & $0.1403(2)$ & $0.5843(2)$ & $0.0225(5)$ \\
C16 & $0.1537(2)$ & $0.0395(3)$ & $0.4872(3)$ & $0.0461(8)$ \\
H16A & 0.098558 & -0.010584 & 0.493800 & $0.069^{*}$ \\
H16B & 0.212253 & -0.006022 & 0.508924 & $0.069^{*}$ \\
H16C & 0.150370 & 0.069809 & 0.395976 & $0.069^{*}$ \\
C17 & $0.14324(17)$ & $0.0978(2)$ & $0.7228(3)$ & $0.0300(6)$ \\
H17A & 0.134645 & 0.164646 & 0.780342 & $0.045^{*}$ \\
H17B & 0.200483 & 0.054650 & 0.759370 & $0.045^{*}$ \\
H17C & 0.087719 & 0.046374 & 0.718566 & $0.045^{*}$ \\
N1 & $0.66811(13)$ & $0.43902(17)$ & $0.5427(2)$ & $0.0203(4)$ \\
N2 & $0.28310(14)$ & $0.26899(16)$ & $0.6829(2)$ & $0.0176(4)$ \\
H2A & 0.259296 & 0.255618 & 0.757566 & $0.021^{*}$ \\
O1 & $0.60190(13)$ & $0.55787(16)$ & $0.7292(2)$ & $0.0319(4)$ \\
H1A & $0.634(3)$ & $0.547(3)$ & $0.676(4)$ & $0.044(10)^{*}$ \\
O2 & $0.26181(13)$ & $0.23968(16)$ & $0.45973(18)$ & $0.0268(4)$ \\
S1 & $0.59472(4)$ & $0.24867(5)$ & $0.43493(6)$ & $0.02261(18)$ \\
\hline
\end{tabular}

Table S11. Atomic displacement parameters $\left(\AA^{2}\right)$.

$\begin{array}{ccccccc} & U^{11} & U^{22} & U^{33} & U^{12} & U^{13} & U^{23} \\ \mathrm{Br} 1 & 0.0128(2) & 0.0625(3) & 0.0690(3) & 0.00183(11) & 0.00059(15) & 0.03225(17) \\ \mathrm{C} 1 & 0.0214(14) & 0.0378(15) & 0.0330(16) & 0.0050(10) & 0.0014(11) & -0.0044(11) \\ \mathrm{C} 2 & 0.0209(12) & 0.0528(18) & 0.0317(15) & 0.0089(12) & 0.0072(11) & 0.0033(13) \\ \mathrm{C} 3 & 0.0140(11) & 0.0458(16) & 0.0346(15) & 0.0014(10) & 0.0037(10) & 0.0105(12) \\ \mathrm{C} 4 & 0.0158(11) & 0.0304(13) & 0.0314(13) & -0.0019(9) & -0.0003(9) & 0.0058(11) \\ \mathrm{C} 5 & 0.0130(10) & 0.0250(12) & 0.0245(12) & 0.0024(9) & -0.0008(9) & 0.0063(9) \\ \mathrm{C} 6 & 0.0136(10) & 0.0278(12) & 0.0257(13) & 0.0019(9) & 0.0003(9) & 0.0039(10) \\ \text { C7 } & 0.0144(10) & 0.0167(10) & 0.0215(12) & -0.0003(8) & -0.0026(8) & 0.0012(9) \\ \text { C8 } & 0.0137(10) & 0.0170(10) & 0.0222(12) & 0.0005(8) & -0.0009(8) & 0.0019(9)\end{array}$




$\begin{array}{lllllll}\text { C9 } & 0.0154(11) & 0.0177(11) & 0.0312(13) & -0.0017(8) & -0.0022(9) & -0.0008(10) \\ \text { C10 } & 0.0212(12) & 0.0215(11) & 0.0280(13) & -0.0011(9) & 0.0002(10) & -0.0072(10) \\ \text { C11 } & 0.0179(11) & 0.0237(12) & 0.0233(12) & 0.0032(9) & 0.0016(9) & -0.0009(9) \\ \text { C12 } & 0.0120(10) & 0.0171(10) & 0.0216(12) & -0.0005(8) & -0.0020(8) & 0.0036(9) \\ \text { C13 } & 0.0140(10) & 0.0168(10) & 0.0187(11) & -0.0001(8) & -0.0020(8) & 0.0000(8) \\ \text { C14 } & 0.0107(10) & 0.0216(11) & 0.0226(13) & 0.0010(9) & -0.0005(9) & 0.0005(9) \\ \text { C15 } & 0.0123(10) & 0.0286(12) & 0.0261(13) & -0.0021(9) & 0.0000(9) & 0.0013(10) \\ \text { C16 } & 0.0457(17) & 0.0453(18) & 0.0509(19) & -0.0260(14) & 0.0199(15) & -0.0183(15) \\ \text { C17 } & 0.0197(12) & 0.0363(14) & 0.0337(14) & -0.0069(10) & 0.0022(10) & 0.0064(11) \\ \text { N1 } & 0.0128(9) & 0.0204(9) & 0.0273(11) & -0.0013(7) & 0.0010(7) & 0.0025(8) \\ \text { N2 } & 0.0130(9) & 0.0211(9) & 0.0185(10) & -0.0010(7) & 0.0019(8) & 0.0013(7) \\ \text { O1 } & 0.0210(9) & 0.0279(9) & 0.0477(12) & -0.0114(7) & 0.0082(8) & -0.0147(9) \\ \text { O2 } & 0.0177(9) & 0.0423(11) & 0.0202(10) & -0.0071(7) & 0.0024(7) & -0.0007(7) \\ \text { S1 } & 0.0167(3) & 0.0220(3) & 0.0292(4) & -0.00300(19) & 0.0032(3) & -0.0051(2)\end{array}$

Table S12. Geometric parameters $\left(\AA,{ }^{\circ}\right)$.

$\begin{array}{llll}\mathrm{Br} 1-\mathrm{C} 15 & 1.991(2) & \mathrm{C} 10-\mathrm{H} 10 & 0.9500 \\ \mathrm{C} 1-\mathrm{H} 1 & 0.9500 & \mathrm{C} 10-\mathrm{C} 11 & 1.380(3) \\ \mathrm{C} 1-\mathrm{C} 2 & 1.385(4) & \mathrm{C} 11-\mathrm{H} 11 & 0.9500 \\ \mathrm{C} 1-\mathrm{C} 6 & 1.395(4) & \mathrm{C} 11-\mathrm{C} 12 & 1.395(3) \\ \mathrm{C} 2-\mathrm{H} 2 & 0.9500 & \mathrm{C} 12-\mathrm{C} 13 & 1.387(3) \\ \mathrm{C} 2-\mathrm{C} 3 & 1.393(4) & \mathrm{C} 12-\mathrm{N} 2 & 1.423(3) \\ \mathrm{C} 3-\mathrm{H} 3 & 0.9500 & \mathrm{C} 13-\mathrm{H} 13 & 0.9500 \\ \mathrm{C} 3-\mathrm{C} 4 & 1.382(4) & \mathrm{C} 14-\mathrm{C} 15 & 1.540(3) \\ \mathrm{C} 4-\mathrm{H} 4 & 0.9500 & \mathrm{C} 14-\mathrm{N} 2 & 1.342(3) \\ \mathrm{C} 4-\mathrm{C} 5 & 1.400(3) & \mathrm{C} 14-\mathrm{O} 2 & 1.225(3) \\ \mathrm{C} 5-\mathrm{C} 6 & 1.401(4) & \mathrm{C} 15-\mathrm{C} 16 & 1.517(4) \\ \mathrm{C} 5-\mathrm{N} 1 & 1.388(3) & \mathrm{C} 15-\mathrm{C} 17 & 1.507(3)\end{array}$




\begin{tabular}{|c|c|c|c|}
\hline $\mathrm{C} 6-\mathrm{S} 1$ & $1.739(2)$ & $\mathrm{C} 16-\mathrm{H} 16 \mathrm{~A}$ & 0.9800 \\
\hline $\mathrm{C} 7-\mathrm{C} 8$ & $1.462(3)$ & $\mathrm{C} 16-\mathrm{H} 16 \mathrm{~B}$ & 0.9800 \\
\hline $\mathrm{C} 7-\mathrm{N} 1$ & $1.312(3)$ & $\mathrm{C} 16-\mathrm{H} 16 \mathrm{C}$ & 0.9800 \\
\hline $\mathrm{C} 7-\mathrm{S} 1$ & $1.748(2)$ & $\mathrm{C} 17-\mathrm{H} 17 \mathrm{~A}$ & 0.9800 \\
\hline $\mathrm{C} 8-\mathrm{C} 9$ & $1.409(3)$ & $\mathrm{C} 17-\mathrm{H} 17 \mathrm{~B}$ & 0.9800 \\
\hline $\mathrm{C} 8-\mathrm{C} 13$ & $1.403(3)$ & $\mathrm{C} 17-\mathrm{H} 17 \mathrm{C}$ & 0.9800 \\
\hline $\mathrm{C} 9-\mathrm{C} 10$ & $1.394(4)$ & $\mathrm{N} 2-\mathrm{H} 2 \mathrm{~A}$ & 0.8800 \\
\hline $\mathrm{C} 9-\mathrm{O} 1$ & $1.357(3)$ & $\mathrm{O} 1-\mathrm{H} 1 \mathrm{~A}$ & $0.76(4)$ \\
\hline $\mathrm{C} 2-\mathrm{C} 1-\mathrm{H} 1$ & 121.0 & $\mathrm{C} 11-\mathrm{C} 12-\mathrm{N} 2$ & $118.0(2)$ \\
\hline $\mathrm{C} 2-\mathrm{C} 1-\mathrm{C} 6$ & $118.0(3)$ & C13-C12-C11 & $119.4(2)$ \\
\hline $\mathrm{C} 6-\mathrm{C} 1-\mathrm{H} 1$ & 121.0 & $\mathrm{C} 13-\mathrm{C} 12-\mathrm{N} 2$ & $122.6(2)$ \\
\hline $\mathrm{C} 1-\mathrm{C} 2-\mathrm{H} 2$ & 119.4 & $\mathrm{C} 8-\mathrm{C} 13-\mathrm{H} 13$ & 119.6 \\
\hline $\mathrm{C} 1-\mathrm{C} 2-\mathrm{C} 3$ & $121.2(2)$ & $\mathrm{C} 12-\mathrm{C} 13-\mathrm{C} 8$ & $120.8(2)$ \\
\hline $\mathrm{C} 3-\mathrm{C} 2-\mathrm{H} 2$ & 119.4 & $\mathrm{C} 12-\mathrm{C} 13-\mathrm{H} 13$ & 119.6 \\
\hline $\mathrm{C} 2-\mathrm{C} 3-\mathrm{H} 3$ & 119.4 & $\mathrm{~N} 2-\mathrm{C} 14-\mathrm{C} 15$ & $117.0(2)$ \\
\hline $\mathrm{C} 4-\mathrm{C} 3-\mathrm{C} 2$ & $121.2(2)$ & $\mathrm{O} 2-\mathrm{C} 14-\mathrm{C} 15$ & $119.8(2)$ \\
\hline $\mathrm{C} 4-\mathrm{C} 3-\mathrm{H} 3$ & 119.4 & $\mathrm{O} 2-\mathrm{C} 14-\mathrm{N} 2$ & $123.3(2)$ \\
\hline $\mathrm{C} 3-\mathrm{C} 4-\mathrm{H} 4$ & 120.9 & $\mathrm{C} 14-\mathrm{C} 15-\mathrm{Br} 1$ & $103.78(15)$ \\
\hline $\mathrm{C} 3-\mathrm{C} 4-\mathrm{C} 5$ & $118.2(2)$ & $\mathrm{C} 16-\mathrm{C} 15-\mathrm{Br} 1$ & $107.41(19)$ \\
\hline $\mathrm{C} 5-\mathrm{C} 4-\mathrm{H} 4$ & 120.9 & C16-C15-C14 & $109.58(19)$ \\
\hline $\mathrm{C} 4-\mathrm{C} 5-\mathrm{C} 6$ & $120.4(2)$ & $\mathrm{C} 17-\mathrm{C} 15-\mathrm{Br} 1$ & $106.96(16)$ \\
\hline $\mathrm{N} 1-\mathrm{C} 5-\mathrm{C} 4$ & $125.1(2)$ & C17-C15-C14 & $117.2(2)$ \\
\hline $\mathrm{N} 1-\mathrm{C} 5-\mathrm{C} 6$ & $114.5(2)$ & $\mathrm{C} 17-\mathrm{C} 15-\mathrm{C} 16$ & $111.2(2)$ \\
\hline $\mathrm{C} 1-\mathrm{C} 6-\mathrm{C} 5$ & $121.0(2)$ & $\mathrm{C} 15-\mathrm{C} 16-\mathrm{H} 16 \mathrm{~A}$ & 109.5 \\
\hline $\mathrm{C} 1-\mathrm{C} 6-\mathrm{S} 1$ & $129.1(2)$ & $\mathrm{C} 15-\mathrm{C} 16-\mathrm{H} 16 \mathrm{~B}$ & 109.5 \\
\hline $\mathrm{C} 5-\mathrm{C} 6-\mathrm{S} 1$ & $109.87(17)$ & $\mathrm{C} 15-\mathrm{C} 16-\mathrm{H} 16 \mathrm{C}$ & 109.5 \\
\hline $\mathrm{C} 8-\mathrm{C} 7-\mathrm{S} 1$ & $122.46(16)$ & $\mathrm{H} 16 \mathrm{~A}-\mathrm{C} 16-\mathrm{H} 16 \mathrm{~B}$ & 109.5 \\
\hline
\end{tabular}




$\begin{array}{lccc}\text { N1-C7-C8 } & 122.3(2) & \mathrm{H} 16 \mathrm{~A}-\mathrm{C} 16-\mathrm{H} 16 \mathrm{C} & 109.5 \\ \mathrm{~N} 1-\mathrm{C} 7-\mathrm{S} 1 & 115.23(17) & \mathrm{H} 16 \mathrm{~B}-\mathrm{C} 16-\mathrm{H} 16 \mathrm{C} & 109.5 \\ \mathrm{C} 9-\mathrm{C} 8-\mathrm{C} 7 & 119.7(2) & \mathrm{C} 15-\mathrm{C} 17-\mathrm{H} 17 \mathrm{~A} & 109.5 \\ \mathrm{C} 13-\mathrm{C} 8-\mathrm{C} 7 & 121.2(2) & \mathrm{C} 15-\mathrm{C} 17-\mathrm{H} 17 \mathrm{~B} & 109.5 \\ \mathrm{C} 13-\mathrm{C} 8-\mathrm{C} 9 & 119.1(2) & \mathrm{C} 15-\mathrm{C} 17-\mathrm{H} 17 \mathrm{C} & 109.5 \\ \mathrm{C} 10-\mathrm{C} 9-\mathrm{C} 8 & 119.6(2) & \mathrm{H} 17 \mathrm{~A}-\mathrm{C} 17-\mathrm{H} 17 \mathrm{~B} & 109.5 \\ \mathrm{O} 1-\mathrm{C} 9-\mathrm{C} 8 & 123.0(2) & \mathrm{H} 17 \mathrm{~A}-\mathrm{C} 17-\mathrm{H} 17 \mathrm{C} & 109.5 \\ \mathrm{O} 1-\mathrm{C} 9-\mathrm{C} 10 & 117.4(2) & \mathrm{H} 17 \mathrm{~B}-\mathrm{C} 17-\mathrm{H} 17 \mathrm{C} & 109.5 \\ \mathrm{C} 9-\mathrm{C} 10-\mathrm{H} 10 & 119.8 & \mathrm{C} 7-\mathrm{N} 1-\mathrm{C} 5 & 111.3(2) \\ \mathrm{C} 11-\mathrm{C} 10-\mathrm{C} 9 & 120.5(2) & \mathrm{C} 12-\mathrm{N} 2-\mathrm{H} 2 \mathrm{~A} & 117.6 \\ \mathrm{C} 11-\mathrm{C} 10-\mathrm{H} 10 & 119.8 & \mathrm{C} 14-\mathrm{N} 2-\mathrm{C} 12 & 124.74(19) \\ \mathrm{C} 10-\mathrm{C} 11-\mathrm{H} 11 & 119.7 & \mathrm{C} 14-\mathrm{N} 2-\mathrm{H} 2 \mathrm{~A} & 117.6 \\ \mathrm{C} 10-\mathrm{C} 11-\mathrm{C} 12 & 120.6(2) & \mathrm{C} 9-\mathrm{O} 1-\mathrm{H} 1 \mathrm{~A} & 110(3) \\ \mathrm{C} 12-\mathrm{C} 11-\mathrm{H} 11 & 119.7 & \mathrm{C} 6-\mathrm{S} 1-\mathrm{C} 7 & 89.10(11) \\ & & & \end{array}$

\section{Initiator 8}

Table S13. Crystal data.

$\begin{array}{ll}\mathrm{C}_{17} \mathrm{H}_{15} \mathrm{BrN}_{2} \mathrm{O}_{3} & ? \\ M_{r}=375.22 & D_{\mathrm{x}}=1.613 \mathrm{Mg} \mathrm{m}^{-3} \\ \text { Monoclinic, } P{ }_{1} / n & \text { Melting point: ? K } \\ \text { Hall symbol: ? } & \text { Cu Ka radiation, } \lambda=1.541 \\ a=14.3934(14) \AA & \text { Cell parameters from } 5 \\ b=5.7371(5) \AA & \theta=3.8-68.6^{\circ} \\ c=18.7213(18) \AA & \mu=3.78 \mathrm{~mm}^{-1} \\ \beta=91.728(6)^{\circ} & T=150 \mathrm{~K} \\ V=1545.2(3) \AA^{3} & \text { Needle, colourless } \\ Z=4 & 0.21 \times 0.07 \times 0.05 \mathrm{~mm} \\ F(000)=760 & \end{array}$

Table S14. Data collection. 


\section{DIFFRACTOMETER \\ diffractometer}

Radiation source: ?

? monochromator

Detector resolution: ? pixels $\mathrm{mm}^{-1}$

?

Absorption correction: multi-scan

SADABS2014/5 (Bruker,2014/5) was used

for absorption correction. wR2(int) was

0.1373 before and 0.0679 after correction.

The Ratio of minimum to maximum

transmission is 0.8130 . The $N / 2$ correction

factor is 0.00150 .

$T_{\min }=0.612, T_{\max }=0.753$

$I=-22$

10183 measured reflections

2299 reflections with $I>2 \sigma(I)$

$k=-5 \quad 6$

$R_{\text {int }}=0.051$

$\theta_{\max }=68.9^{\circ}, \theta_{\min }=3.8^{\circ}$

$h=-17 \quad 14$

Table S15. Refinement.

Refinement on $F^{2}$

Least-squares matrix: full

$R\left[F^{2}>2 \sigma\left(F^{2}\right)\right]=0.075$

$w R\left(F^{2}\right)=0.222$

$S=1.05$

2807 reflections

211 parameters

0 restraints

? constraints

Primary atom site location: dual
Secondary atom site location: ?

Hydrogen site location: inferred from neighbouring sites

$\mathrm{H}$-atom parameters constrained

$w=1 /\left[\sigma^{2}\left(F_{0}^{2}\right)+(0.1235 P)^{2}+7.1699 P\right]$

where $P=\left(F_{\mathrm{o}}^{2}+2 F_{\mathrm{c}}^{2}\right) / 3$

$(\Delta / \sigma)_{\max }<0.001$

$\Delta \rho_{\max }=2.95$ e $\AA^{-3}$

$\Delta \rho_{\min }=-0.79$ e $\AA^{-3}$

Extinction correction: none

Extinction coefficient: ?

Table S16. Fractional atomic coordinates and isotropic or equivalent isotropic displacement parameters $\left(\AA^{2}\right)$.

$x$

$\mathrm{Br} 1 \quad 0.89740(5)$

C1

$\mathrm{H} 1$

C2

$\mathrm{H} 2$

0.3602 (4)

0.344356 y

1.20749 (13)

-0.0600 (10)

$-0.057536$

-0.2307 (10)

$-0.347561$ $z$

0.49988 (5)

0.8071 (3)

0.853685

0.7865 (3)

0.819934
$U_{\text {iso }}{ }^{*} / U_{\text {eq }}$

0.0536 (4)

$0.0278(12)$

$0.033^{*}$

0.0307 (13)

$0.037^{\star}$ 


\begin{tabular}{|c|c|c|c|c|}
\hline $\mathrm{C} 3$ & $0.3193(4)$ & $-0.2368(10)$ & $0.7183(3)$ & $0.0303(13)$ \\
\hline $\mathrm{H} 3$ & 0.276478 & -0.357706 & 0.706400 & $0.036^{*}$ \\
\hline $\mathrm{C} 4$ & $0.3394(4)$ & $-0.0706(9)$ & $0.6672(3)$ & $0.0271(12)$ \\
\hline $\mathrm{H} 4$ & 0.311793 & -0.073688 & 0.620487 & $0.032^{*}$ \\
\hline C5 & $0.4018(4)$ & 0.0989 (9) & $0.6886(3)$ & $0.0242(11)$ \\
\hline C6 & $0.4434(3)$ & $0.1068(9)$ & $0.7566(3)$ & $0.0233(11)$ \\
\hline $\mathrm{C} 7$ & $0.4975(3)$ & $0.3900(9)$ & $0.6948(3)$ & $0.0231(11)$ \\
\hline $\mathrm{C} 8$ & $0.5478(3)$ & $0.5909(9)$ & $0.6710(3)$ & $0.0221(11)$ \\
\hline $\mathrm{C9}$ & $0.5359(4)$ & $0.6830(9)$ & $0.6021(3)$ & $0.0254(11)$ \\
\hline H9 & 0.491999 & 0.613049 & 0.569827 & $0.030^{*}$ \\
\hline C10 & $0.5864(4)$ & $0.8728(10)$ & $0.5799(3)$ & $0.0270(12)$ \\
\hline $\mathrm{H} 10$ & 0.576292 & 0.933900 & 0.533095 & $0.032^{*}$ \\
\hline C11 & $0.6521(3)$ & $0.9746(9)$ & $0.6263(3)$ & $0.0223(11)$ \\
\hline C12 & $0.6630(4)$ & $0.8905(10)$ & $0.6966(3)$ & $0.0234(11)$ \\
\hline $\mathrm{H} 12$ & 0.705123 & 0.965527 & 0.729160 & $0.028^{*}$ \\
\hline $\mathrm{C} 13$ & $0.6130(4)$ & $0.7004(9)$ & $0.7185(3)$ & $0.0215(11)$ \\
\hline C14 & $0.7138(4)$ & $1.2724(10)$ & $0.5438(3)$ & $0.0283(12)$ \\
\hline C15 & $0.7979(4)$ & $1.4309(10)$ & $0.5313(3)$ & $0.0300(12)$ \\
\hline C16 & $0.8381(4)$ & $1.5614(10)$ & $0.5966(3)$ & $0.0316(13)$ \\
\hline $\mathrm{H} 16 \mathrm{~A}$ & 0.855601 & 1.449132 & 0.634135 & $0.047^{*}$ \\
\hline H16B & 0.791323 & 1.668972 & 0.614518 & $0.047^{*}$ \\
\hline $\mathrm{H} 16 \mathrm{C}$ & 0.893178 & 1.649567 & 0.583040 & $0.047^{*}$ \\
\hline $\mathrm{C} 17$ & $0.7760(5)$ & $1.5916(12)$ & $0.4699(3)$ & $0.0421(16)$ \\
\hline $\mathrm{H} 17 \mathrm{~A}$ & 0.829462 & 1.693070 & 0.462002 & $0.063^{*}$ \\
\hline H17B & 0.721809 & 1.687056 & 0.480947 & $0.063^{*}$ \\
\hline $\mathrm{H} 17 \mathrm{C}$ & 0.762351 & 1.499662 & 0.426763 & $0.063^{*}$ \\
\hline N1 & $0.5048(3)$ & $0.2974(7)$ & $0.7585(2)$ & $0.0238(10)$ \\
\hline N2 & $0.7138(3)$ & $1.1540(8)$ & $0.6070(2)$ & 0.0257 (10) \\
\hline $\mathrm{H} 2 \mathrm{~A}$ & 0.756727 & 1.193684 & 0.639253 & $0.031^{*}$ \\
\hline O1 & $0.4360(3)$ & $0.2814(6)$ & $0.64938(19)$ & $0.0244(8)$ \\
\hline $\mathrm{O} 2$ & $0.6276(3)$ & $0.6215(7)$ & $0.78627(19)$ & $0.0284(9)$ \\
\hline $\mathrm{H} 2 \mathrm{~B}$ & 0.595328 & 0.501784 & 0.792583 & $0.043^{*}$ \\
\hline $\mathrm{O} 3$ & $0.6519(3)$ & $1.2551(8)$ & $0.4989(2)$ & $0.0431(12)$ \\
\hline
\end{tabular}

Table S17. Atomic displacement parameters $\left(\AA^{2}\right)$. 


$\begin{array}{lcccccc} & U^{11} & U^{22} & U^{33} & U^{12} & U^{13} & U^{23} \\ \mathrm{~B} 1 & 0.0514(5) & 0.0327(5) & 0.0785(6) & 0.0000(3) & 0.0326(4) & -0.0068(3) \\ \mathrm{C} 1 & 0.023(3) & 0.026(3) & 0.034(3) & 0.000(2) & 0.006(2) & -0.001(2) \\ \mathrm{C} 2 & 0.028(3) & 0.023(3) & 0.042(3) & 0.002(2) & 0.015(3) & 0.000(2) \\ \mathrm{C} 3 & 0.024(3) & 0.021(3) & 0.046(3) & -0.002(2) & 0.012(3) & -0.010(2) \\ \mathrm{C} 4 & 0.024(3) & 0.025(3) & 0.033(3) & -0.004(2) & 0.006(2) & -0.008(2) \\ \mathrm{C} 5 & 0.021(3) & 0.020(3) & 0.032(3) & 0.001(2) & 0.008(2) & -0.007(2) \\ \mathrm{C} 6 & 0.015(2) & 0.018(3) & 0.037(3) & 0.001(2) & 0.006(2) & -0.004(2) \\ \mathrm{C} 7 & 0.016(2) & 0.020(3) & 0.034(3) & 0.004(2) & 0.001(2) & -0.007(2) \\ \mathrm{C} 8 & 0.017(2) & 0.017(2) & 0.032(3) & 0.003(2) & 0.002(2) & -0.001(2) \\ \mathrm{C} 9 & 0.022(3) & 0.027(3) & 0.026(3) & 0.001(2) & -0.001(2) & -0.002(2) \\ \mathrm{C} 10 & 0.028(3) & 0.028(3) & 0.025(3) & 0.004(2) & 0.002(2) & 0.003(2) \\ \mathrm{C} 11 & 0.020(2) & 0.022(3) & 0.025(2) & 0.003(2) & 0.004(2) & -0.003(2) \\ \mathrm{C} 12 & 0.019(2) & 0.025(3) & 0.026(3) & -0.001(2) & 0.001(2) & -0.004(2) \\ \mathrm{C} 13 & 0.018(2) & 0.021(3) & 0.026(3) & 0.006(2) & 0.003(2) & -0.003(2) \\ \mathrm{C} 14 & 0.034(3) & 0.029(3) & 0.022(3) & -0.001(2) & 0.002(2) & -0.001(2) \\ \mathrm{C} 15 & 0.036(3) & 0.023(3) & 0.032(3) & -0.001(2) & 0.007(2) & 0.000(2) \\ \mathrm{C} 16 & 0.039(3) & 0.024(3) & 0.031(3) & 0.000(2) & -0.003(2) & 0.000(2) \\ \mathrm{C} 17 & 0.058(4) & 0.037(4) & 0.031(3) & -0.002(3) & 0.003(3) & 0.007(3) \\ \mathrm{N} 1 & 0.023(2) & 0.018(2) & 0.031(2) & -0.0002(18) & 0.0014(18) & -0.0009(17) \\ \mathrm{N} 2 & 0.027(2) & 0.023(2) & 0.026(2) & -0.0034(19) & 0.0014(19) & 0.0004(18) \\ \mathrm{O} 1 & 0.026(2) & 0.0208(19) & 0.0270(18) & -0.0042(15) & 0.0028(15) & -0.0047(14) \\ \mathrm{O} 2 & 0.029(2) & 0.029(2) & 0.0267(19) & -0.0052(17) & -0.0014(16) & 0.0041(16) \\ \mathrm{O} 3 & 0.046(3) & 0.049(3) & 0.033(2) & -0.014(2) & -0.011(2) & 0.014(2)\end{array}$

Table S18. Geometric parameters $\left(\AA,{ }^{\circ}\right)$.

$\begin{array}{cccc}\mathrm{Br} 1-\mathrm{C} 15 & 2.022(6) & \mathrm{C} 10-\mathrm{H} 10 & 0.9500 \\ \mathrm{C} 1-\mathrm{H} 1 & 0.9500 & \mathrm{C} 10-\mathrm{C} 11 & 1.394(7) \\ \mathrm{C} 1-\mathrm{C} 2 & 1.383(8) & \mathrm{C} 11-\mathrm{C} 12 & 1.406(7) \\ \mathrm{C} 1-\mathrm{C} 6 & 1.382(8) & \mathrm{C} 11-\mathrm{N} 2 & 1.413(7) \\ \mathrm{C} 2-\mathrm{H} 2 & 0.9500 & \mathrm{C} 12-\mathrm{H} 12 & 0.9500 \\ \mathrm{C} 2-\mathrm{C} 3 & 1.390(9) & \mathrm{C} 12-\mathrm{C} 13 & 1.376(8) \\ \mathrm{C} 3-\mathrm{H} 3 & 0.9500 & \mathrm{C} 13-\mathrm{O} 2 & 1.357(6) \\ \mathrm{C} 3-\mathrm{C} 4 & 1.387(8) & \mathrm{C} 14-\mathrm{C} 15 & 1.538(8) \\ \mathrm{C} 4-\mathrm{H} 4 & 0.9500 & \mathrm{C} 14-\mathrm{N} 2 & 1.365(7) \\ \mathrm{C} 4-\mathrm{C} 5 & 1.375(7) & \mathrm{C} 14-\mathrm{O} 3 & 1.211(7)\end{array}$




\begin{tabular}{|c|c|c|c|}
\hline $\mathrm{C} 5-\mathrm{C} 6$ & $1.392(8)$ & C15-C16 & $1.533(8)$ \\
\hline $\mathrm{C} 5-\mathrm{O} 1$ & $1.379(7)$ & C15-C17 & $1.499(8)$ \\
\hline $\mathrm{C} 6-\mathrm{N} 1$ & $1.406(7)$ & $\mathrm{C} 16-\mathrm{H} 16 \mathrm{~A}$ & 0.9800 \\
\hline $\mathrm{C} 7-\mathrm{C} 8$ & $1.439(8)$ & $\mathrm{C} 16-\mathrm{H} 16 \mathrm{~B}$ & 0.9800 \\
\hline $\mathrm{C} 7-\mathrm{N} 1$ & $1.308(7)$ & $\mathrm{C} 16-\mathrm{H} 16 \mathrm{C}$ & 0.9800 \\
\hline $\mathrm{C} 7-\mathrm{O} 1$ & $1.359(6)$ & $\mathrm{C} 17-\mathrm{H} 17 \mathrm{~A}$ & 0.9800 \\
\hline $\mathrm{C} 8-\mathrm{C} 9$ & $1.399(8)$ & C17-H17B & 0.9800 \\
\hline $\mathrm{C} 8-\mathrm{C} 13$ & $1.420(7)$ & $\mathrm{C} 17-\mathrm{H} 17 \mathrm{C}$ & 0.9800 \\
\hline $\mathrm{C9}-\mathrm{H} 9$ & 0.9500 & $\mathrm{~N} 2-\mathrm{H} 2 \mathrm{~A}$ & 0.8800 \\
\hline $\mathrm{C} 9-\mathrm{C} 10$ & $1.380(8)$ & $\mathrm{O} 2-\mathrm{H} 2 \mathrm{~B}$ & 0.8400 \\
\hline $\mathrm{C} 2-\mathrm{C} 1-\mathrm{H} 1$ & 121.7 & $\mathrm{C} 11-\mathrm{C} 12-\mathrm{H} 12$ & 119.8 \\
\hline $\mathrm{C} 6-\mathrm{C} 1-\mathrm{H} 1$ & 121.7 & C13-C12-C11 & $120.4(5)$ \\
\hline $\mathrm{C} 6-\mathrm{C} 1-\mathrm{C} 2$ & $116.6(5)$ & $\mathrm{C} 13-\mathrm{C} 12-\mathrm{H} 12$ & 119.8 \\
\hline $\mathrm{C} 1-\mathrm{C} 2-\mathrm{H} 2$ & 119.0 & $\mathrm{C} 12-\mathrm{C} 13-\mathrm{C} 8$ & $120.4(5)$ \\
\hline $\mathrm{C} 1-\mathrm{C} 2-\mathrm{C} 3$ & $122.1(6)$ & $\mathrm{O} 2-\mathrm{C} 13-\mathrm{C} 8$ & $121.3(5)$ \\
\hline $\mathrm{C} 3-\mathrm{C} 2-\mathrm{H} 2$ & 119.0 & $\mathrm{O} 2-\mathrm{C} 13-\mathrm{C} 12$ & $118.4(5)$ \\
\hline $\mathrm{C} 2-\mathrm{C} 3-\mathrm{H} 3$ & 119.2 & $\mathrm{~N} 2-\mathrm{C} 14-\mathrm{C} 15$ & $116.6(5)$ \\
\hline $\mathrm{C} 4-\mathrm{C} 3-\mathrm{C} 2$ & $121.6(5)$ & $\mathrm{O} 3-\mathrm{C} 14-\mathrm{C} 15$ & $120.6(5)$ \\
\hline $\mathrm{C} 4-\mathrm{C} 3-\mathrm{H} 3$ & 119.2 & $\mathrm{O} 3-\mathrm{C} 14-\mathrm{N} 2$ & $122.8(5)$ \\
\hline $\mathrm{C} 3-\mathrm{C} 4-\mathrm{H} 4$ & 122.2 & $\mathrm{C} 14-\mathrm{C} 15-\mathrm{Br} 1$ & $103.7(4)$ \\
\hline $\mathrm{C} 5-\mathrm{C} 4-\mathrm{C} 3$ & $115.7(5)$ & $\mathrm{C} 16-\mathrm{C} 15-\mathrm{Br} 1$ & $106.7(4)$ \\
\hline $\mathrm{C} 5-\mathrm{C} 4-\mathrm{H} 4$ & 122.2 & C16-C15-C14 & $116.5(5)$ \\
\hline $\mathrm{C} 4-\mathrm{C} 5-\mathrm{C} 6$ & $123.3(5)$ & $\mathrm{C} 17-\mathrm{C} 15-\mathrm{Br} 1$ & $107.6(4)$ \\
\hline $\mathrm{C} 4-\mathrm{C} 5-\mathrm{O} 1$ & $128.5(5)$ & C17-C15-C14 & $109.4(5)$ \\
\hline $\mathrm{O} 1-\mathrm{C} 5-\mathrm{C} 6$ & $108.2(4)$ & C17-C15-C16 & $112.1(5)$ \\
\hline $\mathrm{C} 1-\mathrm{C} 6-\mathrm{C} 5$ & $120.7(5)$ & $\mathrm{C} 15-\mathrm{C} 16-\mathrm{H} 16 \mathrm{~A}$ & 109.5 \\
\hline $\mathrm{C} 1-\mathrm{C} 6-\mathrm{N} 1$ & $131.7(5)$ & $\mathrm{C} 15-\mathrm{C} 16-\mathrm{H} 16 \mathrm{~B}$ & 109.5 \\
\hline $\mathrm{C} 5-\mathrm{C} 6-\mathrm{N} 1$ & $107.5(5)$ & $\mathrm{C} 15-\mathrm{C} 16-\mathrm{H} 16 \mathrm{C}$ & 109.5 \\
\hline $\mathrm{N} 1-\mathrm{C} 7-\mathrm{C} 8$ & $125.5(5)$ & $\mathrm{H} 16 \mathrm{~A}-\mathrm{C} 16-\mathrm{H} 16 \mathrm{~B}$ & 109.5 \\
\hline $\mathrm{N} 1-\mathrm{C} 7-\mathrm{O} 1$ & $114.7(5)$ & $\mathrm{H} 16 \mathrm{~A}-\mathrm{C} 16-\mathrm{H} 16 \mathrm{C}$ & 109.5 \\
\hline $\mathrm{O} 1-\mathrm{C} 7-\mathrm{C} 8$ & $119.8(5)$ & $\mathrm{H} 16 \mathrm{~B}-\mathrm{C} 16-\mathrm{H} 16 \mathrm{C}$ & 109.5 \\
\hline $\mathrm{C} 9-\mathrm{C} 8-\mathrm{C} 7$ & $122.6(5)$ & $\mathrm{C} 15-\mathrm{C} 17-\mathrm{H} 17 \mathrm{~A}$ & 109.5 \\
\hline $\mathrm{C} 9-\mathrm{C} 8-\mathrm{C} 13$ & $118.1(5)$ & $\mathrm{C} 15-\mathrm{C} 17-\mathrm{H} 17 \mathrm{~B}$ & 109.5 \\
\hline $\mathrm{C} 13-\mathrm{C} 8-\mathrm{C} 7$ & $119.3(5)$ & $\mathrm{C} 15-\mathrm{C} 17-\mathrm{H} 17 \mathrm{C}$ & 109.5 \\
\hline $\mathrm{C} 8-\mathrm{C} 9-\mathrm{H} 9$ & 119.2 & $\mathrm{H} 17 \mathrm{~A}-\mathrm{C} 17-\mathrm{H} 17 \mathrm{~B}$ & 109.5 \\
\hline
\end{tabular}




$\begin{array}{cccc}\mathrm{C} 10-\mathrm{C} 9-\mathrm{C} 8 & 121.7(5) & \mathrm{H} 17 \mathrm{~A}-\mathrm{C} 17-\mathrm{H} 17 \mathrm{C} & 109.5 \\ \mathrm{C} 10-\mathrm{C} 9-\mathrm{H} 9 & 119.2 & \mathrm{H} 17 \mathrm{~B}-\mathrm{C} 17-\mathrm{H} 17 \mathrm{C} & 109.5 \\ \mathrm{C} 9-\mathrm{C} 10-\mathrm{H} 10 & 120.1 & \mathrm{C} 7-\mathrm{N} 1-\mathrm{C} 6 & 105.0(4) \\ \mathrm{C} 9-\mathrm{C} 10-\mathrm{C} 11 & 119.7(5) & \mathrm{C} 11-\mathrm{N} 2-\mathrm{H} 2 \mathrm{~A} & 116.6 \\ \mathrm{C} 11-\mathrm{C} 10-\mathrm{H} 10 & 120.1 & \mathrm{C} 14-\mathrm{N} 2-\mathrm{C} 11 & 126.9(5) \\ \mathrm{C} 10-\mathrm{C} 11-\mathrm{C} 12 & 119.6(5) & \mathrm{C} 14-\mathrm{N} 2-\mathrm{H} 2 \mathrm{~A} & 116.6 \\ \mathrm{C} 10-\mathrm{C} 11-\mathrm{N} 2 & 124.5(5) & \mathrm{C} 7-\mathrm{O} 1-\mathrm{C} 5 & 104.6(4) \\ \mathrm{C} 12-\mathrm{C} 11-\mathrm{N} 2 & 115.8(5) & \mathrm{C} 13-\mathrm{O} 2-\mathrm{H} 2 \mathrm{~B} & 109.5\end{array}$

\section{Initiator 9}

Table S19. Crystal data.

$\begin{array}{ll}\mathrm{C}_{17} \mathrm{H}_{15} \mathrm{BrN}_{2} \mathrm{O}_{3} & ? \\ M_{r}=375.22 & D_{\mathrm{x}}=1.592 \mathrm{Mg} \mathrm{m}^{-3} \\ \text { Monoclinic, } P 2_{1} / c & \text { Melting point: ? K} \\ \text { Hall symbol: ? } & \text { Mo Ka radiation, } \lambda=0.71073 \AA \\ a=14.5712(12) \AA & \text { Cell parameters from } 6911 \text { reflections } \\ b=13.290(1) \AA & \theta=2.9-26.7^{\circ} \\ c=8.3964(7) \AA & \mu=2.64 \mathrm{~mm}^{-1} \\ \beta=105.615(2)^{\circ} & T=150 \mathrm{~K} \\ V=1566.0(2) \AA^{3} & \text { Needle, colourless } \\ Z=4 & 0.38 \times 0.18 \times 0.08 \mathrm{~mm} \\ F(000)=760 & \end{array}$

Table S20. Data collection.

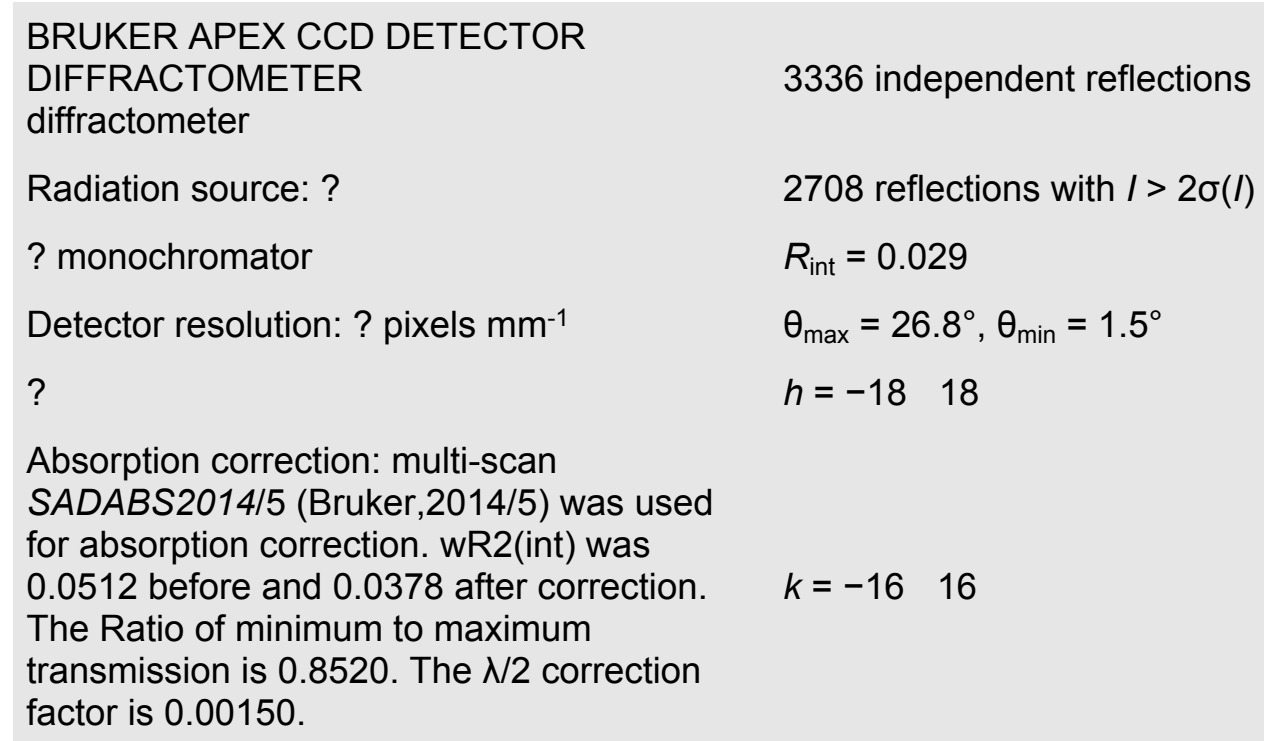

Absorption correction: multi-scan SADABS2014/5 (Bruker,2014/5) was used for absorption correction. wR2(int) was 0.0512 before and 0.0378 after correction. The Ratio of minimum to maximum transmission is 0.8520 . The $N / 2$ correction factor is 0.00150 .

3336 independent reflections

2708 reflections with $I>2 \sigma(I)$

$$
\begin{aligned}
& R_{\text {int }}=0.029 \\
& \theta_{\max }=26.8^{\circ}, \theta_{\min }=1.5^{\circ} \\
& h=-18 \quad 18
\end{aligned}
$$


$T_{\text {min }}=0.635, T_{\max }=0.745$

$I=-10 \quad 10$

17348 measured reflections

Table S21. Refinement.

$\begin{array}{ll}\text { Refinement on } F^{2} & \begin{array}{l}\text { Secondary atom site location: ? } \\ \text { Hydrogen site location: inferred from } \\ \text { neighbouring sites }\end{array} \\ R\left[F^{2}>2 \sigma\left(F^{2}\right)\right]=0.026 & \mathrm{H} \text {-atom parameters constrained } \\ w R\left(F^{2}\right)=0.070 & w=1 /\left[\sigma^{2}\left(F_{0}^{2}\right)+(0.0313 P)^{2}+1.0464 P\right] \\ & \text { where } P=\left(F_{\mathrm{o}}^{2}+2 F_{\mathrm{c}}^{2}\right) / 3 \\ S=1.06 & (\Delta / \sigma)_{\max }=0.001 \\ 3336 \text { reflections } & \Delta \rho_{\max }=0.39 \text { e } \AA^{-3} \\ 211 \text { parameters } & \Delta \rho_{\min }=-0.37 \text { e } \AA^{-3} \\ 0 \text { restraints } & \text { Extinction correction: none } \\ ? \text { constraints } & \text { Extinction coefficient: ? }\end{array}$

Primary atom site location: dual

Table S22. Fractional atomic coordinates and isotropic or equivalent isotropic displacement parameters $\left(\AA^{2}\right)$.

$\begin{array}{lcccc} & x & y & z & U_{\text {iso }}{ }^{*} U_{\text {eq }} \\ \text { Br1 } & 0.72514(2) & 0.74763(2) & 0.50744(3) & 0.02700(8) \\ \text { C1 } & -0.00681(15) & 0.65076(18) & 0.7400(3) & 0.0275(5) \\ \text { H1 } & -0.042690 & 0.707392 & 0.757501 & 0.033^{*} \\ \text { C2 } & -0.03221(16) & 0.55351(18) & 0.7696(3) & 0.0324(5) \\ \text { H2 } & -0.086694 & 0.543313 & 0.809328 & 0.039^{*} \\ \text { C3 } & 0.01949(17) & 0.47059(19) & 0.7430(3) & 0.0324(5) \\ \text { H3 } & -0.000814 & 0.405325 & 0.764765 & 0.039^{*} \\ \text { C4 } & 0.09988(17) & 0.47979(17) & 0.6855(3) & 0.0283(5) \\ \text { H4 } & 0.135385 & 0.423292 & 0.666277 & 0.034^{*} \\ \text { C5 } & 0.12427(14) & 0.57736(16) & 0.6585(2) & 0.0209(4) \\ \text { C6 } & 0.07432(14) & 0.66108(16) & 0.6832(2) & 0.0207(4) \\ \text { C7 } & 0.19373(14) & 0.71205(15) & 0.6037(2) & 0.0172(4) \\ \text { C8 } & 0.26593(14) & 0.77137(14) & 0.5573(2) & 0.0154(4) \\ \text { C9 } & 0.34544(14) & 0.72565(14) & 0.5245(2) & 0.0164(4) \\ \text { H9 } & 0.352431 & 0.654590 & 0.531687 & 0.020^{*} \\ \text { C10 } & 0.41358(14) & 0.78361(15) & 0.4819(2) & 0.0151(4) \\ & & & & \end{array}$




\begin{tabular}{ccccc} 
C11 & $0.40221(14)$ & $0.88745(14)$ & $0.4670(2)$ & $0.0173(4)$ \\
H11 & 0.448457 & 0.926923 & 0.434547 & $0.021^{*}$ \\
C12 & $0.32441(14)$ & $0.93331(15)$ & $0.4991(2)$ & $0.0194(4)$ \\
H12 & 0.317321 & 1.004258 & 0.488619 & $0.023^{*}$ \\
C13 & $0.25591(14)$ & $0.87641(15)$ & $0.5467(2)$ & $0.0170(4)$ \\
C14 & $0.55155(13)$ & $0.67265(14)$ & $0.5543(2)$ & $0.0153(4)$ \\
C15 & $0.63803(14)$ & $0.63162(14)$ & $0.5035(2)$ & $0.0177(4)$ \\
C16 & $0.60963(16)$ & $0.58869(16)$ & $0.3295(3)$ & $0.0246(5)$ \\
H16A & 0.580673 & 0.641757 & 0.251003 & $0.037^{*}$ \\
H16B & 0.563607 & 0.534093 & 0.323489 & $0.037^{*}$ \\
H16C & 0.666285 & 0.562502 & 0.301775 & $0.037^{*}$ \\
C17 & $0.69209(16)$ & $0.55635(16)$ & $0.6287(3)$ & $0.0248(5)$ \\
H17A & 0.747322 & 0.531758 & 0.594570 & $0.037^{*}$ \\
H17B & 0.650262 & 0.499692 & 0.635458 & $0.037^{*}$ \\
H17C & 0.713790 & 0.588728 & 0.737195 & $0.037^{*}$ \\
N1 & $0.12034(12)$ & $0.74683(12)$ & $0.6463(2)$ & $0.0199(3)$ \\
N2 & $0.49610(12)$ & $0.73907(11)$ & $0.4507(2)$ & $0.0171(3)$ \\
H2A & 0.511421 & 0.755764 & 0.359760 & $0.020^{*}$ \\
O1 & $0.20166(10)$ & $0.60957(10)$ & $0.60582(17)$ & $0.0195(3)$ \\
O2 & $0.18220(10)$ & $0.92533(10)$ & $0.58011(19)$ & $0.0230(3)$ \\
H2B & 0.144996 & 0.883413 & 0.604465 & $0.034^{*}$ \\
O3 & $0.53459(10)$ & $0.64263(10)$ & $0.68152(16)$ & $0.0205(3)$ \\
\hline
\end{tabular}

Table S23. Atomic displacement parameters $\left(\AA^{2}\right)$.

$\begin{array}{ccccccc} & U^{11} & U^{22} & U^{33} & U^{12} & U^{13} & U^{23} \\ \text { Br1 } & 0.01830(12) & 0.03068(13) & 0.03399(13) & -0.00180(9) & 0.01046(9) & 0.00313(10) \\ \text { C1 } & 0.0161(10) & 0.0344(12) & 0.0344(12) & -0.0006(9) & 0.0110(9) & 0.0023(10) \\ \text { C2 } & 0.0199(11) & 0.0413(14) & 0.0389(13) & -0.0089(10) & 0.0129(10) & 0.0050(11) \\ \text { C3 } & 0.0283(13) & 0.0288(12) & 0.0413(14) & -0.0115(10) & 0.0114(11) & 0.0060(10) \\ \text { C4 } & 0.0277(12) & 0.0227(11) & 0.0364(13) & -0.0048(9) & 0.0118(10) & 0.0006(10) \\ \text { C5 } & 0.0151(10) & 0.0266(11) & 0.0217(10) & -0.0035(8) & 0.0065(8) & 0.0019(9) \\ \text { C6 } & 0.0157(10) & 0.0249(11) & 0.0217(10) & -0.0032(8) & 0.0056(8) & 0.0023(8) \\ \text { C7 } & 0.0161(10) & 0.0180(9) & 0.0175(9) & -0.0002(8) & 0.0046(8) & -0.0003(8) \\ \text { C8 } & 0.0137(9) & 0.0190(10) & 0.0140(9) & -0.0015(7) & 0.0045(8) & -0.0004(7) \\ \text { C9 } & 0.0174(10) & 0.0152(9) & 0.0178(9) & 0.0018(7) & 0.0067(8) & 0.0001(7) \\ \text { C10 } & 0.0141(9) & 0.0183(9) & 0.0135(9) & 0.0033(7) & 0.0046(8) & 0.0004(7)\end{array}$




$\begin{array}{lllllll}\text { C11 } & 0.0171(10) & 0.0180(9) & 0.0182(9) & -0.0017(8) & 0.0073(8) & 0.0019(8) \\ \text { C12 } & 0.0216(11) & 0.0133(9) & 0.0242(10) & 0.0011(8) & 0.0079(9) & 0.0002(8) \\ \text { C13 } & 0.0159(10) & 0.0200(10) & 0.0161(9) & 0.0030(8) & 0.0060(8) & -0.0027(8) \\ \text { C14 } & 0.0144(9) & 0.0153(9) & 0.0172(9) & -0.0009(7) & 0.0059(8) & -0.0029(7) \\ \text { C15 } & 0.0175(10) & 0.0185(10) & 0.0191(10) & 0.0027(8) & 0.0085(8) & 0.0010(8) \\ \text { C16 } & 0.0270(12) & 0.0285(11) & 0.0201(10) & 0.0067(9) & 0.0093(9) & -0.0042(9) \\ \text { C17 } & 0.0257(12) & 0.0267(11) & 0.0230(11) & 0.0104(9) & 0.0080(9) & 0.0032(9) \\ \text { N1 } & 0.0147(8) & 0.0230(9) & 0.0237(8) & -0.0012(7) & 0.0081(7) & 0.0017(7) \\ \text { N2 } & 0.0162(8) & 0.0206(8) & 0.0179(8) & 0.0042(7) & 0.0107(7) & 0.0042(7) \\ \text { O1 } & 0.0178(7) & 0.0166(7) & 0.0269(8) & -0.0007(6) & 0.0105(6) & 0.0016(6) \\ \text { O2 } & 0.0196(8) & 0.0194(7) & 0.0342(8) & 0.0034(6) & 0.0146(7) & -0.0024(6) \\ \text { O3 } & 0.0230(8) & 0.0239(7) & 0.0178(7) & 0.0054(6) & 0.0110(6) & 0.0032(6)\end{array}$

Table S24. Geometric parameters $\left(\AA,{ }^{\circ}\right)$.

$\begin{array}{cccc}\mathrm{Br} 1-\mathrm{C} 15 & 1.992(2) & \mathrm{C} 10-\mathrm{C} 11 & 1.392(3) \\ \mathrm{C} 1-\mathrm{H} 1 & 0.9500 & \mathrm{C} 10-\mathrm{N} 2 & 1.426(2) \\ \mathrm{C} 1-\mathrm{C} 2 & 1.385(3) & \mathrm{C} 11-\mathrm{H} 11 & 0.9500 \\ \mathrm{C} 1-\mathrm{C} 6 & 1.396(3) & \mathrm{C} 11-\mathrm{C} 12 & 1.376(3) \\ \mathrm{C} 2-\mathrm{H} 2 & 0.9500 & \mathrm{C} 12-\mathrm{H} 12 & 0.9500 \\ \mathrm{C} 2-\mathrm{C} 3 & 1.387(4) & \mathrm{C} 12-\mathrm{C} 13 & 1.394(3) \\ \mathrm{C} 3-\mathrm{H} 3 & 0.9500 & \mathrm{C} 13-\mathrm{O} 2 & 1.348(2) \\ \mathrm{C} 3-\mathrm{C} 4 & 1.387(3) & \mathrm{C} 14-\mathrm{C} 15 & 1.535(3) \\ \mathrm{C} 4-\mathrm{H} 4 & 0.9500 & \mathrm{C} 14-\mathrm{N} 2 & 1.345(2) \\ \mathrm{C} 4-\mathrm{C} 5 & 1.379(3) & \mathrm{C} 14-\mathrm{O} 3 & 1.226(2) \\ \mathrm{C} 5-\mathrm{C} 6 & 1.376(3) & \mathrm{C} 15-\mathrm{C} 16 & 1.519(3) \\ \mathrm{C} 5-\mathrm{O} 1 & 1.385(2) & \mathrm{C} 15-\mathrm{C} 17 & 1.510(3) \\ \mathrm{C} 6-\mathrm{N} 1 & 1.399(3) & \mathrm{C} 16-\mathrm{H} 16 \mathrm{~A} & 0.9800 \\ \mathrm{C} 7-\mathrm{C} 8 & 1.449(3) & \mathrm{C} 16-\mathrm{H} 16 \mathrm{~B} & 0.9800 \\ \mathrm{C} 7-\mathrm{N} 1 & 1.301(3) & \mathrm{C} 16-\mathrm{H} 16 \mathrm{C} & 0.9800 \\ \mathrm{C} 7-\mathrm{O} 1 & 1.367(2) & \mathrm{C} 17-\mathrm{H} 17 \mathrm{~A} & 0.9800 \\ \mathrm{C} 8-\mathrm{C} 9 & 1.400(3) & \mathrm{C} 17-\mathrm{H} 17 \mathrm{~B} & 0.9800 \\ \mathrm{C} 8-\mathrm{C} 13 & 1.404(3) & \mathrm{C} 17-\mathrm{H} 17 \mathrm{C} & 0.9800 \\ \mathrm{C} 9-\mathrm{H} 9 & 0.9500 & \mathrm{~N} 2-\mathrm{H} 2 \mathrm{~A} & 0.8800 \\ \mathrm{C} 9-\mathrm{C} 10 & 1.378(3) & \mathrm{O} 2-\mathrm{H} 2 \mathrm{~B} & 0.8400 \\ \mathrm{C} 2-\mathrm{C} 1-\mathrm{H} 1 & 121.8 & \mathrm{C} 11-\mathrm{C} 12-\mathrm{H} 12 & 119.8 \\ \mathrm{C} 2-\mathrm{C} 1-\mathrm{C} 6 & 116.4(2) & \mathrm{C} 11-\mathrm{C} 12-\mathrm{C} 13 & 120.47(18) \\ & & & \end{array}$




\begin{tabular}{|c|c|c|c|}
\hline $\mathrm{C} 6-\mathrm{C} 1-\mathrm{H} 1$ & 121.8 & $\mathrm{C} 13-\mathrm{C} 12-\mathrm{H} 12$ & 119.8 \\
\hline $\mathrm{C} 1-\mathrm{C} 2-\mathrm{H} 2$ & 119.0 & $\mathrm{C} 12-\mathrm{C} 13-\mathrm{C} 8$ & $119.16(18)$ \\
\hline $\mathrm{C} 1-\mathrm{C} 2-\mathrm{C} 3$ & $122.0(2)$ & $\mathrm{O} 2-\mathrm{C} 13-\mathrm{C} 8$ & $122.78(18)$ \\
\hline $\mathrm{C} 3-\mathrm{C} 2-\mathrm{H} 2$ & 119.0 & $\mathrm{O} 2-\mathrm{C} 13-\mathrm{C} 12$ & $118.05(17)$ \\
\hline $\mathrm{C} 2-\mathrm{C} 3-\mathrm{H} 3$ & 118.9 & $\mathrm{~N} 2-\mathrm{C} 14-\mathrm{C} 15$ & $116.64(16)$ \\
\hline $\mathrm{C} 2-\mathrm{C} 3-\mathrm{C} 4$ & $122.2(2)$ & $\mathrm{O} 3-\mathrm{C} 14-\mathrm{C} 15$ & $119.96(17)$ \\
\hline $\mathrm{C} 4-\mathrm{C} 3-\mathrm{H} 3$ & 118.9 & $\mathrm{O} 3-\mathrm{C} 14-\mathrm{N} 2$ & $123.37(17)$ \\
\hline $\mathrm{C} 3-\mathrm{C} 4-\mathrm{H} 4$ & 122.6 & $\mathrm{C} 14-\mathrm{C} 15-\mathrm{Br} 1$ & $106.72(12)$ \\
\hline $\mathrm{C} 5-\mathrm{C} 4-\mathrm{C} 3$ & $114.8(2)$ & $\mathrm{C} 16-\mathrm{C} 15-\mathrm{Br} 1$ & $108.30(13)$ \\
\hline $\mathrm{C} 5-\mathrm{C} 4-\mathrm{H} 4$ & 122.6 & C16-C15-C14 & $111.67(17)$ \\
\hline $\mathrm{C} 4-\mathrm{C} 5-\mathrm{O} 1$ & 127.65 (19) & $\mathrm{C} 17-\mathrm{C} 15-\mathrm{Br} 1$ & $106.89(14)$ \\
\hline $\mathrm{C} 6-\mathrm{C} 5-\mathrm{C} 4$ & $124.44(19)$ & C17-C15-C14 & $110.83(16)$ \\
\hline $\mathrm{C} 6-\mathrm{C} 5-\mathrm{O} 1$ & $107.90(17)$ & $\mathrm{C} 17-\mathrm{C} 15-\mathrm{C} 16$ & $112.12(17)$ \\
\hline $\mathrm{C} 1-\mathrm{C} 6-\mathrm{N} 1$ & $131.04(19)$ & $\mathrm{C} 15-\mathrm{C} 16-\mathrm{H} 16 \mathrm{~A}$ & 109.5 \\
\hline $\mathrm{C} 5-\mathrm{C} 6-\mathrm{C} 1$ & $120.22(19)$ & $\mathrm{C} 15-\mathrm{C} 16-\mathrm{H} 16 \mathrm{~B}$ & 109.5 \\
\hline $\mathrm{C} 5-\mathrm{C} 6-\mathrm{N} 1$ & $108.72(17)$ & $\mathrm{C} 15-\mathrm{C} 16-\mathrm{H} 16 \mathrm{C}$ & 109.5 \\
\hline $\mathrm{N} 1-\mathrm{C} 7-\mathrm{C} 8$ & $126.21(18)$ & $\mathrm{H} 16 \mathrm{~A}-\mathrm{C} 16-\mathrm{H} 16 \mathrm{~B}$ & 109.5 \\
\hline $\mathrm{N} 1-\mathrm{C} 7-\mathrm{O} 1$ & $115.06(17)$ & $\mathrm{H} 16 \mathrm{~A}-\mathrm{C} 16-\mathrm{H} 16 \mathrm{C}$ & 109.5 \\
\hline $\mathrm{O} 1-\mathrm{C} 7-\mathrm{C} 8$ & $118.73(17)$ & $\mathrm{H} 16 \mathrm{~B}-\mathrm{C} 16-\mathrm{H} 16 \mathrm{C}$ & 109.5 \\
\hline $\mathrm{C} 9-\mathrm{C} 8-\mathrm{C} 7$ & $121.05(17)$ & $\mathrm{C} 15-\mathrm{C} 17-\mathrm{H} 17 \mathrm{~A}$ & 109.5 \\
\hline $\mathrm{C} 9-\mathrm{C} 8-\mathrm{C} 13$ & $119.78(18)$ & C15-C17-H17B & 109.5 \\
\hline $\mathrm{C} 13-\mathrm{C} 8-\mathrm{C} 7$ & $119.16(18)$ & $\mathrm{C} 15-\mathrm{C} 17-\mathrm{H} 17 \mathrm{C}$ & 109.5 \\
\hline $\mathrm{C} 8-\mathrm{C} 9-\mathrm{H} 9$ & 120.0 & $\mathrm{H} 17 \mathrm{~A}-\mathrm{C} 17-\mathrm{H} 17 \mathrm{~B}$ & 109.5 \\
\hline $\mathrm{C} 10-\mathrm{C} 9-\mathrm{C} 8$ & $120.05(18)$ & $\mathrm{H} 17 \mathrm{~A}-\mathrm{C} 17-\mathrm{H} 17 \mathrm{C}$ & 109.5 \\
\hline C10-C9-H9 & 120.0 & $\mathrm{H} 17 \mathrm{~B}-\mathrm{C} 17-\mathrm{H} 17 \mathrm{C}$ & 109.5 \\
\hline $\mathrm{C} 9-\mathrm{C} 10-\mathrm{C} 11$ & $120.08(18)$ & $\mathrm{C} 7-\mathrm{N} 1-\mathrm{C} 6$ & $104.49(16)$ \\
\hline $\mathrm{C} 9-\mathrm{C} 10-\mathrm{N} 2$ & $121.18(17)$ & $\mathrm{C} 10-\mathrm{N} 2-\mathrm{H} 2 \mathrm{~A}$ & 118.6 \\
\hline $\mathrm{C} 11-\mathrm{C} 10-\mathrm{N} 2$ & $118.74(17)$ & $\mathrm{C} 14-\mathrm{N} 2-\mathrm{C} 10$ & $122.87(15)$ \\
\hline C10-C11-H11 & 119.8 & $\mathrm{C} 14-\mathrm{N} 2-\mathrm{H} 2 \mathrm{~A}$ & 118.6 \\
\hline C12-C11-C10 & $120.40(18)$ & $\mathrm{C} 7-\mathrm{O} 1-\mathrm{C} 5$ & $103.82(15)$ \\
\hline $\mathrm{C} 12-\mathrm{C} 11-\mathrm{H} 11$ & 119.8 & $\mathrm{C} 13-\mathrm{O} 2-\mathrm{H} 2 \mathrm{~B}$ & 109.5 \\
\hline
\end{tabular}

All e.s.d.'s (except the e.s.d. in the dihedral angle between two I.s. planes) are estimated using the full covariance matrix. The cell e.s.d.'s are taken into account individually in the estimation of e.s.d.'s in distances, angles and torsion angles; correlations between e.s.d.'s in cell parameters are only used when they 
are defined by crystal symmetry. An approximate (isotropic) treatment of cell e.s.d.'s is used for estimating e.s.d.'s involving I.s. planes
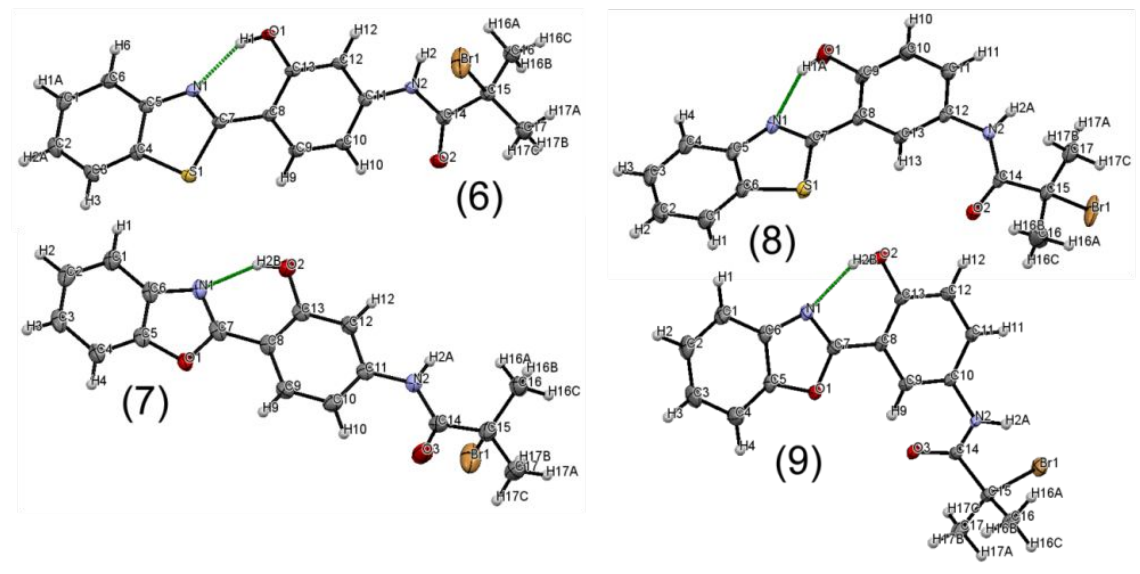

Figure S29. Molecular structure of ATRP initiators 6-9 with thermal ellipsoids at the $50 \%$ probability level.

Table S25. Hydrogen Bond Geometry by X-Ray Diffraction (XRD).

\begin{tabular}{lllll}
\hline $\mathbf{0}-\mathbf{H} \cdots \mathbf{N}$ & $\mathbf{0}-\mathbf{H}(\AA)$ & $\mathbf{H} \cdots \mathbf{N}(\AA)$ & $\mathbf{D} \cdots \mathbf{N}(\AA)$ & $\mathbf{0}-\mathbf{H} \cdots \mathbf{N}\left({ }^{\circ}\right)$ \\
\hline $\mathbf{( 6 )}$ & 0.840 & 1.850 & 2.603 & 148.48 \\
$\mathbf{( 7 )}$ & 0.840 & 1.852 & 2.607 & 148.87 \\
$\mathbf{( 8 )}$ & 0.764 & 1.938 & 2.601 & 145.63 \\
$\mathbf{( 9 )}$ & 0.840 & 1.901 & 2.649 & 147.61 \\
\hline
\end{tabular}

Thermal Characterization 

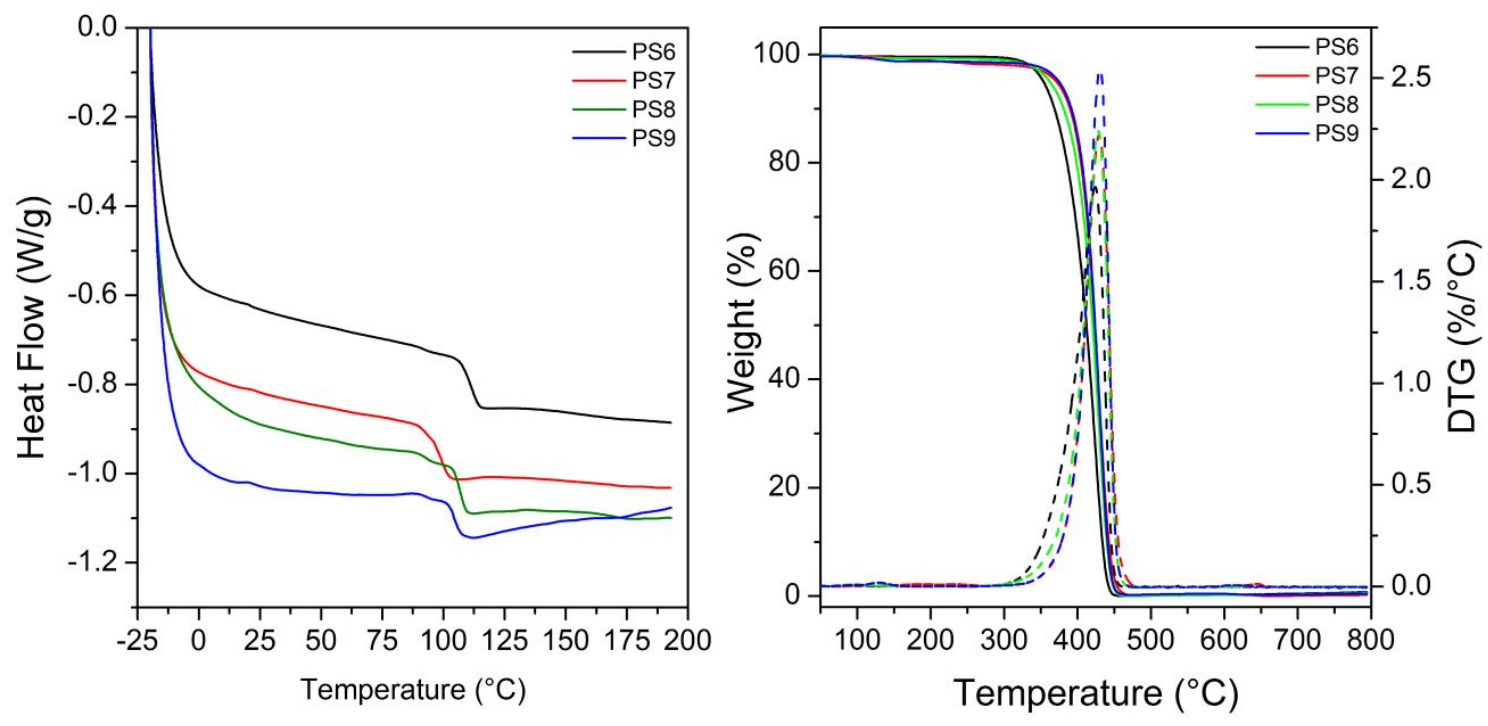

Figure S30. DSC (left) and TGA/DTG thermograms (right) of polymers PS6-9.

Table S26. Polymerization data and thermal properties of the polymers PS6-9, where $\bar{M}_{\mathrm{w}} \times 10^{3}\left(\mathrm{~g} \cdot \mathrm{mol}^{-1}\right)$ and $\bar{M}_{\mathrm{n}} \times 10^{3}\left(\mathrm{~g} \cdot \mathrm{mol}^{-1}\right)$ are the weight average molecular weight and the number average molecular weight, respectively obtained by Size Exclusion Chromatography (SEC), $T_{g}$ is the glass transition temperature $\left({ }^{\circ} \mathrm{C}\right), \mathrm{T}_{\mathrm{di}}$ and $\mathrm{T}_{\mathrm{df}}$ are the initial and final decomposition temperatures $\left({ }^{\circ} \mathrm{C}\right)$ and $\mathrm{T}_{\mathrm{d}}$ is the decomposition temperature $\left({ }^{\circ} \mathrm{C}\right)$ at the maximum DTG curve.

\begin{tabular}{ccccc}
\hline & PS6 & PS7 & PS8 & PS9 \\
\hline Yield (\%) & 84 & 80 & 82 & 78 \\
$\overline{\boldsymbol{M}} \boldsymbol{w}$ & 104.7 & 94.7 & 85.0 & 84.5 \\
$\overline{\boldsymbol{M}} \boldsymbol{n}$ & 86.5 & 71.4 & 62.5 & 60.7 \\
Polydispersity & 1.211 & 1.326 & 1.361 & 1.393 \\
$\mathbf{T}_{\mathbf{g}} \mathbf{a}$ & 111 & 99 & 106 & 104 \\
$\mathbf{T}_{\mathbf{d i}} \mathbf{b}$ & 365 & 396 & 394 & 396 \\
$\mathbf{T}_{\mathbf{d f}} \mathbf{a}$ & 448 & 452 & 450 & 450 \\
$\mathbf{T}_{\mathbf{d}} \mathbf{b}$ & 424 & 429 & 429 & 429 \\
\hline
\end{tabular}

${ }^{a}$ Glass transition temperature measured using DSC.

${ }^{\mathrm{b} T e m p e r a t u r e ~ o f ~ d e c o m p o s i t i o n ~ o b t a i n e d ~ b y ~ T G A ~ i n ~ a t m o s p h e r e ~ o f ~} \mathrm{~N}_{2}$ at $10^{\circ} \mathrm{C} \cdot \mathrm{min}^{-1}$. 


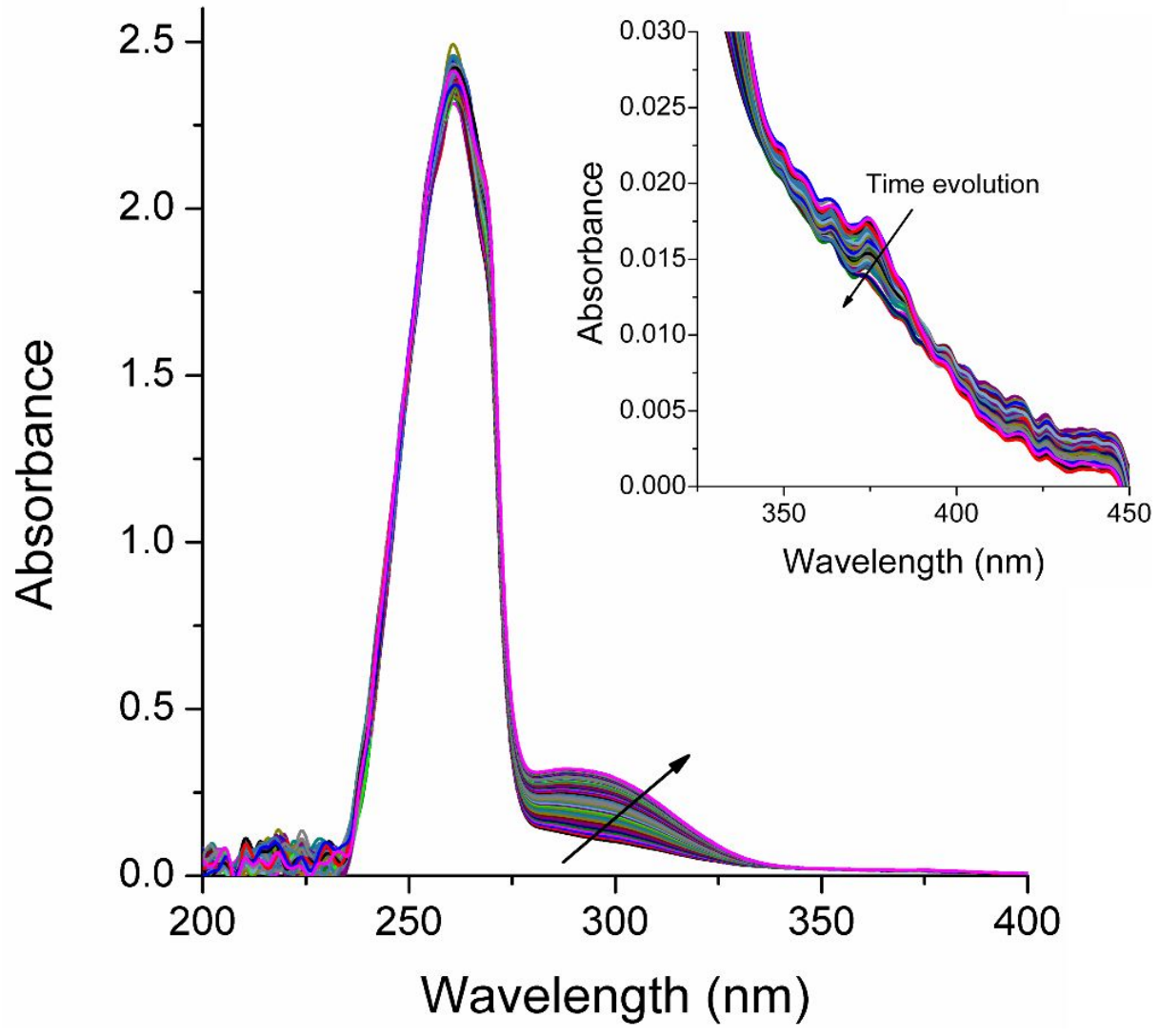

Figure S31. Photostability test of PS6 in THF solution (4 mg mL-1) using an 200 W Hg/Xe lamp and acquiring the UV-Vis absorption spectra every 2 min up to $50 \mathrm{~min}$. The inset indicates the magnification of the region due to the respective initiator. 


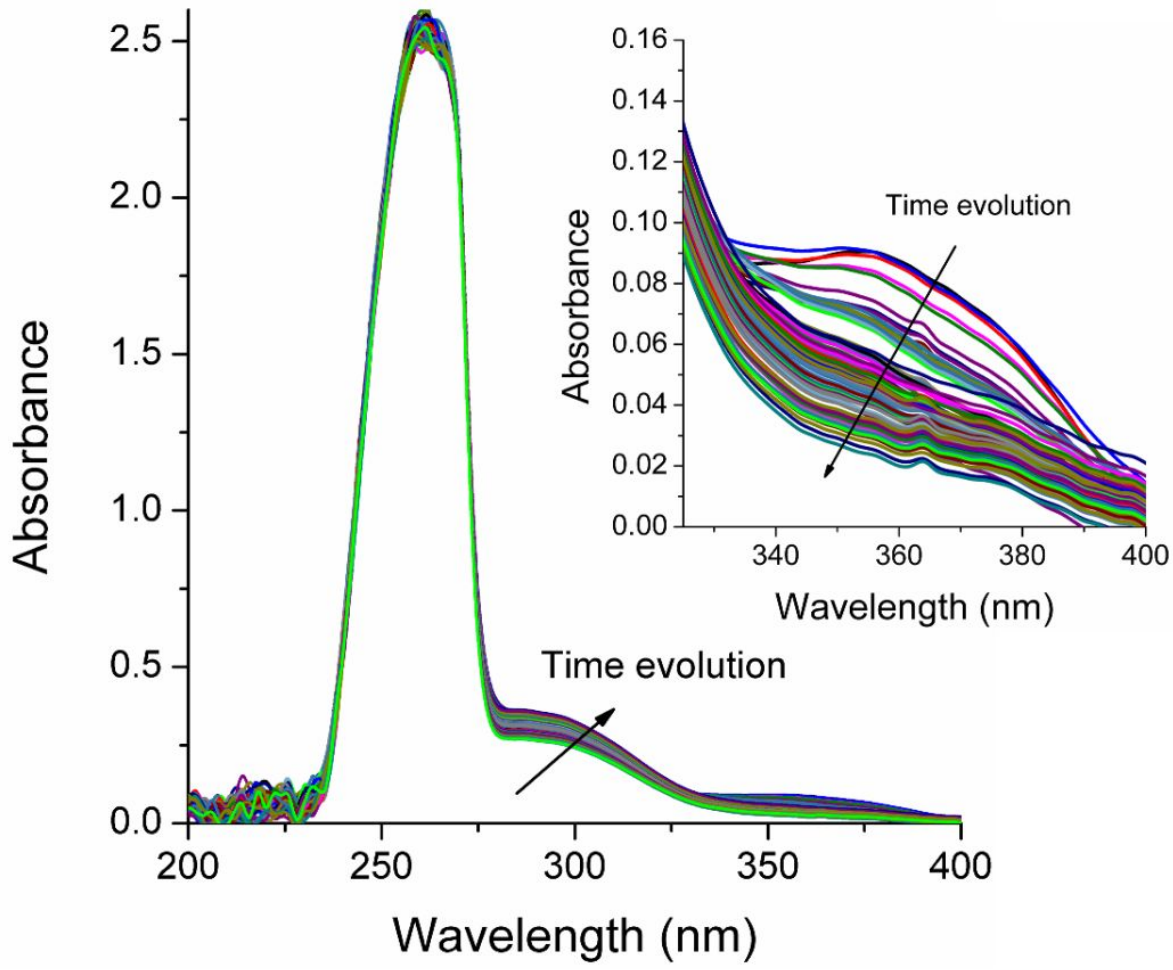

Figure S32. Photostability test of PS8 in THF solution (4 mg mL-1) using an 200 W Hg/Xe lamp and acquiring the UV-Vis absorption spectra every 2 min up to $50 \mathrm{~min}$. The inset indicates the magnification of the region due to the respective initiator.

Table S27. Photodegradation data for the polymers PS6-9 in THF solution (4 mg mL-1) using an $200 \mathrm{~W} \mathrm{Hg} / \mathrm{Xe}$ lamp at a total time of 50 $\min$.

\begin{tabular}{c|c|c|c|c}
\hline \multirow{2}{*}{ Sample } & \multicolumn{2}{|c|}{ Curve Slope } & \multicolumn{2}{c}{ Rate } \\
\cline { 2 - 5 } & $\lambda_{\text {abs }}=\mathbf{3 0 0} \mathrm{nm}$ & $\lambda_{\text {abs }}$ dye & $\lambda_{\text {abs }}=\mathbf{3 0 0 ~} \mathrm{nm}$ & $\lambda_{\text {abs }}$ dye \\
\hline PS & 0.17 & $*$ & 5.76 & $*$ \\
PS6 & 0.10 & -0.32 & 9.88 & 3.11 \\
PS8 & 0.05 & -0.21 & 19.11 & 4.67 \\
PS7 & 0.10 & -0.63 & 9.70 & 1.58 \\
PS9 & 0.07 & -0.17 & 14.44 & 5.77 \\
\hline
\end{tabular}




\section{Additional photophysical data}

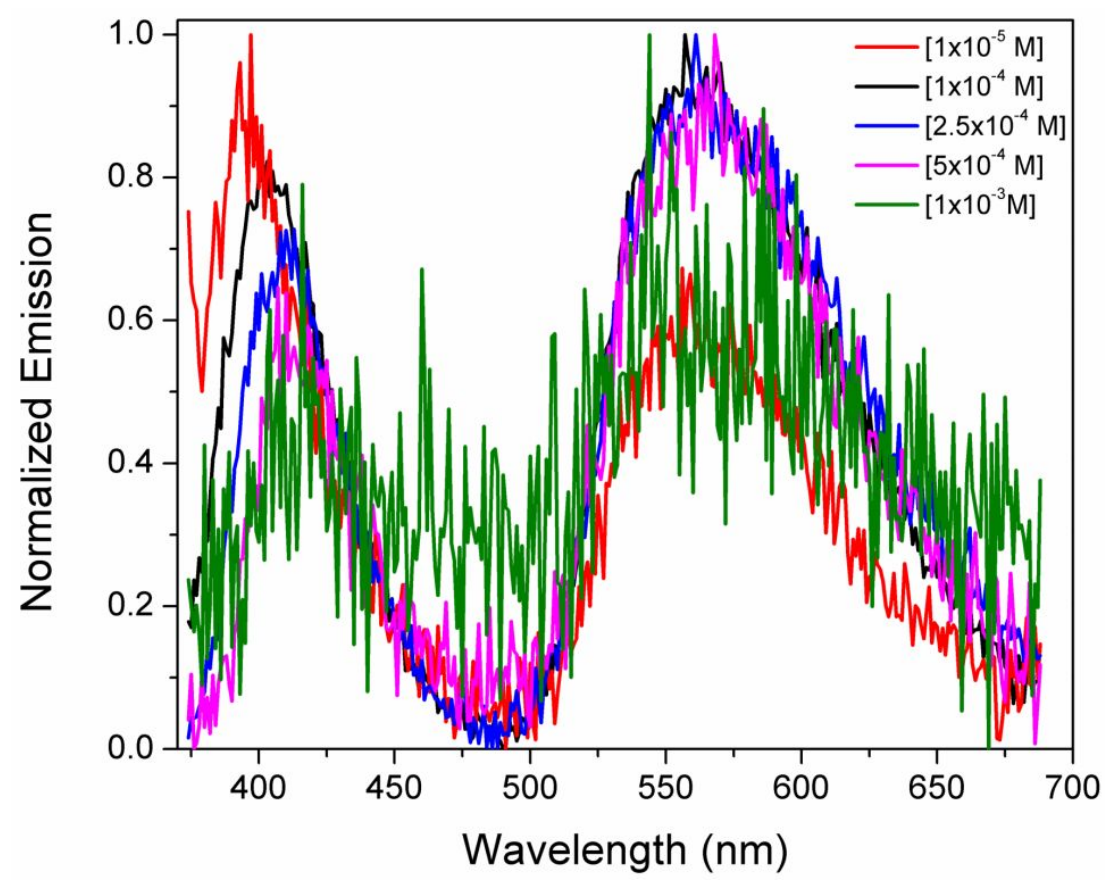

Figure S33. Normalized steady-state fluorescence emission spectra of initiator 8 at different solution concentrations.

\section{Data treatment of time resolved fluorescence}

The fluorescence decay curves were acquired using 10,000 counts at the peak channel and the samples were excited using an intense and stable excitation source (a pulse diode). The equation applied to adjust the decays is a sum of exponentials (Eq. 1), where $I(t)$ is the count intensity of the electronic excited species at time $=t$ after the excitation pulse weighted by $A_{i}$ (the preexponential factor) and with a decay constant $\tau_{i}$ for decay component $i$. Eq. 1 is only valid because the excitation source signal is firstly deconvoluted from the overall data using the software FAST.

$$
I(t)=\sum_{i=1}^{n} A_{i} e^{-\frac{t}{\tau_{i}}} \quad(\text { Eq. 1) }
$$


Additionally, to remove the signal from excitation source and determine the lifetimes with valid statistical meaning, the decay curves were adjusted accounting for as many channels as possible, initiating the adjusts before the decays peak channel where the curves start to rise around 10 counts, until the decays reach $\sim 10$ counts again. This is a valid strategy to avoid scattering and additional sort of noise, once the decays are in general very short and close to the instrument response function (IRF). It is worth mentioning that an optical filter to block the light until $350 \mathrm{~nm}$ was added to the set-up to avoid contribution from excitation source when the decays were collected. A final strategy to avoid scattering was to increase the time-window, so the accumulation time/channel diminishes - obviously, each window required a new IRF to the fitting procedure. 


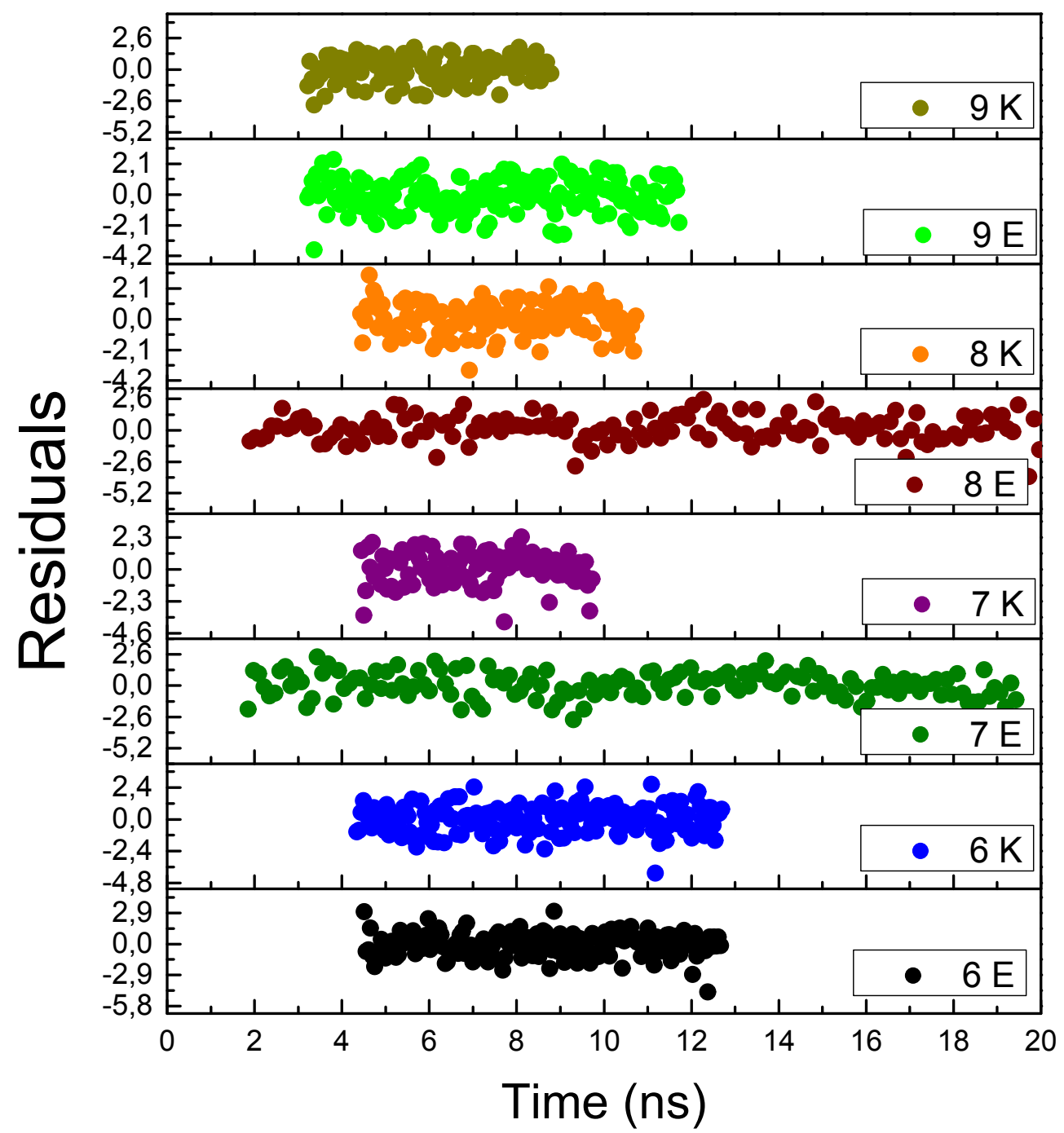

Figure S34. Residuals for the time decay curves of monomers 6-9. $E$ and $K$ are related to the emissions from the enol conformer (normal emission) and keto tautomer (ESIPT emission), respectively. 


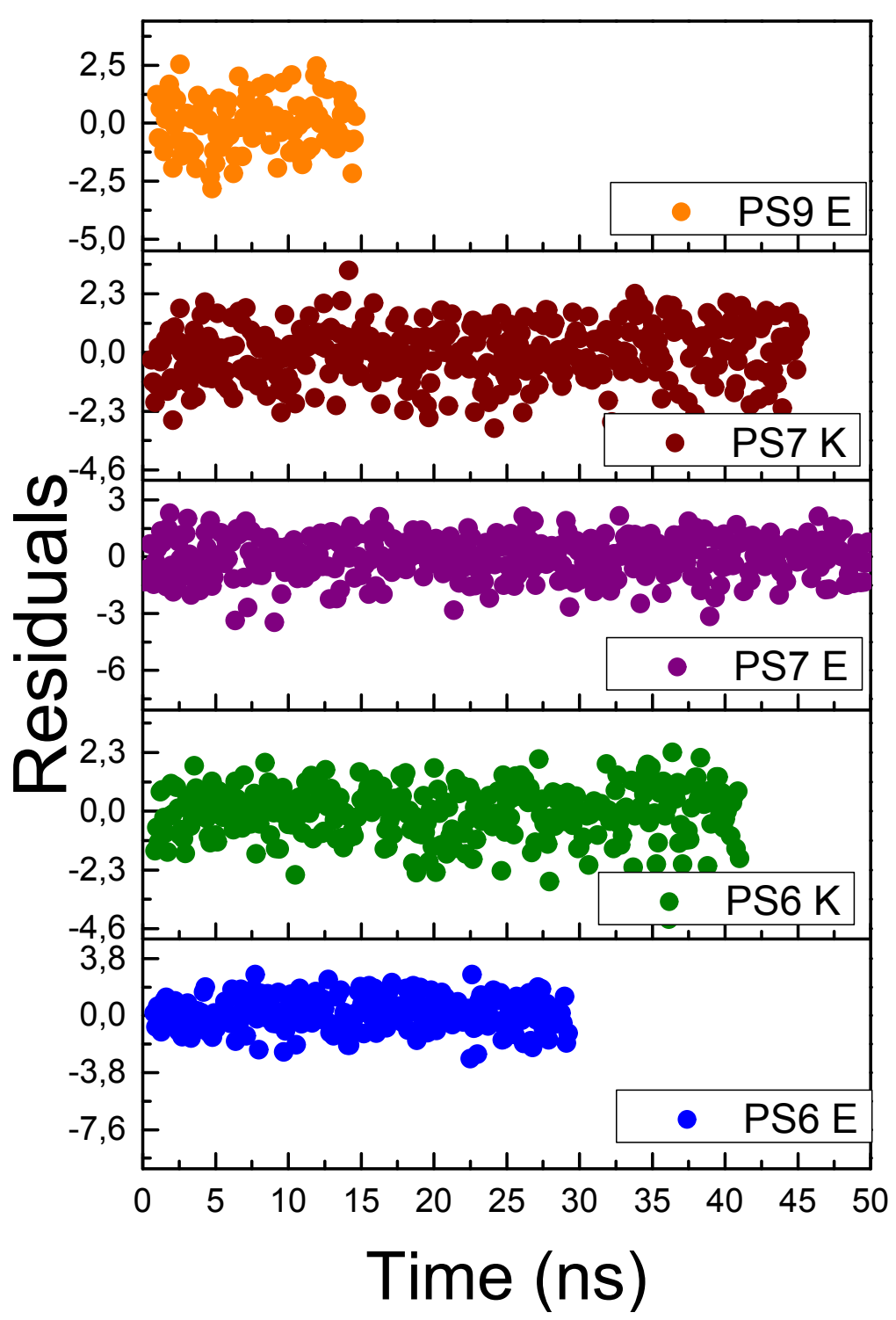

Figure S35. Residuals for the time decay curves of polymers PS6, PS 7 and PS9. $\mathrm{E}$ and $\mathrm{K}$ are related to the emissions from the enol conformer (normal emission) and keto tautomer (ESIPT emission), respectively. 

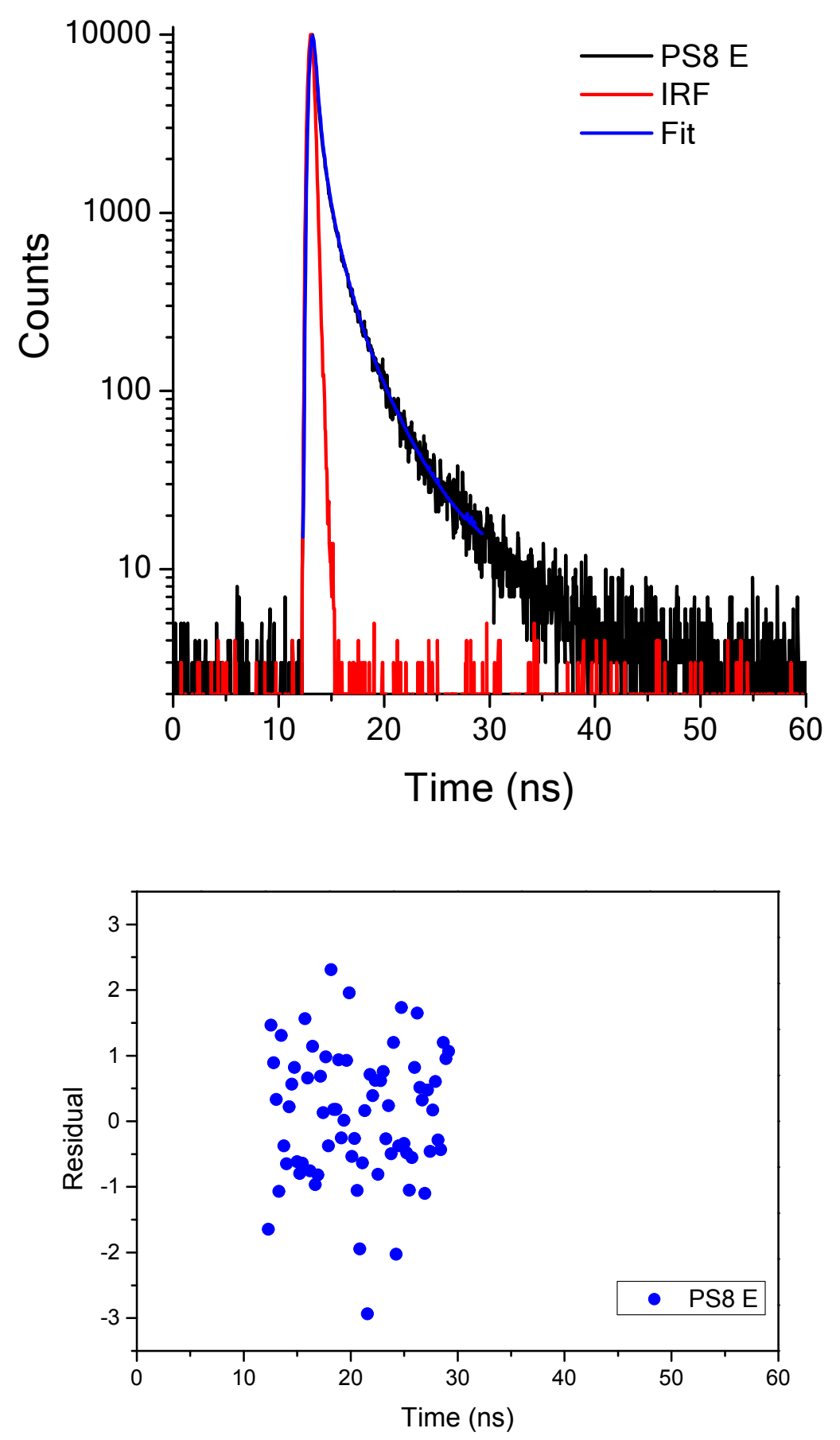

Figure S36. Fluorescence decay (top) and respective exponential fit (bottom) for the PS8 in THF solution at emission maxima of $385 \mathrm{~nm}$ (E emission) and excitation at $335 \mathrm{~nm}$ (top). $E$ is related to the emissions from the enol conformer (normal emission). 

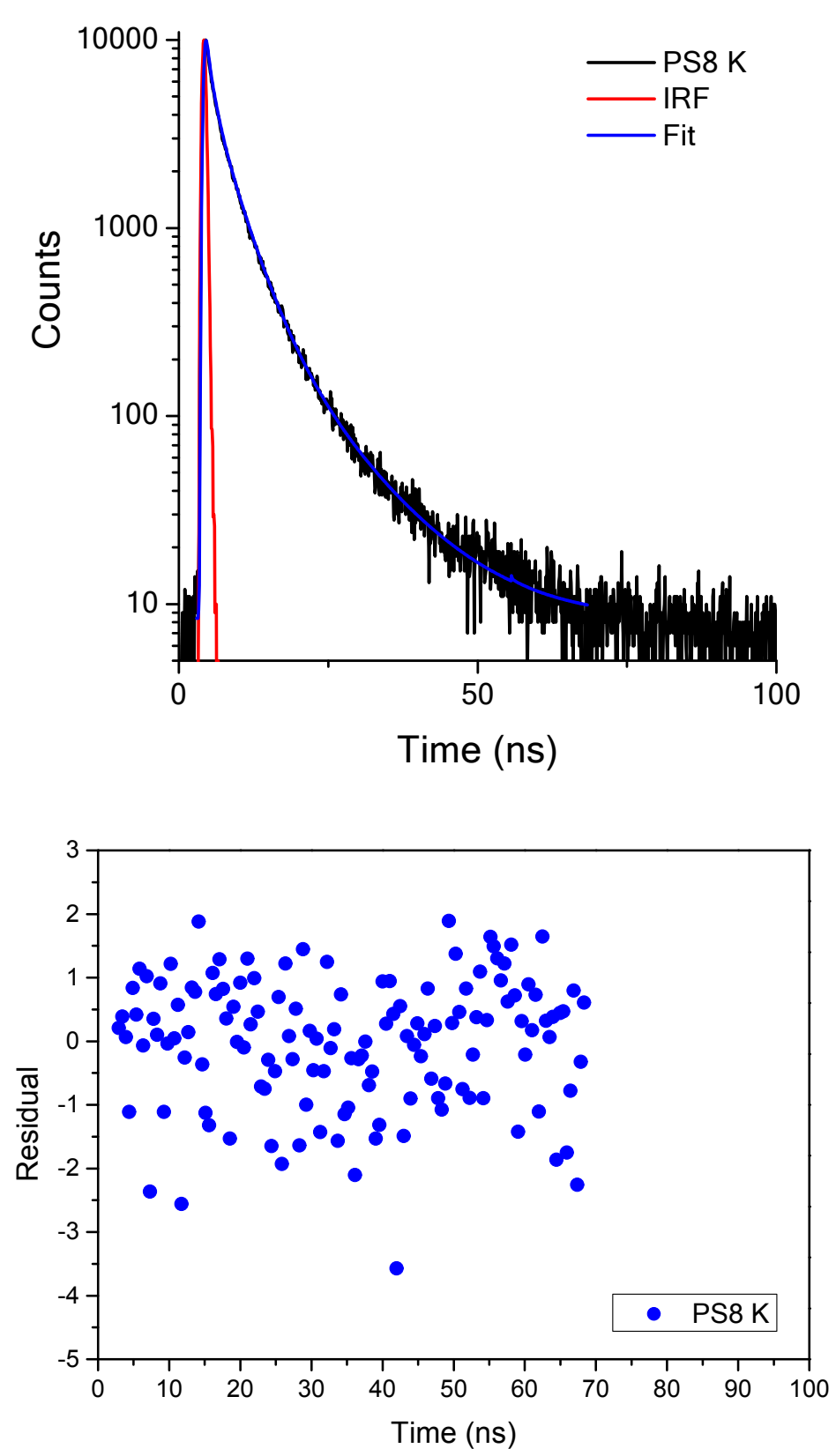

Figure S37. Fluorescence decay (top) and respective exponential fit (bottom) for the PS8 in THF solution at emission maxima of $485 \mathrm{~nm}$ (K emission) and excitation at $335 \mathrm{~nm}$ (top). $\mathrm{K}$ is related to the emissions from the enol conformer (normal emission). 

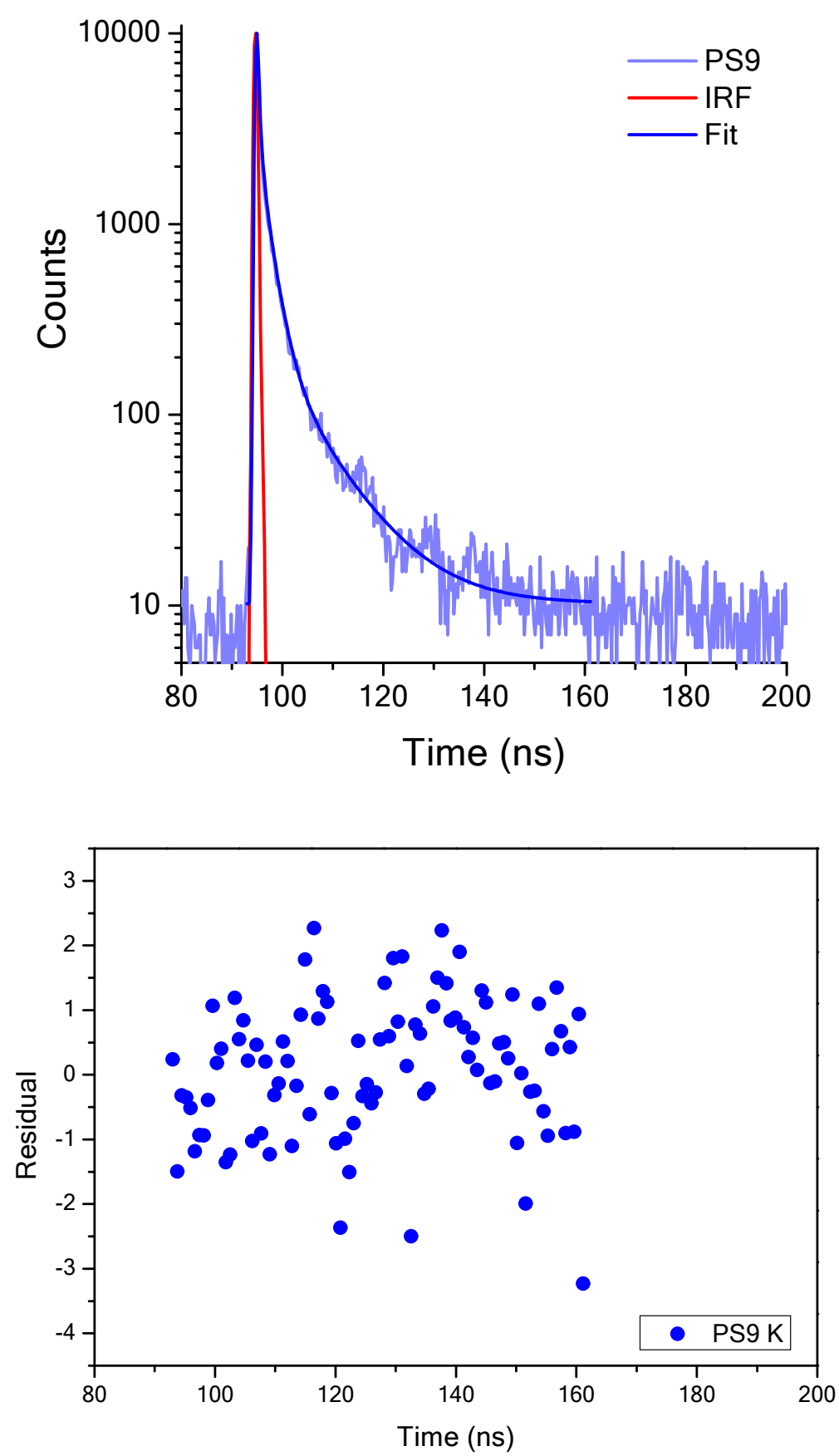

Figure S38. Fluorescence decay (top) and respective exponential fit (bottom) for the PS9 in THF solution at emission maxima of $520 \mathrm{~nm}$ (K emission) and excitation at $335 \mathrm{~nm}$ (top). $\mathrm{K}$ is related to the emissions from the enol conformer (normal emission). 
Table S28. Fluorescence lifetimes for ATRP initiators 6-9 and respective polymers PS6-9 in THF solutions, where $\tau_{\mathrm{E}}$ and $\tau_{\mathrm{K}}$ are the $\mathrm{E}^{*}$ and $\mathrm{K}^{*}$ lifetimes, respectively at $\lambda_{\mathrm{exc}}=335 \mathrm{~nm}, \lambda_{\mathrm{em}}$ is the emission maxima in nanometers and $A_{i}$ is the pre-exponential factor. Decays were collected at emissions peaks.

\begin{tabular}{ccccccc}
\hline Sample & $\lambda_{\text {em }}$ & $\mathbf{T}_{E}$ & $A_{E}$ & $\mathbf{T}_{\mathbf{K}}$ & $\boldsymbol{A}_{K}$ & $\mathbf{X}^{\mathbf{2}}$ \\
\hline \multirow{2}{*}{$\mathbf{6}$} & 396 & $2.22 \pm 0.02$ & 10 & $0.65 \pm 0.01$ & 90 & 1.128 \\
& 511 & $2.57 \pm 0.02$ & 20 & $0.60 \pm 0.05$ & 80 & 1.124 \\
\hline \multirow{2}{*}{$\mathbf{7}$} & 370 & $1.27 \pm 0.05$ & 25 & $0.46 \pm 0.08$ & 75 & 1.037 \\
& 480 & $1.21 \pm 0.15$ & 10 & $0.30 \pm 0.17$ & 90 & 1.111 \\
\hline \multirow{2}{*}{$\mathbf{8}$} & 396 & $1.45 \pm 0.01$ & 80 & $0.55 \pm 0.16$ & 20 & 1.078 \\
& 555 & $1.88 \pm 0.04$ & 40 & $0.45 \pm 0.13$ & 60 & 1.293 \\
\hline \multirow{2}{*}{$\mathbf{9}$} & 378 & $3.94 \pm 0.02$ & 12 & $0.50 \pm 0.03$ & 88 & 1.135 \\
& 518 & $\sim$ FWHM & & $0.50 \pm 0.20$ & 100 & 1.063 \\
\hline \multirow{2}{*}{ PS6 } & 397 & $2.72 \pm 0.03$ & 75 & $0.90 \pm 0.04$ & 25 & 1.181 \\
& 492 & $3.00 \pm 0.02$ & 63 & $1.12 \pm 0.07$ & 27 & 1.057 \\
\hline \multirow{2}{*}{ PS7 } & 415 & $2.91 \pm 0.02$ & 48 & $1.16 \pm 0.03$ & 52 & 1.169 \\
& 471 & $3.18 \pm 0.01$ & 70 & $1.02 \pm 0.05$ & 30 & 1.055 \\
\hline \multirow{2}{*}{ PS8 } & 385 & $3.40 \pm 0.03$ & 55 & $1.18 \pm 0.10$ & 45 & 1.093 \\
& 485 & $3.74 \pm 0.01$ & 77 & $1.05 \pm 0.01$ & 23 & 1.068 \\
\hline \multirow{2}{*}{ PS9 } & 400 & $1.64 \pm 0.05$ & 80 & $0.76 \pm 0.06$ & 20 & 1.129 \\
& 520 & $2.10 \pm 0.03$ & 70 & $0.62 \pm 0.50$ & 30 & 1.309 \\
\hline
\end{tabular}

Table S29. Photophysical data of the initiators 6-9 and respective polymers PS6-9 in the solid state, where $\lambda_{\text {abs }}$ is the absorption maxima $(\mathrm{nm}), \lambda_{\text {em }}$ is the emission maxima $(\mathrm{nm}), \Delta \lambda_{\mathrm{ST}}$ is the Stokes shift $\left(\mathrm{nm} / \mathrm{cm}^{-1}\right)$, and $\mathrm{QY}$ is the relative quantum yield of fluorescence of the ESIPT compounds (\%).

\begin{tabular}{|c|c|c|c|c|c|c|}
\hline \multirow{2}{*}{ Sample } & \multirow{2}{*}{$\lambda_{\text {abs }}$} & \multicolumn{2}{|c|}{ Enol $^{*}\left(E^{*}\right)$} & \multicolumn{2}{|c|}{ Keto* $\left(K^{*}\right)$} & \multirow{2}{*}{$Q Y$} \\
\hline & & $\lambda_{\mathrm{em}}$ & $\Delta \lambda_{\mathrm{ST}}$ & $\lambda_{\mathrm{em}}$ & $\Delta \lambda_{\mathrm{ST}}$ & \\
\hline 6 & 365 & 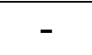 & 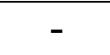 & 500 & $135 / 7397$ & 1.9 \\
\hline 7 & 330 & - & - & 462 & $132 / 8658$ & 1.8 \\
\hline 8 & 354 & - & - & 550 & $196 / 10067$ & 2.1 \\
\hline 9 & 340 & - & - & 463 & $123 / 7814$ & 3.4 \\
\hline PS6 & 359 & - & - & 511 & $152 / 8286$ & 10.0 \\
\hline PS7 & 352 & - & - & 462 & $110 / 6764$ & 34.4 \\
\hline PS8 & 363 & 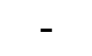 & - & 548 & $185 / 9300$ & 4.4 \\
\hline PS9 & 360 & - & - & 511 & $151 / 8208$ & 7.8 \\
\hline
\end{tabular}



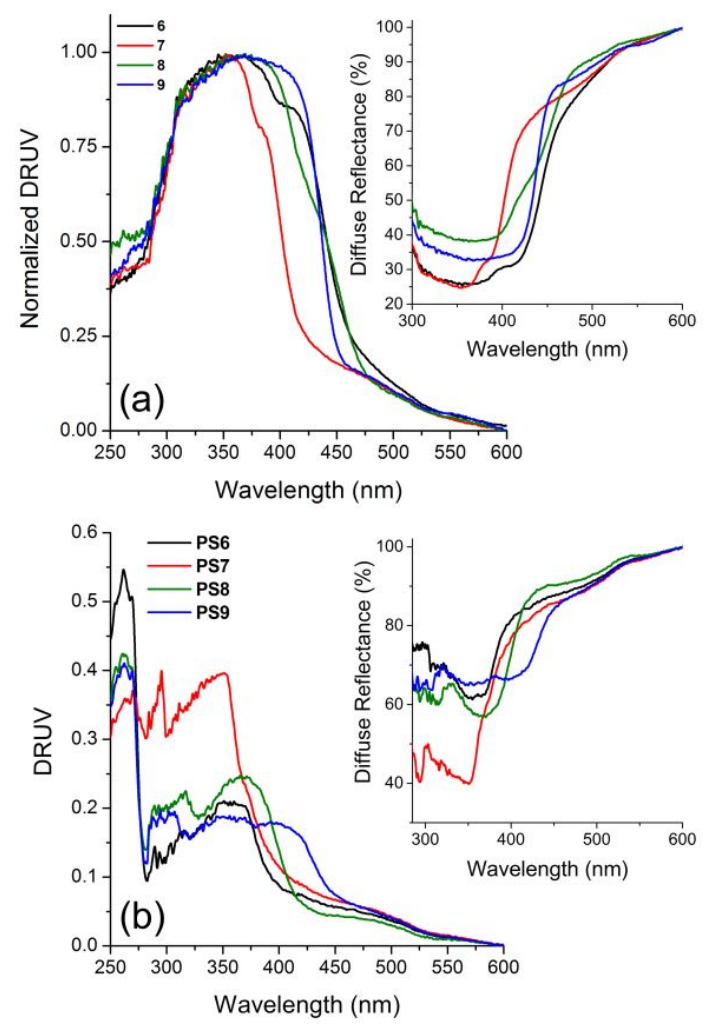

Figure S39. Normalized UV-Vis absorption (DRUV) spectra of (a) ATRP initiators 6-9 and (b) respective polymers PS6-9 in the solid state. The respective insets present the diffuse reflectance spectra of each set of samples. 

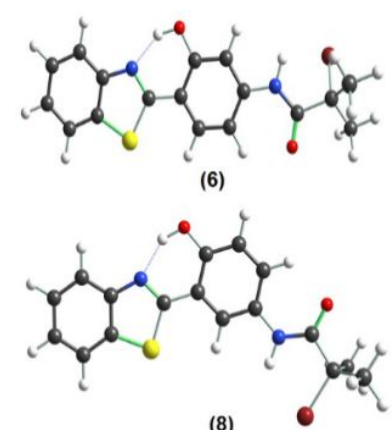

(8)
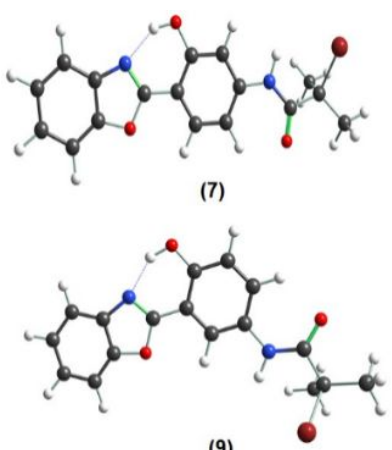

(9)

Figure S40. Optimized geometries in the ground state for the initiators 6-9 in THF, the calculations were performed using CAM-B3LYP/cc-pVDZ for the optimization. 


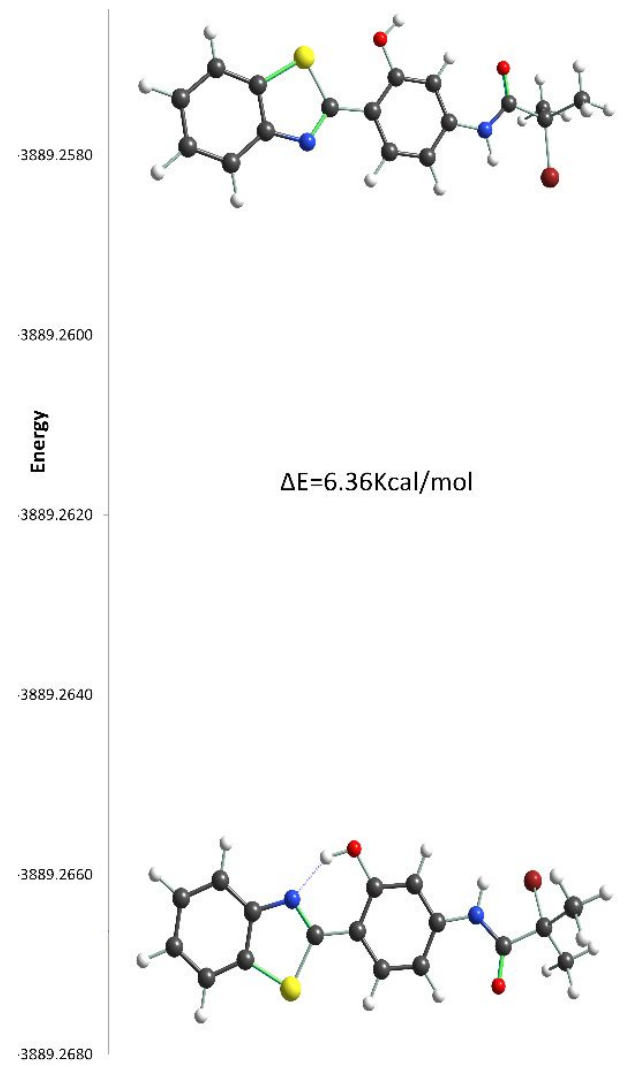

Figure S41. Energy diagram for the rotation of conformer enol cis of initiator 6 to the conformer enol trans calculated at jun-cc-pVTZ level. 


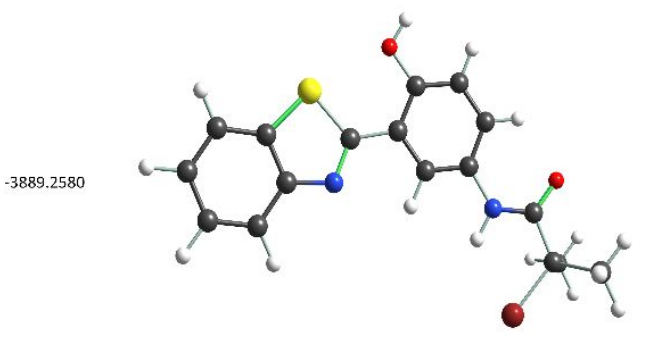

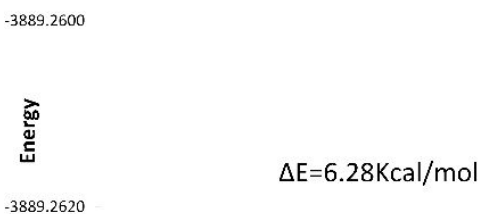

$-3889.2640$

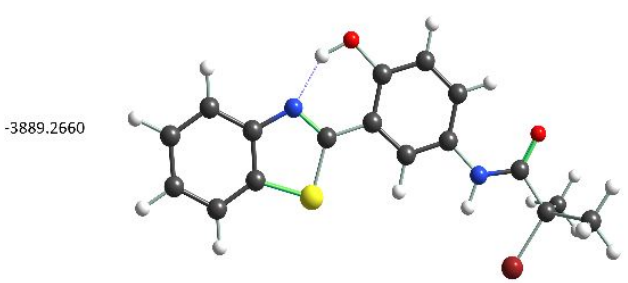

$-3889.2680$

Figure S42. Energy diagram for the rotation of conformer enol cis of initiator 8 to the conformer enol trans calculated at jun-cc-pVTZ level. 

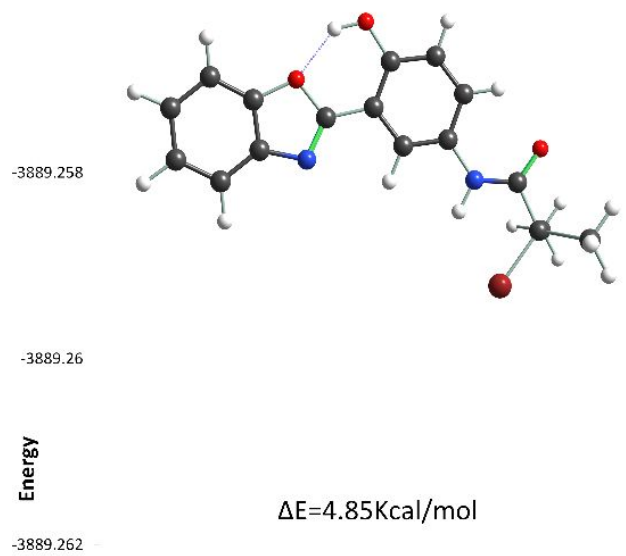

$-3889.264$

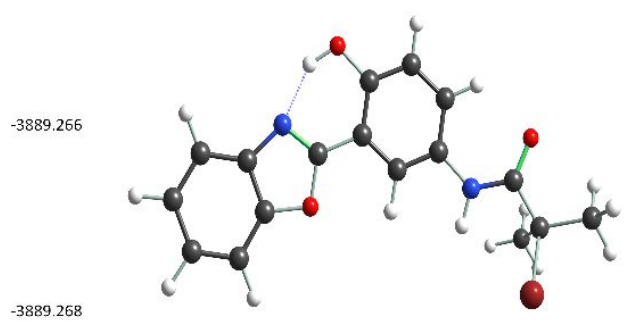

Figure S43. Energy diagram for the rotation of conformer enol cis of initiator 9 to the conformer enol trans calculated at jun-cc-pVTZ level. 


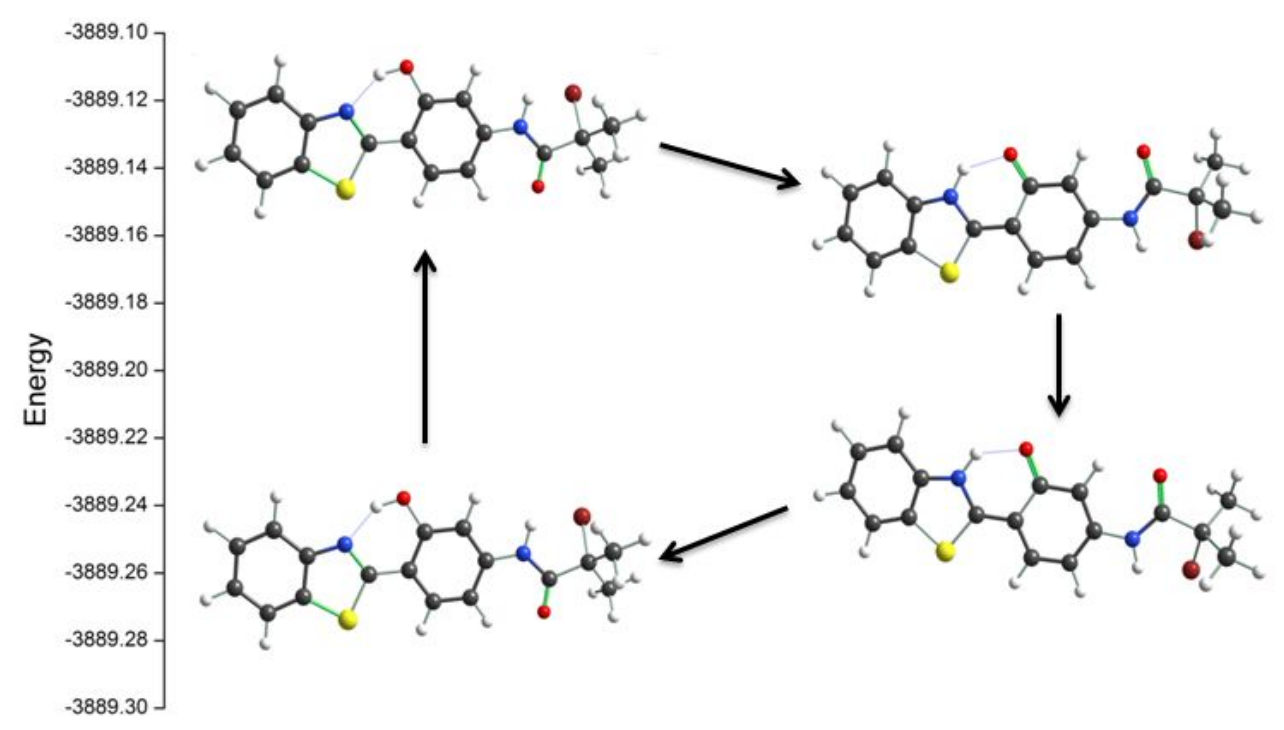

Figure S44. Energy diagram for initiator 6, calculated at CAM-B3LYP/junccpVTZ level. The energies are in atomic units.

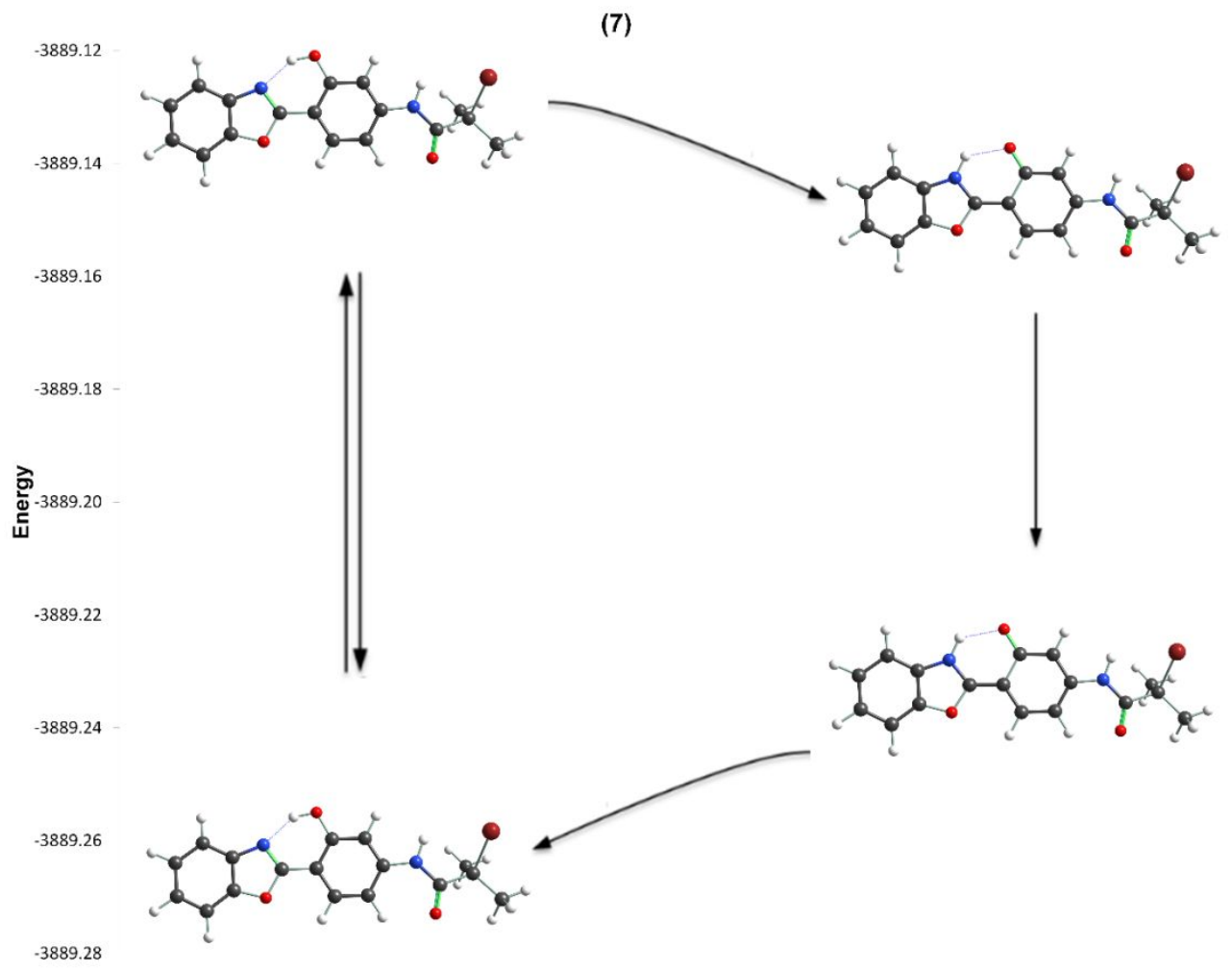

Figure S45. Energy diagram for initiator 7, calculated at CAM-B3LYP/junccpVTZ level. The energies are in atomic units. 


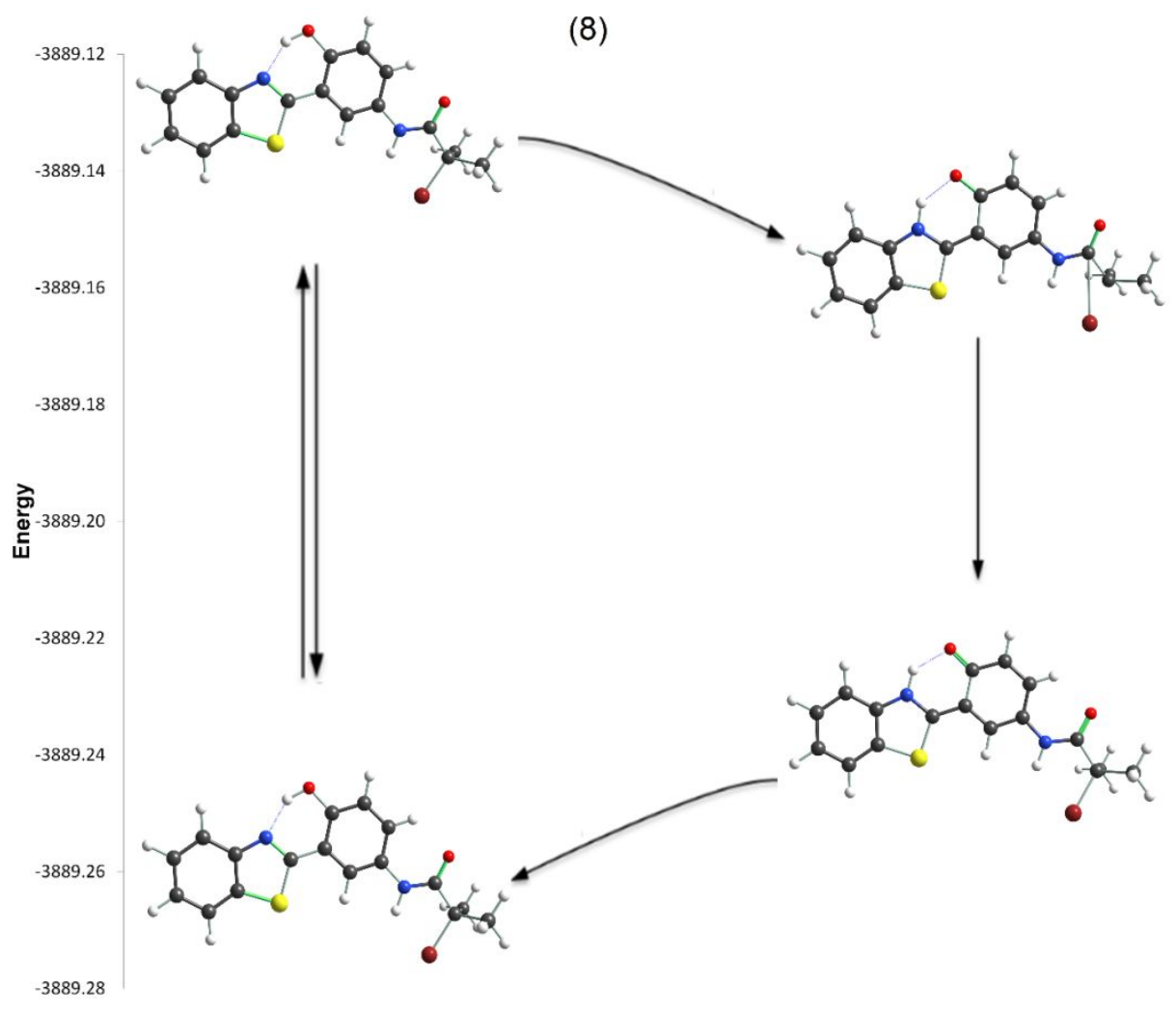

Figure S46. Energy diagram for initiator 8, calculated at CAM-B3LYP/junccpVTZ level. The energies are in atomic units.

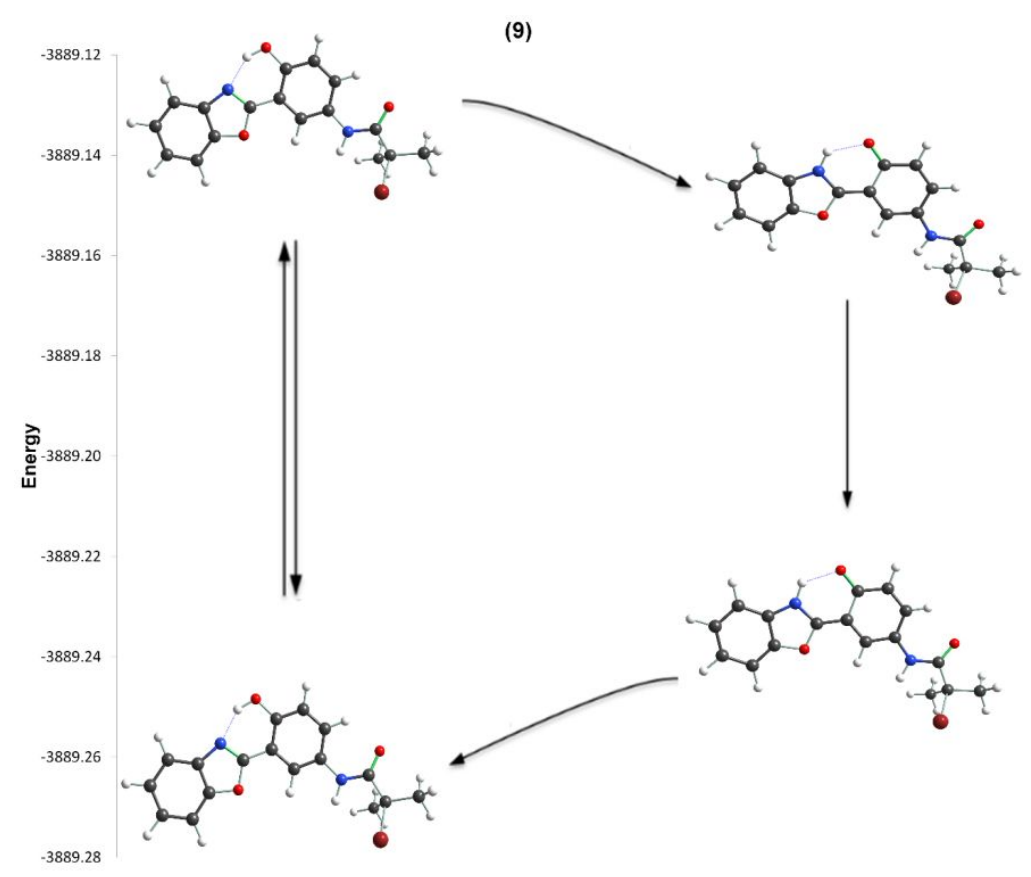

Figure S47. Energy diagram for initiator 9, calculated at CAM-B3LYP/junccpVTZ level. The energies are in atomic units. 
Table S3o. Theoretical results for absorption $\left(\lambda_{\text {abs }}\right)$ and emission $\left(\lambda_{\text {em }}\right)$ maxima $(\mathrm{nm})$ and Stokes shift $\left(\Delta \lambda_{\mathrm{ST}}\right)\left(\mathrm{nm} / \mathrm{cm}^{-1}\right)$.

\begin{tabular}{cccccc}
\hline \multirow{2}{*}{ Sample } & $\lambda_{\text {abs }}$ & \multicolumn{2}{c}{ Enol $^{*}\left(\mathbf{E}^{*}\right)$} & \multicolumn{2}{c}{ Keto $^{*}\left(\mathbf{K}^{*}\right)$} \\
\cline { 3 - 6 } & 327.82 & 389.29 & $61 / 482$ & 431.1 & $103 / 9709$ \\
\hline $\mathbf{6}$ & 312.97 & 370.02 & $57 / 493$ & 413.64 & $101 / 9901$ \\
$\mathbf{7}$ & 334.58 & 403.08 & $69 / 508$ & 491.67 & $157 / 6369$ \\
$\mathbf{8}$ & 322.11 & 379.04 & $57 / 466$ & 470.1 & $148 / 6757$ \\
$\mathbf{9}$ & & & & & \\
\hline
\end{tabular}

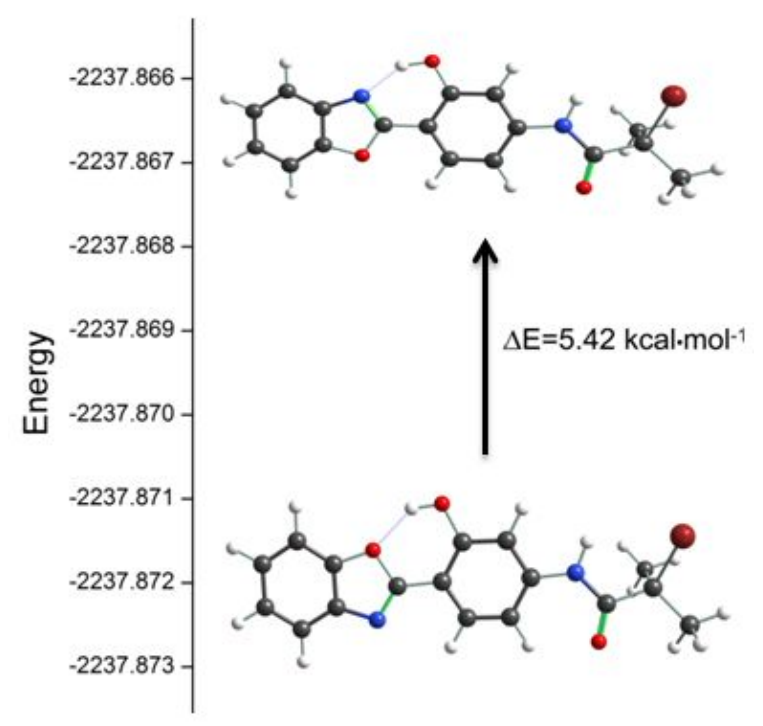

Figure S48. Energy diagram for the rotation of conformer enol cis of initiator 7 to the conformer enol trans calculated at jun-cc-pVTZ level. 